\title{
General relativistic dynamics of compact binaries at the third post-Newtonian order
}

\author{
Luc Blanchet \\ Département d'Astrophysique Relativiste et de Cosmologie, \\ Centre National de la Recherche Scientifique (UMR 8629), \\ Observatoire de Paris, 92195 Meudon Cedex, France \\ Guillaume Faye \\ Département d'Astrophysique Relativiste et de Cosmologie, \\ Centre National de la Recherche Scientifique (UMR 8629), \\ Observatoire de Paris, 92195 Meudon Cedex, France
}

(October 24, 2018)

\begin{abstract}
The general relativistic corrections in the equations of motion and associated energy of a binary system of point-like masses are derived at the third post-Newtonian $(3 \mathrm{PN})$ order. The derivation is based on a post-Newtonian expansion of the metric in harmonic coordinates at the 3PN approximation. The metric is parametrized by appropriate non-linear potentials, which are evaluated in the case of two point-particles using a Lorentzian version of an Hadamard regularization which has been defined in previous works. Distributional forms and distributional derivatives constructed from this regularization are employed systematically. The equations of motion of the particles are geodesic-like with respect to the regularized metric. Crucial contributions to the acceleration are associated with the non-distributivity of the Hadamard regularization and the violation of the Leibniz rule by the distributional derivative. The final equations of motion at the 3PN order are invariant under global Lorentz transformations, and admit a conserved energy (neglecting the radiation reaction force at the $2.5 \mathrm{PN}$ order). However, they are not fully determined, as they depend on one arbitrary constant, which reflects probably a physical incompleteness of the point-mass regularization. The results of this paper should be useful when comparing theory to the observations of gravitational waves from binary systems in future detectors VIRGO and LIGO.
\end{abstract}

Typeset using REVTEX 


\section{INTRODUCTION}

The present work is a contribution to the problem of the dynamics of two compact objects at the so-called third post-Newtonian $(3 \mathrm{PN})$ approximation of general relativity. By $3 \mathrm{PN}$ we mean the relativistic corrections in the binary's equations of motion corresponding to the order $1 / c^{6}$ relatively to the Newtonian acceleration, when the speed of light $c$ tends to infinity. Why studying the equations of motion to such a frightful post-Newtonian order? A side reason is the strange beauty of the post-Newtonian expansion, which becomes quite intricate at the $3 \mathrm{PN}$ order, where it requires some interesting mathematical methods. The main reason, however, is that inspiralling compact binaries, namely systems of two neutron stars or black holes (or one of each) moving on a relativistic orbit prior to their final merger, should be routinely observed by the gravitational-wave detectors LIGO, VIRGO and their fellows. Several analysis show that the post-Newtonian templates required for the detection and parameter extraction of inspiralling compact binaries should include the relativistic corrections in the binary's orbital phase at approximately the level of the 3PN order [1 1 ].

Lorentz and Droste [8] were the first to obtain the correct equations of motion of two non-spinning particles at the 1PN approximation (see [9,10] for reviews). An important work by Einstein, Infeld and Hoffmann [11]13] showed that the 1PN acceleration can in fact be deduced from the vacuum gravitational field outside the masses. This result is interesting because, in their approach, the bodies are allowed to carry a strong internal gravity. Unfortunately, the computation of the surface integrals surrounding the masses is very difficult even at the $1 \mathrm{PN}$ order (see [14 for a recent derivation of the Einstein-Infeld-Hoffmann equations). The same equations were also obtained by Fock and followers [15 [17] for the motion of the centers of mass of bodies with finite size. The next approximation, 2PN, has been tackled by Otha, Okamura, Kimura and Hiida [18 20] with a direct post-Newtonian computation of the Hamiltonian of $N$ point-particles; however the first complete 2-particle case in their framework is only given by Damour and Schäfer [21, and the fully explicit 3-particle case is due to Schäfer [22]. Up to the $2 \mathrm{PN}$ level, the equations of motion are conservative (existence of ten conserved quantities, including a conserved energy). The non-conservative effect, which is associated to the radiation reaction force, arises at the $2.5 \mathrm{PN}$ order. The first correct equations of motion of two masses at the 2.5PN order were obtained by Damour, Deruelle and collaborators [23 26] in harmonic coordinates. These equations are applicable

to systems of strongly self-gravitating bodies such as neutron stars (see Damour [9,10] for the proof). Moreover, Kopejkin [27] and Grishchuk and Kopejkin 28] obtained the same equations in the case of weakly self-gravitating extended bodies. The corresponding result at 2.5PN order was also derived by Schäfer [29,30] using the ADM Hamiltonian approach. Later, the harmonic-coordinates equations of motion were re-computed by Blanchet, Faye and Ponsot 31 following a direct post-Newtonian iteration of the field equations. Some of the latter derivations [23,29,31] opt for a formal description of the compact objects by point-particles. There is a nice agreement between all these different methods at the $2.5 \mathrm{PN}$ order. In addition, the complete $2.5 \mathrm{PN}$ gravitational field generated by point-particles in 
harmonic coordinates was derived in 31 .

At the 3PN order, the equations of motion have been obtained using a Hamiltonian and formal delta functions by Jaranowski and Schäfer [32,33] in the center-of-mass frame, and by Damour, Jaranowski and Schäfer [35] in an arbitrary frame. These authors found an irreducible ambiguity linked probably with an incompleteness in the regularization of the infinite self-field of the particles. In this paper, following the method initiated in [31], we address the problem of the 3PN dynamics of point-particles in harmonic coordinates. Earlier in [34], our result has been already discussed and reported in the case of circular orbits. We find the presence of one (and only one) undetermined coefficient in the 3PN equations of motion, in agreement with [32,33,35. Recently, the physical equivalence between our result in harmonic coordinates and the result given by the ADM-Hamiltonian approach has been established 36, 37.

Another line of research, initiated by Chandrasekhar and collaborators [38, 39], consists of working with continuous hydrodynamical fluids from the start, and derived the metric and equations of motion of an isolated fluid ball up to the 2.5PN order 40 43] (the derivation in the case of two fluid balls, in the limit of zero size of the bodies, being due to [27,28]). Our iteration of the gravitational field and equations of motion in the previous paper [31] is close to the latter line of work in the sense that it is based on the reduction of some general expressions of the post-Newtonian metric, initially valid for continuous fluids, to point-like particles. The choice of point-particles, adopted here as well, is motivated by the efficiency of the delta-functions in performing some complicated non-linear integrations. The price we have to pay is the necessity of a self-field regularization. We apply systematically in this paper the regularization of Hadamard, based on the concept of "partie finie" of singular functions and divergent integrals [44 46]. This technique is indeed extensively used in this field [23,29, 31, 32, More precisely, we apply a variant of the Hadamard regularization, together with a theory of pseudo-functions and distributional derivatives, that is compatible with the Lorentzian structure of the gravitational field. All the details about this regularization can be found in [47,48]. We use notably a specific form of distributional stress-energy tensor based on "delta-pseudo-functions" (with support limited to the world lines of the particles). In a sense, these delta-pseudo-functions constitute some mathematically well-defined versions of the so-called "good delta functions" introduced long ago by Infeld 49 (see also an appendix in the book of Infeld and Plebanski [50]).

Thus, we are using a formal regularization method, based on a clear mathematical framework 47,48, but that we cannot justify physically (why should the compact objects be described by such delta-pseudo-function singularities?). Definitely, our main justification is that this method permits the derivation of a result in a consistent and well-defined way (i.e. all the difficult non-linear integrals at the 3PN order are computed unambiguously). Furthermore, we shall check that some different regularization prescriptions yield equations of motion that are physically the same, in the sense that they differ from each other by merely a coordinate transformation. Moreover, a justification a posteriori is that the end result owns all the physical properties that we expect the true equations of motion of compact 
objects to obey. In particular, there is agreement with the known results at the previous post-Newtonian orders, we get the correct geodesic limit for the motion of a test particle in a Schwarzschild background, find that the 3PN equations of motion stay invariant under global Lorentz transformations, and obtain a conserved energy at 3PN (neglecting the radiation reaction). The investigation of the Lagrangian formulation of the equations is dealt with in a separate work 37.

Ideally, one should perform, instead of a computation valid only for point particles (and necessitating a regularization), a complete calculation in the case of extended bodies, i.e. taking into account the details of the internal structure of the bodies. By considering the limit where the radius of the two objects tend to zero, one should recover the same result as obtained by means of the point-mass regularization. This would demonstrate the suitability of the regularization. In fact, this program has been achieved at the $2 \mathrm{PN}$ order by Grishchuk and Kopejkin [27,28], who proved that the compactness parameters associated with each object disappear from the equations of motion, and obtained the same equations as in the case of point particles. At the $3 \mathrm{PN}$ order there is no such a proof that the method with extended bodies would give the same result as with point particles.

The main problem is that from the $3 \mathrm{PN}$ level one cannot compute the most difficult of the non-linear integrals in closed form for two extended fluid bodies of finite radius (though these integrals could perhaps be obtained as power series valid when the two radius tend to zero). Presently the only approach which is able to overcome this problem is the one followed in this paper: namely, to model the source by delta functions and to use a regularization. The price we have to pay is the appearance of one physical undetermined coefficient at the $3 \mathrm{PN}$ order. As a consequence, this method should be completed (hopefully in a future work) by the study of the limit relation of the point-particle result with the physical result valid for extended bodies in the limit of zero size. This study should probably give the value of the undetermined parameter left out by the regularization.

The plan of this paper is the following. In Section II, we review some necessary tools concerning the regularization and the definition of the point-particle model. In Section III, we perform the post-Newtonian iteration of the field equations and write the 3PN metric in terms of some convenient non-linear potentials. Section $\mathbb{\mathbb { V }}$ is devoted to the computation of the compact-support and quadratically non-linear parts of the potentials. The most difficult

potentials, involving notably some non-compact cubic non-linearities at $1 \mathrm{PN}$, are obtained in Section $\mathrm{V}$. The so-called Leibniz and non-distributivity contributions to the equations of motion are derived in Section VI. Finally, we present in Section VII the result for the compact binary's 3PN acceleration (in the case of general orbits) and the associated 3PN energy.

\section{HADAMARD REGULARIZATION}

In this section we present a short account about the regularization of Hadamard 44,45, the associated generalized or pseudo-functions, and the choice of stress-energy tensor for 
point-particles. We follow (and refer to) the detailed investigations in [47,48]. Consider the class $\mathcal{F}$ of functions $F(\mathbf{x})$ which are smooth $\left(C^{\infty}\right)$ on $\mathbb{R}^{3}$ deprived from two singular points $\mathbf{y}_{1}$ and $\mathbf{y}_{2}$, around which they admit a power-like singular expansion of the type

$$
\forall n \in \mathbb{N}, \quad F(\mathbf{x})=\sum_{a_{0} \leq a \leq n} r_{1}^{a} f_{a}\left(\mathbf{n}_{1}\right)+o\left(r_{1}^{n}\right),
$$

and similarly for the other point 2. Here $r_{1}=\left|\mathbf{x}-\mathbf{y}_{1}\right| \rightarrow 0$, and the coefficients ${ }_{1} f_{a}$ of the various powers of $r_{1}$ depend on the unit direction $\mathbf{n}_{1}=\left(\mathbf{x}-\mathbf{y}_{1}\right) / r_{1}$ of approach to the singular point. The powers $a$ of $r_{1}$ are real, range in discrete steps (i.e. $a \in\left(a_{i}\right)_{i \in \mathbb{N}}$ ) and are bounded from below $\left(a_{0} \leq a\right)$. The coefficients ${ }_{1} f_{a}$ (and ${ }_{2} f_{a}$ ) for which $a<0$ are referred to as the singular coefficients of $F$. If $F$ and $G$ belong to $\mathcal{F}$ so does the ordinary pointwise product $F G$, as well as the ordinary gradient $\partial_{i} F$. We define the Hadamard "partie finie" of $F$ at the location of the singular point 1 as

$$
(F)_{1}=\int \frac{d \Omega_{1}}{4 \pi} f_{0}\left(\mathbf{n}_{1}\right),
$$

where $d \Omega_{1}=d \Omega\left(\mathbf{n}_{1}\right)$ denotes the solid angle element centered on $\mathbf{y}_{1}$ and of direction $\mathbf{n}_{1}$. Furthermore, the Hadamard partie finie (Pf) of the integral $\int d^{3} \mathbf{x} F$, which is in general divergent at the two singular points $\mathbf{y}_{1}$ and $\mathbf{y}_{2}$ (we assume no divergence at infinity), is defined by

$$
\begin{aligned}
\mathrm{Pf}_{s_{1}, s_{2}} \int d^{3} \mathbf{x} F=\lim _{s \rightarrow 0} & \left\{\int_{\mathcal{D}(s)} d^{3} \mathbf{x} F\right. \\
+ & \left.4 \pi \sum_{a+3<0} \frac{s^{a+3}}{a+3}\left(\frac{F}{r_{1}^{a}}\right)_{1}+4 \pi \ln \left(\frac{s}{s_{1}}\right)\left(r_{1}^{3} F\right)_{1}+1 \leftrightarrow 2\right\} .
\end{aligned}
$$

The first term integrates over a domain $\mathcal{D}(s)$ defined as $\mathbb{R}^{3}$ to which the two spherical balls $r_{1} \leq s$ and $r_{2} \leq s$ of radius $s$ and centered on the singularities are removed. The other terms, in which the value of a function at 1 takes the meaning (2.2), are such that they cancel out the divergent part of the first term in the limit where $s \rightarrow 0$ (the symbol $1 \leftrightarrow 2$ means the same terms but corresponding to the other point 2). Note that the Hadamard partie finie depends on two strictly positive constants $s_{1}$ and $s_{2}$, associated with the logarithms in (2.3). See [47] and Section $\nabla$ below for alternative expressions of the partie-finie integral.

To any $F \in \mathcal{F}$ we associate a partie finie pseudo-function $\operatorname{Pf} F$ defined as the linear form on $\mathcal{F}$ given by the duality bracket,

$$
\forall G \in \mathcal{F}, \quad<\operatorname{Pf} F, G>=\operatorname{Pf} \int d^{3} \mathbf{x} F G .
$$

The pseudo-function $\operatorname{Pf} F$, when restricted to the set of smooth functions with compact support, is a distribution in the sense of Schwartz [45]. The product of pseudo-functions 
coincides with the ordinary pointwise product, namely $\operatorname{Pf} F \cdot \operatorname{Pf} G=\operatorname{Pf}(F G)$. A particularly interesting pseudo-function, constructed in [47] on the basis of the Riesz delta function [51], is the delta-pseudo-function $\operatorname{Pf} \delta_{1}$, which plays the same role as the Dirac measure in distribution theory, in the sense that

$$
\forall F \in \mathcal{F}, \quad<\operatorname{Pf} \delta_{1}, F>=\operatorname{Pf} \int d^{3} \mathbf{x} \delta_{1} F=(F)_{1}
$$

where $(F)_{1}$ is the partie finie of $F$ as defined by (2.2). From the product of $\operatorname{Pf} \delta_{1}$ with any $\operatorname{Pf} F$ we obtain the new pseudo-function $\operatorname{Pf}\left(F \delta_{1}\right)$ which is such that

$$
\forall G \in \mathcal{F}, \quad<\operatorname{Pf}\left(F \delta_{1}\right), G>=(F G)_{1}
$$

Next, the spatial derivative of a pseudo-function of the type $\operatorname{Pf} F$, namely $\partial_{i}(\operatorname{Pf} F)$, is treated as follows. Essentially, we require in [47 the so-called rule of integration by parts, namely that we are allowed to freely operate by parts any duality bracket, with the allintegrated ("surface") terms always zero exactly like in the case of non-singular functions. This requirement is motivated by our will that a computation involving singular functions be as much as possible the same as a computation valid for regular functions. Thus,

$$
\forall F, G \in \mathcal{F}, \quad<\partial_{i}(\operatorname{Pf} F), G>=-<\partial_{i}(\operatorname{Pf} G), F>
$$

Furthermore, we assume that when all the singular coefficients of $F$ vanish, the derivative of $\operatorname{Pf} F$ reduces to the ordinary derivative, i.e. $\partial_{i}(\operatorname{Pf} F)=\operatorname{Pf}\left(\partial_{i} F\right)$. As a particular case, we see from these assumptions that the integral of a gradient is always zero: $\left\langle\partial_{i}(\operatorname{Pf} F), 1\right\rangle=0$. Certainly this should be the case if we want to apply to the case of singular sources a formula which is defined modulo a total divergence for continuous sources. We have also at our disposal a distributional time derivative and the associated partial derivatives with respect to the points 1 and 2 (see Section IX in [47). The difference between the distributional derivative and the ordinary one gives the distributional terms $\mathrm{D}_{i}[F]$ present in the derivative of $F$,

$$
\partial_{i}(\operatorname{Pf} F)=\operatorname{Pf}\left(\partial_{i} F\right)+\mathrm{D}_{i}[F]
$$

A simple solution of our basic relation (2.7), denoted $\mathrm{D}_{i}^{\text {part }}[F]$ standing for the "particular" solution, was obtained in [47] as the following functional of the singular coefficients of $F$,

$$
\mathrm{D}_{i}^{\text {part }}[F]=4 \pi \operatorname{Pf}\left(n_{1}^{i}\left[\frac{1}{2} r_{1} f_{-1}+\sum_{k \geq 0} \frac{1}{r_{1}^{k}} f_{-2-k}\right] \delta_{1}\right)+1 \leftrightarrow 2
$$


where we assume for simplicity that the powers $a$ in the expansion of $F$ are relative integers, $a \in \mathbb{Z}$. (The sum over $k$ is always finite.) The distributional term (2.9) is of the form $\operatorname{Pf}\left(G \delta_{1}\right)$ (plus $1 \leftrightarrow 2$ ). However, the particular solution (2.9) does not represent the most satisfying derivative operator acting on pseudo-functions. It is shown in [47 that one can require also the rule of commutation of successive derivatives, which is not satisfied in general by (2.9). Still we are motivated when asking for the commutation of derivatives that the properties of our distributional derivative be the closest possible to those of the ordinary derivative. The most general derivative operator satisfying the same properties as (2.9) and, in addition, the commutation of derivatives (Schwarz lemma) is given by

$$
\mathrm{D}_{i}[F]=4 \pi \sum_{l=0}^{+\infty} \operatorname{Pf}\left(C_{l}\left[\begin{array}{c}
n_{1}^{i L} \hat{f}_{-1}^{L}-n_{1}^{L} \hat{f}_{-1}^{i L} \\
1
\end{array}\right] r_{1} \delta_{1}+\sum_{k \geq 0} \frac{n_{1}^{i L}}{r_{1}^{k}} \hat{f}_{-2-k}^{L} \delta_{1}\right)+1 \leftrightarrow 2
$$

where we denote by ${ }_{1} \hat{f}_{a}^{L}$ the STF-harmonics of the expansion coefficient ${ }_{1} f_{a}$, which is such that ${ }_{1} f_{a}=\sum_{l \geq 0} n_{1}^{L} \hat{f}_{a}^{L}$ (see 477 for details). A particularity of this derivative is that it depends on an arbitrary constant $K$ through the $l$-dependent coefficient

$$
C_{l}=(l+1)\left[K+\sum_{j=1}^{l} \frac{1}{j+1}\right]
$$

Both the derivative operators (2.9) and (2.10)-(2.11) represent some generalizations of the Schwartz distributional derivative [45], that are appropriate to the singular functions of the class $\mathcal{F}$. In was shown in Section VIII in [47 that the distributional terms associated with the $l$ th distributional derivative, i.e. $\mathrm{D}_{L}[F]=\partial_{L} \operatorname{Pf} F-\operatorname{Pf} \partial_{L} F$, where $L=i_{1} i_{2} \ldots i_{l}$ denotes a multi-index composed of $l$ indices, is given by

$$
\mathrm{D}_{L}[F]=\sum_{k=1}^{l} \partial_{i_{1} \ldots i_{k-1}} \mathrm{D}_{i_{k}}\left[\partial_{i_{k+1} \ldots i_{l}} F\right]
$$

Though this is not manifest on this formula, $\mathrm{D}_{L}[F]$ in the case of the "correct" derivative (2.10)-(2.11) is fully symmetric in the $l$ indices forming $L$. Note that neither of the derivatives (2.9) and (2.10) satisfy the Leibniz rule for the derivation of a product. Rather, the investigation in [47 has suggested that, in order to construct a consistent theory (using the "ordinary" product for pseudo-functions), the Leibniz rule should in a sense be weakened, and replaced by the rule of integration by part (2.7), which is in fact nothing but an "integrated" version of the Leibniz rule. In this paper, we shall be careful about taking into account the violation of the Leibniz rule by the distributional derivative. We shall also investigate the fate of the constant $K$ appearing in (2.11) when deriving the 3PN equations of motion.

The Hadamard regularization $(F)_{1}$ is defined by $(2.2)$ in a preferred spatial hypersurface $t=$ const of a coordinate system, and consequently is not a priori compatible with the global 
Lorentz invariance of special relativity. If we restrict the coordinates to satisfy the usual harmonic gauge conditions, we introduce a preferred Minkowski metric, and thus we can view the gravitational field as a relativistic Lorentz tensor field in special relativity, that we certainly want to regularize in a Lorentz-invariant way. To achieve this we defined in [48] a new regularization, denoted $[F]_{1}$, by performing the Hadamard regularization within the spatial hypersurface which is geometrically orthogonal (in the Minkowskian sense) to the four-velocity of the particle. In a sense, the regularization $[F]_{1}$ permits us to get rid of the anisotropic Lorentz contraction due to their motion when defining the point-masses. The Lorentzian regularization $[F]_{1}$ differs from the old one $(F)_{1}$ by relativistic corrections of order $1 / c^{2}$ at least. All the formulas for its computation are given in 48 in the form of some infinite expansion series in the relativistic parameter $1 / c^{2}$. The regularization $[F]_{1}$ plays a crucial role in the present computation, as it will be seen that the breakdown of the Lorentz invariance due to the old regularization $(F)_{1}$ occurs precisely at the $3 \mathrm{PN}$ order in the equations of motion. Associated with the new regularization in 48] we can define, exactly like in (2.5), a "Lorentzian" delta-pseudo-function $\operatorname{Pf} \Delta_{1}$ which when applied on any $F$ gives $[F]_{1}$. More generally we have, similarly to (2.6),

$$
\forall G \in \mathcal{F}, \quad<\operatorname{Pf}\left(F \Delta_{1}\right), G>=[F G]_{1} .
$$

Notice that as a general rule we are not allowed to replace $F$ in the pseudo-function $\operatorname{Pf}\left(F \Delta_{1}\right)$ by its regularized value, i.e. $\operatorname{Pf}\left(F \Delta_{1}\right) \neq[F]_{1} \operatorname{Pf} \Delta_{1}$. This is a consequence of the "nondistributivity" of the Hadamard partie finie with respect to the multiplication, i.e. $[F G]_{1} \neq$ $[F]_{1}[G]_{1}$. In this paper, we shall (heuristically) model the compact objects by point-particles, and in order to describe those point-particles we shall use a particular representation of the stress-energy tensor which has been derived in Section $\mathrm{V}$ of [48] on the basis of an action principle compatible with the Lorentzian regularization $[F]_{1}$. The proposal made in 48 is that

$$
T^{\mu \nu}=m_{1} c \frac{v_{1}^{\mu} v_{1}^{\nu}}{\sqrt{-\left[g_{\rho \sigma}\right]_{1} v_{1}^{\rho} v_{1}^{\sigma}}} \operatorname{Pf}\left(\frac{\Delta_{1}}{\sqrt{-g}}\right)+1 \leftrightarrow 2 .
$$

Most importantly about this expression are the facts that (i) $\left[g_{\rho \sigma}\right]_{1}$ within the first factor means the Lorentzian regularization of the metric in the previous sense, (ii) the pseudofunction $\operatorname{Pf}\left(\frac{1}{\sqrt{-g}} \Delta_{1}\right)$ is of the type $\operatorname{Pf}\left(F \Delta_{1}\right)$ which is defined by (2.13). We denote by $m_{1}$ the (constant) mass of the particle 1 , by $\mathbf{y}_{1}(t)$ its trajectory parametrized by the harmoniccoordinate time $t$, and by $\mathbf{v}_{1}(t)=d \mathbf{y}_{1} / d t$ the coordinate velocity [with $v_{1}^{\mu}=\left(c, \mathbf{v}_{1}\right)$ ]. In the next section, we look for solutions in the form of post-Newtonian expansions of the Einstein field equations having the latter stress-energy tensor as a matter source.

\section{THE THIRD POST-NEWTONIAN METRIC}




\section{A. The Einstein field equations}

We base our investigation on a system of harmonic coordinates $x^{0}=c t,\left(x^{i}\right)=\mathbf{x}$, since such coordinates are especially well-suited to a post-Newtonian (or post-Minkowskian) iteration of the field equations. We define the gravitational perturbation $h^{\mu \nu}$ associated with the "gothic metric" as

$$
h^{\mu \nu}=\sqrt{-g} g^{\mu \nu}-\eta^{\mu \nu}
$$

with $g^{\mu \nu}$ and $g$ being the inverse and the determinant of the covariant metric $g_{\mu \nu}$, and where $\eta^{\mu \nu}=\operatorname{diag}(-1,1,1,1)$ denotes an auxiliary Minkowski metric. Under the condition of harmonic coordinates,

$$
\partial_{\nu} h^{\mu \nu}=0
$$

the Einstein field equations take the form

$$
\square h^{\mu \nu}=\frac{16 \pi G}{c^{4}}|g| T^{\mu \nu}+\Lambda^{\mu \nu},
$$

where $\square=\eta^{\mu \nu} \partial_{\mu} \partial_{\nu}$ denotes the flat d'Alembertian operator, where $T^{\mu \nu}$ is the matter stress-

energy tensor defined in our case of point-particle binaries by (2.14), and where $\Lambda^{\mu \nu}$ is the gravitational source term. Using the integral of the retarded potentials given by

$$
\square_{\mathcal{R}}^{-1} \tau(\mathbf{x}, t)=\int \frac{d^{3} \mathbf{x}^{\prime}}{-4 \pi} \frac{\tau\left(\mathbf{x}^{\prime}, t-\left|\mathbf{x}-\mathbf{x}^{\prime}\right| / c\right)}{\left|\mathbf{x}-\mathbf{x}^{\prime}\right|},
$$

we can also re-write the solution of the field equations (3.3), under a condition of no-incoming radiation, under the form

$$
h^{\mu \nu}=\square_{\mathcal{R}}^{-1}\left[\frac{16 \pi G}{c^{4}}|g| T^{\mu \nu}+\Lambda^{\mu \nu}\right] .
$$

The gravitational source term $\Lambda^{\mu \nu}$ is related to the Landau-Lifchitz pseudo-tensor $t_{\mathrm{LL}}^{\mu \nu}$ by

$$
\Lambda^{\mu \nu}=\frac{16 \pi G}{c^{4}}|g| t_{\mathrm{LL}}^{\mu \nu}+\partial_{\rho} h^{\mu \sigma} \partial_{\sigma} h^{\nu \rho}-h^{\rho \sigma} \partial_{\rho \sigma} h^{\mu \nu}
$$

and can be expanded as an infinite non-linear series in $h$ and its first and second space-time derivatives; in this paper we need only the non-linear terms up to the quartic $\left(h^{4}\right.$ or $\left.G^{4}\right)$ level, viz 


$$
\Lambda^{\mu \nu}=N^{\mu \nu}(h, h)+M^{\mu \nu}(h, h, h)+L^{\mu \nu}(h, h, h, h)+\mathcal{O}\left(h^{5}\right)
$$

where the quadratic non-linearity $N^{\mu \nu}$, the cubic one $M^{\mu \nu}$ and the quartic $L^{\mu \nu}$ are explicitly given by

$$
\begin{aligned}
& N^{\mu \nu}=-h^{\rho \sigma} \partial_{\rho \sigma} h^{\mu \nu}+\frac{1}{2} \partial^{\mu} h_{\rho \sigma} \partial^{\nu} h^{\rho \sigma}-\frac{1}{4} \partial^{\mu} h \partial^{\nu} h+\partial_{\sigma} h^{\mu \rho}\left(\partial^{\sigma} h_{\rho}^{\nu}+\partial_{\rho} h^{\nu \sigma}\right) \\
& -2 \partial^{(\mu} h_{\rho \sigma} \partial^{\rho} h^{\nu) \sigma}+\eta^{\mu \nu}\left[-\frac{1}{4} \partial_{\tau} h_{\rho \sigma} \partial^{\tau} h^{\rho \sigma}+\frac{1}{8} \partial_{\rho} h \partial^{\rho} h+\frac{1}{2} \partial_{\rho} h_{\sigma \tau} \partial^{\sigma} h^{\rho \tau}\right], \\
& M^{\mu \nu}=-h^{\rho \sigma}\left(\partial^{\mu} h_{\rho \tau} \partial^{\nu} h_{\sigma}^{\tau}+\partial_{\tau} h_{\rho}^{\mu} \partial^{\tau} h_{\sigma}^{\nu}-\partial_{\rho} h_{\tau}^{\mu} \partial_{\sigma} h^{\nu \tau}\right) \\
& +h^{\mu \nu}\left[-\frac{1}{4} \partial_{\tau} h_{\rho \sigma} \partial^{\tau} h^{\rho \sigma}+\frac{1}{8} \partial_{\rho} h \partial^{\rho} h+\frac{1}{2} \partial_{\rho} h_{\sigma \tau} \partial^{\sigma} h^{\rho \tau}\right]+\frac{1}{2} h^{\rho \sigma} \partial^{(\mu} h_{\rho \sigma} \partial^{\nu)} h \\
& +2 h^{\rho \sigma} \partial_{\tau} h_{\rho}^{(\mu} \partial^{\nu)} h_{\sigma}^{\tau}+h^{\rho(\mu}\left[\partial^{\nu)} h_{\sigma \tau} \partial_{\rho} h^{\sigma \tau}-2 \partial_{\sigma} h_{\tau}^{\nu)} \partial_{\rho} h^{\sigma \tau}-\frac{1}{2} \partial^{\nu)} h \partial_{\rho} h\right] \\
& +\eta^{\mu \nu}\left[\frac{1}{8} h^{\rho \sigma} \partial_{\rho} h \partial_{\sigma} h-\frac{1}{4} h^{\rho \sigma} \partial_{\tau} h_{\rho \sigma} \partial^{\tau} h-\frac{1}{4} h^{\tau \lambda} \partial_{\tau} h_{\rho \sigma} \partial_{\lambda} h^{\rho \sigma}\right. \\
& \left.-\frac{1}{2} h^{\tau \lambda} \partial_{\rho} h_{\tau \sigma} \partial^{\sigma} h_{\lambda}^{\rho}+\frac{1}{2} h^{\tau \lambda} \partial_{\rho} h_{\tau}^{\sigma} \partial^{\rho} h_{\lambda \sigma}\right] \text {, } \\
& L^{\mu \nu}=-\frac{1}{2} h^{\mu \nu} h_{\rho \sigma} \partial_{\tau} h^{\rho \lambda} \partial_{\lambda} h^{\sigma \tau}-\frac{1}{4} h^{\mu \nu} h_{\rho \sigma} \partial^{\rho} h_{\tau \lambda} \partial^{\sigma} h^{\tau \lambda}+\frac{1}{8} h^{\mu \nu} h_{\rho \sigma} \partial^{\rho} h \partial^{\sigma} h \\
& +\frac{1}{2} h^{\mu \nu} h_{\rho \sigma} \partial_{\tau} h_{\lambda}^{\rho} \partial^{\tau} h^{\sigma \lambda}-\frac{1}{4} h^{\mu \nu} h_{\rho \sigma} \partial_{\tau} h^{\rho \sigma} \partial^{\tau} h+h_{\rho \lambda} h_{\sigma}^{\lambda} \partial_{\tau} h^{\mu \rho} \partial^{\tau} h^{\nu \sigma} \\
& -2 h_{\rho \lambda} h_{\sigma}^{\lambda} \partial_{\tau} h^{\rho(\mu} \partial^{\nu)} h^{\sigma \tau}+h_{\rho \lambda} h_{\sigma}^{\lambda} \partial^{\mu} h_{\tau}^{\rho} \partial^{\nu} h^{\sigma \tau}-\frac{1}{2} h_{\rho \lambda} h_{\sigma}^{\lambda} \partial^{(\mu} h^{\rho \sigma} \partial^{\nu)} h \\
& -h_{\rho \sigma} h_{\tau \lambda} \partial^{\tau} h^{\mu \rho} \partial^{\lambda} h^{\nu \sigma}+\frac{1}{2} h_{\rho \sigma} h_{\tau \lambda} \partial^{\mu} h^{\rho \tau} \partial^{\nu} h^{\sigma \lambda}-\frac{1}{4} h_{\rho \sigma} h_{\tau \lambda} \partial^{\mu} h^{\rho \sigma} \partial^{\nu} h^{\tau \lambda} \\
& +2 h_{\sigma \tau} h^{\rho(\mu} \partial_{\lambda} h^{\nu) \tau} \partial_{\rho} h^{\sigma \lambda}-2 h_{\sigma \tau} h^{\rho(\mu} \partial^{\nu)} h_{\lambda}^{\sigma} \partial_{\rho} h^{\tau \lambda}+\frac{1}{2} h_{\sigma \tau} h^{\rho(\mu} \partial^{\nu)} h^{\sigma \tau} \partial_{\rho} h \\
& +\frac{1}{2} h_{\sigma \tau} h^{\rho(\mu} \partial^{\nu)} h \partial_{\rho} h^{\sigma \tau}+\frac{1}{2} h^{\mu \rho} h^{\nu \sigma} \partial_{\rho} h_{\tau \lambda} \partial_{\sigma} h^{\tau \lambda}-\frac{1}{4} h^{\mu \rho} h^{\nu \sigma} \partial_{\rho} h \partial_{\sigma} h \\
& +\eta^{\mu \nu}\left[\frac{1}{2} h_{\rho \pi} h_{\sigma}^{\pi} \partial_{\tau} h^{\rho \lambda} \partial_{\lambda} h^{\sigma \tau}-\frac{1}{2} h_{\rho \pi} h_{\sigma}^{\pi} \partial_{\lambda} h_{\tau}^{\rho} \partial^{\lambda} h^{\sigma \tau}+\frac{1}{4} h_{\rho \pi} h_{\sigma}^{\pi} \partial_{\tau} h^{\rho \sigma} \partial^{\tau} h\right. \\
& -\frac{1}{4} h_{\rho \sigma} h_{\tau \lambda} \partial_{\pi} h^{\rho \tau} \partial^{\pi} h^{\sigma \lambda}+\frac{1}{8} h_{\rho \sigma} h_{\tau \lambda} \partial_{\pi} h^{\rho \sigma} \partial^{\pi} h^{\tau \lambda}+\frac{1}{2} h_{\rho \sigma} h_{\tau \lambda} \partial^{\rho} h_{\pi}^{\tau} \partial^{\sigma} h^{\lambda \pi} \\
& \left.-\frac{1}{4} h_{\rho \sigma} h_{\tau \lambda} \partial^{\rho} h^{\tau \lambda} \partial^{\sigma} h\right]
\end{aligned}
$$

All indices are lowered and raised with the Minkowski metric $\eta_{\mu \nu} ; h=\eta^{\mu \nu} h_{\mu \nu}$; the parenthesis around indices indicate the symmetrization.

To describe the matter source we find convenient to introduce the density of mass $\sigma$, of current $\sigma_{i}$ and of stress $\sigma_{i j}$ defined by

$$
\sigma c^{2}=T^{00}+T^{i i},
$$




$$
\begin{aligned}
\sigma_{i} c & =T^{0 i}, \\
\sigma_{i j} & =T^{i j},
\end{aligned}
$$

(where $T^{i i}=\delta_{i j} T^{i j}$ ). These definitions are such that $\sigma, \sigma_{i}$ and $\sigma_{i j}$ admit a finite non-zero limit when $c \rightarrow+\infty$ (since $T^{\mu \nu}$ has the dimension of an energy density). In the case of our model of point-particles [stress-energy tensor given by (2.14)], we obtain

$$
\begin{aligned}
\sigma(\mathbf{x}, t) & =\operatorname{Pf}\left(\tilde{\mu}_{1} \Delta_{1}\right)+1 \leftrightarrow 2, \\
\sigma_{i}(\mathbf{x}, t) & =\operatorname{Pf}\left(\mu_{1} v_{1}^{i} \Delta_{1}\right)+1 \leftrightarrow 2, \\
\sigma_{i j}(\mathbf{x}, t) & =\operatorname{Pf}\left(\mu_{1} v_{1}^{i} v_{1}^{j} \Delta_{1}\right)+1 \leftrightarrow 2,
\end{aligned}
$$

where $\Delta_{1} \equiv \Delta\left[\mathbf{x}-\mathbf{y}_{1}(t)\right]$, and where $\mu_{1}$ and $\tilde{\mu}_{1}$ represent some effective masses defined by

$$
\begin{aligned}
& \mu_{1}(\mathbf{x}, t)=\frac{m_{1} c}{\sqrt{-\left[g_{\rho \sigma}\right]_{1} v_{1}^{\rho} v_{1}^{\sigma}}} \cdot \frac{1}{\sqrt{-g(\mathbf{x}, t)}}, \\
& \tilde{\mu}_{1}(\mathbf{x}, t)=\mu_{1}(\mathbf{x}, t)\left[1+\frac{\mathbf{v}_{1}^{2}}{c^{2}}\right] .
\end{aligned}
$$

Note that $\mu_{1}$ and $\tilde{\mu}_{1}$ depend on time and space. Indeed, while the first factor in (3.11a) is clearly a mere function of time through the values of the positions and velocities of the particles at the instant $t$, the second factor $(-g)^{-1 / 2}$ is evaluated at the field point $t, \mathbf{x}$ instead of the source point $t, \mathbf{y}_{1}$. From the non-distributivity of the Hadamard regularization, one is not allowed to replace $(-g)^{-1 / 2}$ by its (regularized) value at the point 1 , even though it is multiplied by a delta-pseudo-function at 1 .

\section{B. The $3 P N$ iteration of the metric}

In what follows we sketch the main steps of our iteration of the Einstein field equations (3.3)-(3.8) generated by two particles at the 3PN order. For more clarity in the presentation, we reason by induction over the post-Newtonian order $n$. However, we do not have proved the validity of this method to any order $n$; simply we applied the method outlined below to construct the metric at the $3 \mathrm{PN}$ order.

(I) Suppose by induction over $n$ that we have succeeded in obtaining some approximate post-Newtonian metric coefficients $h_{[2 n-2]}^{\mu \nu}$, as well as the previous coefficients $h_{[m]}^{\mu \nu}$ for any $m$ such that $2 \leq m \leq 2 n-2$, which approach the true metric modulo a small post-Newtonian remainder,

$$
h^{\mu \nu}=h_{[2 n-2]}^{\mu \nu}+\mathcal{O}(2 n-1),
$$

with the notation $\mathcal{O}(2 n-1)=\mathcal{O}\left(1 / c^{2 n-1}\right)$, here and elsewhere, for the post-Newtonian error terms. We assume that the $h_{[m]}^{\mu \nu}$ 's are at once some explicit functions of the field point 
$\mathbf{x}$ and functionals of the two trajectories $\mathbf{y}_{1}(t), \mathbf{y}_{2}(t)$ and velocities $\mathbf{v}_{1}(t), \mathbf{v}_{2}(t)$. Since the matter source of the field equations is made of delta-pseudo-functions, the metric coefficients become singular at the location of the particles (indeed, this is already true at the Newtonian order). As a matter of fact, we assume for the present iteration that,

$$
\forall m \leq 2 n-2, \quad h_{[m]}^{\mu \nu} \in \mathcal{F},
$$

where $\mathcal{F}$ is the class of functions considered in 47,48 and Section [1]. This is not a completely rigorous assumption because of the presence of logarithms in the expansions around the singularities; but we shall see that this assumption is justified at the 3PN order where one can consider these logarithms as mere constants.

(II) Consider for simplicity the combination $\frac{1}{2}\left(h^{00}+h^{i i}\right)$ only, for which we need the maximal post-Newtonian precision since it is directly connected to $g_{00}$. The structure of the Einstein field equations (3.3), containing notably the gravitational source term (3.7)-(3.8), reads as

$$
\square\left(\frac{h^{00}+h^{i i}}{2}\right)=\frac{8 \pi G}{c^{2}}|g| \sigma+\sum h \ldots h \partial h \partial h,
$$

where $\sigma$ is given by (3.9a), where the sum runs over non-linearities and the two partial derivatives $\partial \partial$ have to be distributed among the $h$ 's (with double derivatives allowed in the quadratic term). In order to obtain an equation valid at the next post-Newtonian order $n$, we replace the approximate metric (3.12) into the right-hand side of (3.14). Furthermore, we replace the partial derivatives $\partial \partial$ in (3.14) by the distributional derivatives (2.8) [we shall discuss the effect of using either the particular derivative (2.9) of the more correct one (2.10)]. Using also the density of particles in the form (3.10a), we get

$$
\begin{aligned}
\square\left(\frac{h^{00}+h^{i i}}{2}\right) & =\left\{\frac{8 \pi G}{c^{2}}\left(\operatorname{Pf}|g| \tilde{\mu}_{1} \Delta_{1}+\operatorname{Pf}|g| \tilde{\mu}_{2} \Delta_{2}\right)\right. \\
& \left.+\sum_{m_{1}, \ldots, m_{p} \leq 2 n-2} h_{\left[m_{1}\right]} \ldots h_{\left[m_{p-2}\right]} \partial\left(\operatorname{Pf} h_{\left[m_{p-1}\right]}\right) \partial\left(\operatorname{Pf} h_{\left[m_{p}\right]}\right)\right\}_{[2 n]} \\
& +\mathcal{O}(2 n+1),
\end{aligned}
$$

where the $h_{\left[m_{1}\right]}, \ldots, h_{\left[m_{p}\right]}$ (with $2 \leq p \leq n$ ) denote the metric coefficients known from the previous iterations, and where as indicated by the label $[2 n]$ a truncation up to the postNewtonian order $1 / c^{2 n}$ is understood. At this stage, any subsequent transformation of the right-hand side must be done using the rules for handling the pseudo-functions and their derivatives [47].

(III) We integrate the latter equation by means of the retarded integral given by (3.4):

$$
\frac{h^{00}+h^{i i}}{2}=\square_{\mathcal{R}}^{-1}\left\{\frac{8 \pi G}{c^{2}}\left(\operatorname{Pf}|g| \tilde{\mu}_{1} \Delta_{1}+\operatorname{Pf}|g| \tilde{\mu}_{2} \Delta_{2}\right)\right.
$$




$$
\begin{aligned}
& \left.+\sum_{\substack{m_{1}, \ldots, m_{p} \leq 2 n-2 \\
+\mathcal{O}(2 n+1)}} h_{\left[m_{1}\right]} \ldots h_{\left[m_{p-2}\right]} \partial\left(\operatorname{Pf} h_{\left[m_{p-1}\right]}\right) \partial\left(\operatorname{Pf} h_{\left[m_{p}\right]}\right)\right\}_{[2 n]} \\
& +
\end{aligned}
$$

This defines the solution to the $n$th order, and so, by recursion, to any order (in principle). The partie-finie symbols Pf take care of the divergences of the retarded integral at the locations of the particles; that is, the retarded integral is considered as a partie-finie integral in the sense of [47]. More precisely, the retardations in (3.16) are expanded to the $n \mathrm{PN}$ order and the resulting Poisson-like integrals computed using the duality brackets in the way specified by Section V in 47]. Actually, the Poisson-like integrals, which have a noncompact support, become rapidly divergent at infinity when $n$ increases, and the correct solution we use is not the Poisson-like integral but is obtained by a matching of the inner metric to the multipole expansion of the exterior field. So, in fact,

$$
\begin{aligned}
\left(\square_{\mathcal{R}}^{-1} \operatorname{Pf} F\right)\left(\mathbf{x}^{\prime}, t\right) & =-\frac{1}{4 \pi} \sum_{k=0}^{2 n} \frac{(-)^{k}}{k ! c^{k}}<\left(\frac{\partial}{\partial t}\right)^{k}[\operatorname{Pf} F(\mathbf{x}, t)],\left|\mathbf{x}-\mathbf{x}^{\prime}\right|^{k-1}>_{\text {match }} \\
& +\mathcal{O}(2 n+1)
\end{aligned}
$$

where the subscript "match" refers to the matching process that is described in Section IV in the case of the $3 \mathrm{PN}$ order. Notice that the time-derivatives $(\partial / \partial t)^{k}$ resulting from the Taylor expansion of the retardations are distributional derivatives and therefore can be put outside the duality bracket (see Section IX in [47]). Thus, equivalently,

$$
\begin{aligned}
\left(\square_{\mathcal{R}}^{-1} \operatorname{Pf} F\right)\left(\mathbf{x}^{\prime}, t\right) & =-\frac{1}{4 \pi} \sum_{k=0}^{2 n} \frac{(-)^{k}}{k ! c^{k}}\left(\frac{\partial}{\partial t}\right)^{k}\left[<\operatorname{Pf} F(\mathbf{x}, t),\left|\mathbf{x}-\mathbf{x}^{\prime}\right|^{k-1}>_{\text {match }}\right] \\
& +\mathcal{O}(2 n+1) .
\end{aligned}
$$

(IV) Once the solution (3.16) to the $n$th post-Newtonian order is in hands we perform many simplifications of the expression, following the rules of application of the distributional derivative. In particular, we find very useful to use the fact that a double gradient can be re-expressed in terms of d'Alembertians as

$$
\partial_{\mu} F \partial^{\mu} G=\frac{1}{2}[\square(F G)-F \square G-G \square F],
$$

which implies that the retarded integral reads as

$$
\square_{\mathcal{R}}^{-1}\left[\partial_{\mu} F \partial^{\mu} G\right]=\frac{1}{2} F G-\frac{1}{2} \square_{\mathcal{R}}^{-1}[F \square G+G \square F] .
$$

The first term is "all-integrated", while the second term, in which one can replace the d'Alembertians by their corresponding sources, brings in general many interesting cancellations with other terms. Unfortunately, the formula (3.19) is valid only in an ordinary sense 
but not in the distributional sense, because the distributional derivative does not satisfy, in general, the Leibniz rule. Thus, in general,

$$
\partial_{\mu}(\operatorname{Pf} F) \partial^{\mu}(\operatorname{Pf} G) \neq \frac{1}{2}[\square(\operatorname{Pf} F G)-F \square(\operatorname{Pf} G)-G \square(\operatorname{Pf} F)] .
$$

Nevertheless, the strategy we have chosen to follow in this paper is to take advantage of the many simplifications brought about by the latter process, at the price of introducing some extra terms (named "Leibniz") accounting for the violation of the Leibniz rule. This means that we shall write, similarly to (3.20),

$$
\square_{\mathcal{R}}^{-1}\left[\partial_{\mu}(\operatorname{Pf} F) \partial^{\mu}(\operatorname{Pf} G)\right]=\frac{1}{2} \operatorname{Pf} F G-\frac{1}{2} \square_{\mathcal{R}}^{-1}[F \square(\operatorname{Pf} G)+G \square(\operatorname{Pf} F)]+\delta_{\text {Leibniz }} T,
$$

where the Leibniz term is given by

$$
\delta_{\text {Leibniz }} T=\square_{\mathcal{R}}^{-1}\left[\partial_{\mu}(\operatorname{Pf} F) \partial^{\mu}(\operatorname{Pf} G)-\frac{1}{2} \square(\operatorname{Pf} F G)+\frac{1}{2} F \square(\operatorname{Pf} G)+\frac{1}{2} G \square(\operatorname{Pf} F)\right] .
$$

Obviously the Leibniz term depends only on the purely distributional part of the derivative. See the Appendix A for the complete list of the Leibniz terms. As it will turn out these terms are not too difficult to compute, and, of course, arise precisely at the 3PN order. They give a contribution to the metric and the equations of motion that we shall be able to check from the requirement of Lorentz invariance (see Section VI).

\section{The 3PN non-linear potentials}

The post-Newtonian iteration sketched in the previous subsection is implemented to the $3 \mathrm{PN}$ order. The computation is long but straightforward. After the simplification process described above we find that the metric is parametrized by certain non-linear potentials, which do not carry a physical signification by themselves, but turn out to be useful in the present computation. The $3 \mathrm{PN}$ metric reads as

$$
\begin{aligned}
g_{00}= & -1+\frac{2}{c^{2}} V-\frac{2}{c^{4}} V^{2}+\frac{8}{c^{6}}\left(\hat{X}+V_{i} V_{i}+\frac{V^{3}}{6}\right) \\
& +\frac{32}{c^{8}}\left(\hat{T}-\frac{1}{2} V \hat{X}+\hat{R}_{i} V_{i}-\frac{1}{2} V V_{i} V_{i}-\frac{1}{48} V^{4}\right)+\mathcal{O}(10), \\
g_{0 i}= & -\frac{4}{c^{3}} V_{i}-\frac{8}{c^{5}} \hat{R}_{i}-\frac{16}{c^{7}}\left(\hat{Y}_{i}+\frac{1}{2} \hat{W}_{i j} V_{j}+\frac{1}{2} V^{2} V_{i}\right)+\mathcal{O}(9), \\
g_{i j}= & \delta_{i j}\left[1+\frac{2}{c^{2}} V+\frac{2}{c^{4}} V^{2}+\frac{8}{c^{6}}\left(\hat{X}+V_{k} V_{k}+\frac{V^{3}}{6}\right)\right]+\frac{4}{c^{4}} \hat{W}_{i j} \\
& +\frac{16}{c^{6}}\left(\hat{Z}_{i j}+\frac{1}{2} V \hat{W}_{i j}-V_{i} V_{j}\right)+\mathcal{O}(8) .
\end{aligned}
$$


We recall our notation for the small post-Newtonian remainders: $\mathcal{O}(n)=\mathcal{O}\left(1 / c^{n}\right)$. The various post-Newtonian orders are parametrized by some potentials which are defined by means of the retarded integral (3.4). At the "Newtonian" and 1PN orders we pose

$$
\begin{aligned}
V & =\square_{\mathcal{R}}^{-1}[-4 \pi G \sigma], \\
V_{i} & =\square_{\mathcal{R}}^{-1}\left[-4 \pi G \sigma_{i}\right],
\end{aligned}
$$

in which the source densities were defined by (3.9). Next, at the $2 \mathrm{PN}$ order, we define

$$
\begin{aligned}
\hat{X} & =\square_{\mathcal{R}}^{-1}\left[-4 \pi G V \sigma_{i i}+\hat{W}_{i j} \partial_{i j} V+2 V_{i} \partial_{t} \partial_{i} V+V \partial_{t}^{2} V+\frac{3}{2}\left(\partial_{t} V\right)^{2}-2 \partial_{i} V_{j} \partial_{j} V_{i}\right], \\
\hat{R}_{i} & =\square_{\mathcal{R}}^{-1}\left[-4 \pi G\left(V \sigma_{i}-V_{i} \sigma\right)-2 \partial_{k} V \partial_{i} V_{k}-\frac{3}{2} \partial_{t} V \partial_{i} V\right], \\
\hat{W}_{i j} & =\square_{\mathcal{R}}^{-1}\left[-4 \pi G\left(\sigma_{i j}-\delta_{i j} \sigma_{k k}\right)-\partial_{i} V \partial_{j} V\right] .
\end{aligned}
$$

Finally, at the $3 \mathrm{PN}$ order, we have

$$
\begin{aligned}
\hat{T}= & \square_{\mathcal{R}}^{-1}\left[-4 \pi G\left(\frac{1}{4} \sigma_{i j} \hat{W}_{i j}+\frac{1}{2} V^{2} \sigma_{i i}+\sigma V_{i} V_{i}\right)+\hat{Z}_{i j} \partial_{i j} V+\hat{R}_{i} \partial_{t} \partial_{i} V-2 \partial_{i} V_{j} \partial_{j} \hat{R}_{i}\right. \\
& -\partial_{i} V_{j} \partial_{t} \hat{W}_{i j}+V V_{i} \partial_{t} \partial_{i} V+2 V_{i} \partial_{j} V_{i} \partial_{j} V+\frac{3}{2} V_{i} \partial_{t} V \partial_{i} V+\frac{1}{2} V^{2} \partial_{t}^{2} V \\
& \left.+\frac{3}{2} V\left(\partial_{t} V\right)^{2}-\frac{1}{2}\left(\partial_{t} V_{i}\right)^{2}\right]+\delta_{\text {Leibniz }} \hat{T} \\
\hat{Y}_{i}= & \square_{\mathcal{R}}^{-1}\left[-4 \pi G\left(-\sigma \hat{R}_{i}-\sigma V V_{i}+\frac{1}{2} \sigma_{k} \hat{W}_{i k}+\frac{1}{2} \sigma_{i k} V_{k}+\frac{1}{2} \sigma_{k k} V_{i}\right)+\hat{W}_{k l} \partial_{k l} V_{i}-\partial_{t} \hat{W}_{i k} \partial_{k} V\right. \\
& +\partial_{i} \hat{W}_{k l} \partial_{k} V_{l}-\partial_{k} \hat{W}_{i l} \partial_{l} V_{k}-2 \partial_{k} V \partial_{i} \hat{R}_{k}-\frac{3}{2} V_{k} \partial_{i} V \partial_{k} V-\frac{3}{2} V \partial_{t} V \partial_{i} V \\
& \left.-2 V \partial_{k} V \partial_{k} V_{i}+V \partial_{t}^{2} V_{i}+2 V_{k} \partial_{k} \partial_{t} V_{i}\right]+\delta_{\text {Leibniz }} \hat{Y}_{i} \\
\hat{Z}_{i j}= & \square_{\mathcal{R}}^{-1}\left[-4 \pi G V\left(\sigma_{i j}-\delta_{i j} \sigma_{k k}\right)-2 \partial_{(i} V \partial_{t} V_{j}+\partial_{i} V_{k} \partial_{j} V_{k}+\partial_{k} V_{i} \partial_{k} V_{j}-2 \partial_{(i} V_{k} \partial_{k} V_{j}\right) \\
& \left.-\delta_{i j} \partial_{k} V_{m}\left(\partial_{k} V_{m}-\partial_{m} V_{k}\right)-\frac{3}{4} \delta_{i j}\left(\partial_{t} V\right)^{2}\right]+\delta_{\text {Leibniz }} \hat{Z}_{i j}
\end{aligned}
$$

Note the presence in the $3 \mathrm{PN}$ potentials of the Leibniz contributions described in the previous subsection, which are due to the simplifications we did to arrive at these relatively simple expressions (with respect to what could be expected at the high 3PN order). The Leibniz contributions will be computed in Section $\nabla 1$. Of course, in the case where the matter source is continuous - an hydrodynamical fluid for instance — , the 3PN metric (3.24) and all the expressions of non-linear potentials are valid with simply the Leibniz contributions set to zero.

The potentials (3.25)-(3.27) are connected by the following approximate post-Newtonian differential identities (equivalent to the condition of harmonic coordinates at the 3PN order):

$\partial_{t}\left\{V+\frac{1}{c^{2}}\left[\frac{1}{2} \hat{W}_{k k}+2 V^{2}\right]+\frac{4}{c^{4}}\left[\hat{X}+\frac{1}{2} \hat{Z}_{k k}+\frac{1}{2} V \hat{W}_{k k}+\frac{2}{3} V^{3}\right]\right\}$ 


$$
\begin{gathered}
+\partial_{i}\left\{V_{i}+\frac{2}{c^{2}}\left[\hat{R}_{i}+V V_{i}\right]+\frac{4}{c^{4}}\left[\hat{Y}_{i}-\frac{1}{2} \hat{W}_{i j} V_{j}+\frac{1}{2} \hat{W}_{k k} V_{i}+V \hat{R}_{i}+V^{2} V_{i}\right]\right\}=\mathcal{O}(6), \\
\partial_{t}\left\{V_{i}+\frac{2}{c^{2}}\left[\hat{R}_{i}+V V_{i}\right]\right\}+\partial_{j}\left\{\hat{W}_{i j}-\frac{1}{2} \hat{W}_{k k} \delta_{i j}+\frac{4}{c^{2}}\left[\hat{Z}_{i j}-\frac{1}{2} \hat{Z}_{k k} \delta_{i j}\right]\right\}=\mathcal{O}(4) .
\end{gathered}
$$

We shall check that the (regularized) potentials we compute satisfy these identities. They are in turn respectively equivalent to the equation of continuity at the $2 \mathrm{PN}$ order and the equation of motion at the $1 \mathrm{PN}$ order:

$$
\begin{aligned}
\partial_{t}\left[\sigma\left(1+\frac{2 \hat{W}_{i i}}{c^{4}}\right)\right] & +\partial_{j}\left[\sigma_{j}\left(1+\frac{2 \hat{W}_{i i}}{c^{4}}\right)\right] \\
& =\frac{1}{c^{2}}\left(\partial_{t} \sigma_{j j}-\sigma \partial_{t} V\right)-\frac{4}{c^{4}}\left(\sigma V_{j} \partial_{j} V+\sigma_{j k} \partial_{j} V_{k}\right)+\mathcal{O}(6) \\
\partial_{t}\left[\sigma_{i}\left(1+\frac{4 V}{c^{2}}\right)\right] & +\partial_{j}\left[\sigma_{i j}\left(1+\frac{4 V}{c^{2}}\right)\right] \\
& =\sigma \partial_{i} V+\frac{4}{c^{2}}\left[\sigma \partial_{t} V_{i}+\sigma_{j}\left(\partial_{j} V_{i}-\partial_{i} V_{j}\right)\right]+\mathcal{O}(4)
\end{aligned}
$$

\section{Computing the equations of motion}

The equations of motion of the particle 1 are deduced from the covariant conservation of the stress-energy tensor of the particles,

$$
\nabla_{\nu} T^{\mu \nu}=0
$$

where $T^{\mu \nu}$ is given by the definite expression (2.14) made of the delta-pseudo-functions defined in 47,48]. It is shown in Section $\mathrm{V}$ of [48 that by integrating (3.30) over a volume surrounding the particle 1 (and only 1), i.e. by constructing the duality bracket of (3.30) with the characteristic function of that volume, we obtain the equations of motion of the particle 1 in the form

$$
\frac{d}{d t}\left(\frac{\left[g_{\lambda \mu}\right]_{1} v_{1}^{\mu}}{\sqrt{-\left[g_{\rho \sigma}\right]_{1} \frac{v_{1}^{\rho} v_{1}^{\sigma}}{c^{2}}}}\right)=\frac{1}{2} \frac{\left[\partial_{\lambda} g_{\mu \nu}\right]_{1} v_{1}^{\mu} v_{1}^{\nu}}{\sqrt{-\left[g_{\rho \sigma}\right]_{1} \frac{v_{1}^{\rho} v_{1}^{\sigma}}{c^{2}}}}
$$

These equations of motion take the same form as the geodesic equations for a test particle moving on a smooth background, but with the role of the background metric played by the true metric generated by the two bodies and regularized according to the Lorentzian prescription [48].

In this paper we compute the spatial acceleration of body 1 , which corresponds to the equation with spatial index $\lambda=i$ in (3.31); we do not consider the energy which would 
be given by the equation with time index $\lambda=0$. Indeed, the energy of the binary system will be determined directly from the (fully order-reduced) acceleration. From (3.31) we can write the equations into the form

$$
\frac{d P_{1}^{i}}{d t}=F_{1}^{i},
$$

where the "linear momentum density" $P_{1}^{i}$ and "force density" $F_{1}^{i}$ are given by

$$
\begin{aligned}
P_{1}^{i} & =\frac{\left[g_{i \mu}\right]_{1} v_{1}^{\mu}}{\sqrt{-\left[g_{\rho \sigma}\right]_{1} \frac{v_{1}^{\rho} v_{1}^{\sigma}}{c^{2}}}}, \\
F_{1}^{i} & =\frac{1}{2} \frac{\left[\partial_{i} g_{\mu \nu}\right]_{1} v_{1}^{\mu} v_{1}^{\nu}}{\sqrt{-\left[g_{\rho \sigma}\right]_{1} \frac{v_{1}^{\rho} v_{1}^{\sigma}}{c^{2}}}} .
\end{aligned}
$$

The expressions of both $P_{1}^{i}$ and $F_{1}^{i}$ in terms of the non-linear potentials follow from insertion of the 3PN metric coefficients (3.24). We obtain some complicated sums of products of potentials which are regularized at the point 1 following the prescription $[F]_{1}$. Since the computation will turn out to be quite involved, we decide to adopt the following "step-bystep" strategy:

(A) We compute, in Sections $\square$ and $\mathrm{D}$, all the needed individual potentials and their gradients at the point 1 following the non-Lorentzian regularization $(F)_{1}$; for instance we obtain $\left(\partial_{i} V\right)_{1}$ at the $3 \mathrm{PN}$ order, $(V)_{1}$ at the $2 \mathrm{PN}$ order, $\left(\partial_{i} \hat{X}\right)_{1}$ at the $1 \mathrm{PN}$ order, $\left(\partial_{i} \hat{T}\right)_{1}$ at the Newtonian order, and so on. (Because of the length of the formulas, and since the results for each of these individual regularized potentials are only intermediate, we shall not give them in this paper; see the appendices of [52] for complete expressions.)

(B) We add up the corrections brought about by the Lorentzian regularization $[F]_{1}$ with respect to $(F)_{1}$. We find, at the end of Section $\square$, that the only effect of the new regularization at the 3PN order, when computing the values of potentials at 1 (but the new regularization affects also the corrections due to the non-distributivity), is a crucial 1PN correction arising from the so-called "cubic non-compact" part of $\hat{X}$; that is, we find $\left[\partial_{i} \hat{X}^{(\mathrm{CNC})}\right]_{1}-\left(\partial_{i} \hat{X}^{(\mathrm{CNC})}\right)_{1} \neq 0$.

(C) We replace all the individually regularized potentials $[F]_{1}$ and their gradients into the equations of motion (3.32)-(3.33) which would be obtained while supposing that the Hadamard regularization is "distributive" with respect to the multiplication, i.e. supposing incorrectly that we are allowed to write everywhere $[F G]_{1}=[F]_{1}[G]_{1}$. In doing this we obtain what we call the "distributive" parts of the linear momentum and force densities (3.33), namely $\left(P_{1}^{i}\right)_{\text {distr }}$ and $\left(F_{1}^{i}\right)_{\text {distr }}$. (Other types of non-distributivity arising in the potentials themselves are discussed in Section $\mathbb{\square}$.)

(D) Finally, we compute separately, in Section VI, the corrections due to the nondistributivity, i.e. the differences $P_{1}^{i}-\left(P_{1}^{i}\right)_{\text {distr }}$ and $F_{1}^{i}-\left(F_{1}^{i}\right)_{\text {distr }}$. Note that these corrections 
reflect quantitatively the specific form that we have adopted for the stress-energy tensor of point-particles (2.14). Had we used another stress-energy tensor, for instance by replacing incorrectly $\operatorname{Pf}\left(\frac{1}{\sqrt{-g}} \Delta_{1}\right)$ by $\left[\frac{1}{\sqrt{-g}}\right]_{1} \operatorname{Pf} \Delta_{1}$ inside (2.14), we would have obtained a different nondistributivity, and thereby some different equations of motion. Note also that thanks to the new regularization $[F]_{1}$ the corrections due to the non-distributivity do not alter the Lorentz invariance of the equations of motion. At last, we find the 3PN acceleration of body 1 as

$$
a_{1}^{i}=F_{1}^{i}-\frac{d}{d t}\left(P_{1}^{i}-v_{1}^{i}\right)
$$

We report now the expressions of the distributive parts of the linear momentum and force densities as straightforwardly obtained by substitution of the $3 \mathrm{PN}$ metric (3.24). The expressions of the correcting terms due to the non-distributivity (i.e. $[F G]_{1} \neq[F]_{1}[G]_{1}$ ) are relegated to Section V1, where it is seen that they contribute only at the 3PN order.

$$
\begin{aligned}
& \left(P_{1}^{i}\right)_{\mathrm{distr}}=v_{1}^{i}+\frac{1}{c^{2}}\left(\frac{1}{2} v_{1}^{2} v_{1}^{i}+3[V]_{1} v_{1}^{i}-4\left[V_{i}\right]_{1}\right) \\
& +\frac{1}{c^{4}}\left(\frac{3}{8} v_{1}^{4} v_{1}^{i}+\frac{7}{2}[V]_{1} v_{1}^{2} v_{1}^{i}-4\left[V_{j}\right]_{1} v_{1}^{i} v_{1}^{j}-2\left[V_{i}\right]_{1} v_{1}^{2}\right. \\
& \left.+\frac{9}{2}[V]_{1}^{2} v_{1}^{i}-4[V]_{1}\left[V_{i}\right]_{1}+4\left[\hat{W}_{i j}\right]_{1} v_{1}^{j}-8\left[\hat{R}_{i}\right]_{1}\right) \\
& +\frac{1}{c^{6}}\left(\frac{5}{16} v_{1}^{6} v_{1}^{i}+\frac{33}{8}[V]_{1} v_{1}^{4} v_{1}^{i}-\frac{3}{2}\left[V_{i}\right]_{1} v_{1}^{4}-6\left[V_{j}\right]_{1} v_{1}^{i} v_{1}^{j} v_{1}^{2}+\frac{49}{4}[V]_{1}^{2} v_{1}^{2} v_{1}^{i}\right. \\
& +2\left[\hat{W}_{i j}\right]_{1} v_{1}^{j} v_{1}^{2}+2\left[\hat{W}_{j k}\right]_{1} v_{1}^{i} v_{1}^{j} v_{1}^{k}-10[V]_{1}\left[V_{i}\right]_{1} v_{1}^{2}-20[V]_{1}\left[V_{j}\right]_{1} v_{1}^{i} v_{1}^{j} \\
& -4\left[\hat{R}_{i}\right]_{1} v_{1}^{2}-8\left[\hat{R}_{j}\right]_{1} v_{1}^{i} v_{1}^{j}+\frac{9}{2}[V]_{1}^{3} v_{1}^{i}+12\left[V_{j}\right]_{1}\left[V_{j}\right]_{1} v_{1}^{i} \\
& +12\left[\hat{W}_{i j}\right]_{1}[V]_{1} v_{1}^{j}+12[\hat{X}]_{1} v_{1}^{i}+16\left[\hat{Z}_{i j}\right]_{1} v_{1}^{j}-10[V]_{1}^{2}\left[V_{i}\right]_{1} \\
& \left.-8\left[\hat{W}_{i j}\right]_{1}\left[V_{j}\right]_{1}-8[V]_{1}\left[\hat{R}_{i}\right]_{1}-16\left[\hat{Y}_{i}\right]_{1}\right)+\mathcal{O}(8), \\
& \left(F_{1}^{i}\right)_{\operatorname{distr}}=\left[\partial_{i} V\right]_{1}+\frac{1}{c^{2}}\left(-[V]_{1}\left[\partial_{i} V\right]_{1}+\frac{3}{2}\left[\partial_{i} V\right]_{1} v_{1}^{2}-4\left[\partial_{i} V_{j}\right]_{1} v_{1}^{j}\right) \\
& +\frac{1}{c^{4}}\left(\frac{7}{8}\left[\partial_{i} V\right]_{1} v_{1}^{4}-2\left[\partial_{i} V_{j}\right]_{1} v_{1}^{j} v_{1}^{2}+\frac{9}{2}[V]_{1}\left[\partial_{i} V\right]_{1} v_{1}^{2}\right. \\
& +2\left[\partial_{i} \hat{W}_{j k}\right]_{1} v_{1}^{j} v_{1}^{k}-4\left[V_{j}\right]_{1}\left[\partial_{i} V\right]_{1} v_{1}^{j}-4[V]_{1}\left[\partial V_{j}\right]_{1} v_{1}^{j} \\
& \left.-8\left[\partial_{i} \hat{R}_{j}\right]_{1} v_{1}^{j}+\frac{1}{2}[V]_{1}^{2}\left[\partial_{i} V\right]_{1}+8\left[V_{j}\right]_{1}\left[\partial_{i} V_{j}\right]_{1}+4\left[\partial_{i} \hat{X}\right]_{1}\right) \\
& +\frac{1}{c^{6}}\left(\frac{11}{16} v_{1}^{6}\left[\partial_{i} V\right]_{1}-\frac{3}{2}\left[\partial_{i} V_{j}\right]_{1} v_{1}^{j} v_{1}^{4}+\frac{49}{8}[V]_{1}\left[\partial_{i} V\right]_{1} v_{1}^{4}+\left[\partial_{i} \hat{W}_{j k}\right]_{1} v_{1}^{2} v_{1}^{j} v_{1}^{k}\right. \\
& -10\left[V_{j}\right]_{1}\left[\partial_{i} V\right]_{1} v_{1}^{2} v_{1}^{j}-10[V]_{1}\left[\partial_{i} V_{j}\right]_{1} v_{1}^{2} v_{1}^{j}-4\left[\partial_{i} \hat{R}_{j}\right]_{1} v_{1}^{2} v_{1}^{j} \\
& +\frac{27}{4}[V]_{1}^{2}\left[\partial_{i} V\right]_{1} v_{1}^{2}+12\left[V_{j}\right]_{1}\left[\partial_{i} V_{j}\right]_{1} v_{1}^{2}+6\left[\hat{W}_{j k}\right]_{1}\left[\partial_{i} V\right]_{1} v_{1}^{j} v_{1}^{k} \\
& +6[V]_{1}\left[\partial_{i} \hat{W}_{j k}\right]_{1} v_{1}^{j} v_{1}^{k}+6\left[\partial_{i} \hat{X}\right]_{1} v_{1}^{2}+8\left[\partial_{i} \hat{Z}_{j k}\right]_{1} v_{1}^{j} v_{1}^{k}-20\left[V_{j}\right]_{1}[V]_{1}\left[\partial_{i} V\right]_{1} v_{1}^{j}
\end{aligned}
$$




$$
\begin{aligned}
& -10[V]_{1}^{2}\left[\partial_{i} V_{j}\right]_{1} v_{1}^{j}-8\left[V_{k}\right]_{1}\left[\partial_{i} \hat{W}_{j k}\right]_{1} v_{1}^{j}-8\left[\hat{W}_{j k}\right]_{1}\left[\partial_{i} V_{k}\right]_{1} v_{1}^{j} \\
& -8\left[\hat{R}_{j}\right]_{1}\left[\partial_{i} V\right]_{1} v_{1}^{j}-8[V]_{1}\left[\partial_{i} \hat{R}_{j}\right]_{1} v_{1}^{j}-16\left[\partial_{i} \hat{Y}_{j}\right]_{1} v_{1}^{j}-\frac{1}{6}[V]_{1}^{3}\left[\partial_{i} V\right]_{1} \\
& -4\left[V_{j}\right]_{1}\left[V_{j}\right]_{1}\left[\partial_{i} V\right]_{1}+16\left[\hat{R}_{j}\right]_{1}\left[\partial_{i} V_{j}\right]_{1}+16\left[V_{j}\right]_{1}\left[\partial_{i} \hat{R}_{j}\right]_{1}-8[V]_{1}\left[V_{j}\right]_{1}\left[\partial_{i} V_{j}\right]_{1} \\
& \left.-4[\hat{X}]_{1}\left[\partial_{i} V\right]_{1}-4[V]_{1}\left[\partial_{i} \hat{X}\right]_{1}+16\left[\partial_{i} \hat{T}\right]_{1}\right)+\mathcal{O}(8) .
\end{aligned}
$$

Recall that it is supposed that all the accelerations appearing in the potentials have been order-reduced by means of the equations of motion. Notably, during the reduction of the "Newtonian" term $\left[\partial_{i} V\right]_{1}$ in Section [V], we shall need the equations of motion to the $2 \mathrm{PN}$ order. Furthermore, we see from (3.35a) that when computing the time-derivative of $P_{i}$ we meet an acceleration at $1 \mathrm{PN}$ which is thus also to be replaced by the $2 \mathrm{PN}$ equations of motion. We recall here that the latter $2 \mathrm{PN}$ (or, rather, 2.5PN) equations in harmonic coordinates are 24, 26, 31]

$$
\begin{aligned}
& \frac{d v_{1}^{i}}{d t}=- \frac{G m_{2}}{r_{12}^{2}} n_{12}^{i}+\frac{G m_{2}}{r_{12}^{2} c^{2}}\left\{v_{12}^{i}\left[4\left(n_{12} v_{1}\right)-3\left(n_{12} v_{2}\right)\right]\right. \\
&\left.+n_{12}^{i}\left[-v_{1}^{2}-2 v_{2}^{2}+4\left(v_{1} v_{2}\right)+\frac{3}{2}\left(n_{12} v_{2}\right)^{2}+5 \frac{G m_{1}}{r_{12}}+4 \frac{G m_{2}}{r_{12}}\right]\right\} \\
&+ \frac{G m_{2}}{r_{12}^{2} c^{4}} n_{12}^{i}\left\{\left[-2 v_{2}^{4}+4 v_{2}^{2}\left(v_{1} v_{2}\right)-2\left(v_{1} v_{2}\right)^{2}+\frac{3}{2} v_{1}^{2}\left(n_{12} v_{2}\right)^{2}+\frac{9}{2} v_{2}^{2}\left(n_{12} v_{2}\right)^{2}\right.\right. \\
&\left.-6\left(v_{1} v_{2}\right)\left(n_{12} v_{2}\right)^{2}-\frac{15}{8}\left(n_{12} v_{2}\right)^{4}\right] \\
&+\frac{G m_{1}}{r_{12}}\left[-\frac{15}{4} v_{1}^{2}+\frac{5}{4} v_{2}^{2}-\frac{5}{2}\left(v_{1} v_{2}\right)\right. \\
&\left.+\frac{39}{2}\left(n_{12} v_{1}\right)^{2}-39\left(n_{12} v_{1}\right)\left(n_{12} v_{2}\right)+\frac{17}{2}\left(n_{12} v_{2}\right)^{2}\right] \\
&+\frac{G m_{2}}{r_{12}}\left[4 v_{2}^{2}-8\left(v_{1} v_{2}\right)+2\left(n_{12} v_{1}\right)^{2}-4\left(n_{12} v_{1}\right)\left(n_{12} v_{2}\right)-6\left(n_{12} v_{2}\right)^{2}\right] \\
&+\left.\frac{G G^{2}}{r_{12}^{2}}\left[-\frac{57}{4} m_{1}^{2} m_{2}-9 m_{2}^{2}-\frac{69}{2} m_{12} m_{2}\right]\right\} \\
&+ \frac{G m_{2}}{r_{12}^{2} c^{4}} v_{12}^{i}\left\{n_{12}^{i}\left(n_{12} v_{12}\right)\left[-6 \frac{G m_{1}}{r_{12}}+\frac{52}{3} \frac{G m_{2}}{r_{12}}+3 v_{12}^{2}\right]\right. \\
&+ 4\left(v_{1} v_{2}\right)\left(n_{12} v_{2}\right)-6\left(n_{12} v_{1}\right)\left(n_{12} v_{2}\right)^{2}+\frac{93}{2}\left(n_{12} v_{2}\right)^{3} \\
&\left.\left.+n_{12} v_{1}\right)+\frac{55}{4}\left(n_{12} v_{2}\right)\right]+\frac{G m_{2}}{r_{12}}\left[-2\left(n_{12} v_{1}\right)-2\left(n_{12} v_{2}\right)\right] \\
&\left.+n_{12} v_{2}\right)-4\left(v_{1} v_{2}\right)\left(n_{12} v_{1}\right)
\end{aligned}
$$




$$
\left.+v_{12}^{i}\left[2 \frac{G m_{1}}{r_{12}}-8 \frac{G m_{2}}{r_{12}}-v_{12}^{2}\right]\right\}+\mathcal{O}(6) .
$$

Unavoidably, because of the proliferation of possible terms, the equations of motion at the next 3PN order are even much longer [see (7.16) below].

\section{COMPACT SUPPORT AND QUADRATIC POTENTIALS}

All the potentials that enter the linear momentum $\left(P_{1}^{i}\right)_{\text {distr }}$ and the force density $\left(F_{1}^{i}\right)_{\text {distr }}$ are computed at the point 1 by means of the Lorentzian regularization $[F]_{1}$. However, we shall first determine their Hadamard partie finie in the usual sense $(F)_{1}$, i.e. by approaching the singularity in the spatial slice $t=$ const. The difference between the two regularization processes does not affect any compact or quadratic potentials.

\section{A. Iterative computation of compact support potentials}

In this paragraph, we are interested in the compact terms involved in the equation of motion [see (3.35a) and (3.35b)]. According to our previous remark, it is sufficient to evaluate them with the classical Hadamard prescription. We need $\left(\partial_{i} V\right)_{1}$ up to 3PN order; $(V)_{1}$, $\left(V_{i}\right)_{1}$, and $\left(\partial_{i} V_{j}\right)_{1}$ at $2 \mathrm{PN} ;\left(\hat{W}_{i j}^{(\mathrm{C})}\right)_{1},\left(\partial_{i} \hat{W}_{j k}^{(\mathrm{C})}\right)_{1},\left(\hat{R}_{i}^{(\mathrm{C})}\right)_{1},\left(\partial_{i} \hat{R}_{j}^{(\mathrm{C})}\right)_{1}$ and $\left(\partial_{i} \hat{X}^{(\mathrm{C})}\right)_{1}$ at $1 \mathrm{PN}$. The remaining contributions are Newtonian: $\left(\hat{X}^{(\mathrm{C})}\right)_{1},\left(\partial_{i} \hat{T}^{(\mathrm{C})}\right)_{1},\left(\hat{Y}_{i}^{(\mathrm{C})}\right)_{1}$ and $\left(\partial_{i} \hat{Y}_{j}^{(\mathrm{C})}\right)_{1}$. We follow the same classification and nomenclature concerning the various parts of potentials - compact, non-compact, etc. — as in Section II of [31. The compact (C) potentials are generated by sources with (spatially compact) support limited to the particles; for instance, $V^{(\mathrm{C})}=V$, and, from (3.27a),

$$
\hat{T}^{(\mathrm{C})}=\square_{\mathcal{R}}^{-1}\left[-4 \pi G\left(\frac{1}{4} \sigma_{i j} \hat{W}_{i j}+\frac{1}{2} V^{2} \sigma_{i i}+\sigma V_{i} V_{i}\right)\right]
$$

Thus, by definition, the source $S(\mathbf{x}, t)$ of each compact potential $P^{(\mathrm{C})}$ is made of Dirac pseudo-functions, multiplied by some functions of the class $\mathcal{F}$ :

$$
S(\mathbf{x}, t)=\operatorname{Pf}\left(F \Delta_{1}\right)+\operatorname{Pf}\left(G \Delta_{2}\right)
$$

with $F, G \in \mathcal{F}$. As a result, it is in general possible to find an explicit expression of $P^{(\mathrm{C})}$ over the whole space (for any $\mathbf{x}$ ). Besides, the expansion under the integration symbol of the retardation of $S\left(\mathbf{x}^{\prime}, t-\left|\mathrm{x}-\mathrm{x}^{\prime}\right| / c\right)$ as $c$ goes to infinity is perfectly licit, because the integrand has a compact support:

$$
P^{(\mathrm{C})}=-\frac{1}{4 \pi} \sum_{n=0}^{+\infty} \frac{(-)^{n}}{n ! c^{n}} \partial_{t}^{n} \int d^{3} \mathbf{x}^{\prime}\left|\mathbf{x}-\mathbf{x}^{\prime}\right|^{n-1} S\left(\mathbf{x}^{\prime}, t\right)
$$




$$
=-\frac{1}{4 \pi} \sum_{n=0}^{+\infty} \frac{(-)^{n}}{n ! c^{n}} \partial_{t}^{n}\left(\left[F\left|\mathbf{x}-\mathbf{x}^{\prime}\right|^{n-1}\right]_{1}+\left[G\left|\mathbf{x}-\mathbf{x}^{\prime}\right|^{n-1}\right]_{2}\right) .
$$

The sources $S(\mathbf{x}, t)$ are supposed to be known at the current order. This implies to proceed iteratively as explained in [31]. The reader is referred to this paper for more details. In short, we start from the $V$ and $V_{i}$ potentials, whose sources do not depend on any other ones at the lowest order. Indeed, we have $\square V=-4 \pi G \operatorname{Pf}\left(\tilde{\mu}_{1} \Delta_{1}\right)+1 \leftrightarrow 2$ (and similarly for $\left.V_{i}\right)$, where $\tilde{\mu}_{1}=m_{1}+\mathcal{O}(2)$, as it follows from insertion of the "Newtonian" metric into the definition (3.11) of the effective mass. Hence,

$$
\begin{aligned}
V & =G \int \frac{d^{3} \mathbf{x}^{\prime}}{\left|\mathbf{x}-\mathbf{x}^{\prime}\right|}\left\{\operatorname{Pf}\left[\tilde{\mu}_{1} \Delta_{1}\right]-\frac{1}{c} \partial_{t} \operatorname{Pf}\left[\left|\mathbf{x}-\mathbf{x}^{\prime}\right| \tilde{\mu}_{1} \Delta_{1}\right]\right\}+1 \leftrightarrow 2+\mathcal{O}(2) \\
& =\frac{G m_{1}}{r_{1}}-\frac{G}{c} \partial_{t} m_{1}+1 \leftrightarrow 2+\mathcal{O}(2)=\frac{G m_{1}}{r_{1}}+\frac{G m_{2}}{r_{2}}+\mathcal{O}(2)
\end{aligned}
$$

To obtain the regularized metric (at the location of the first body, say), we need the partie finie of the potential $V$ at point $1,(V)_{1}$. Since we use Hadamard regularization, it is simply given by the value of its non-singular part when $\mathbf{x}=\mathbf{y}_{1}$. Here, we find $(V)_{1}=$ $G m_{2} / r_{12}+\mathcal{O}(2)$, with the notation $r_{12}=\left|\mathbf{y}_{1}-\mathbf{y}_{2}\right|$.

The computation of more complicated compact terms necessitates the knowledge of the effective masses $\mu_{1}$ and $\tilde{\mu}_{1}$ beyond the Newtonian approximation. By substituting to $g_{\mu \nu}$ the explicit 3PN expression (3.24) for the metric in the equations (3.11), we get the general forms of both effective masses. As an example, $\tilde{\mu}_{1}$ at $2 \mathrm{PN}$ order reads

$$
\begin{aligned}
\frac{\tilde{\mu}_{1}}{m_{1}} & =1+\frac{1}{c^{2}}\left[-2 V+[V]_{1}+\frac{3}{2} v_{1}^{2}\right] \\
& +\frac{1}{c^{4}}\left[-2 \hat{W}_{i i}+2 V^{2}-2 V[V]_{1}+\frac{3}{2}[V]_{1}^{2}-\left[V^{2}\right]_{1}-3 V v_{1}^{2}+\frac{7}{2}[V]_{1} v_{1}^{2}-4\left[V_{i}\right]_{1} v_{1}^{i}+\frac{7}{8} v_{1}^{4}\right] \\
& +\mathcal{O}(6),
\end{aligned}
$$

where we are careful at distinguishing the potentials computed at the field point $\mathbf{x}$ from those computed at the source point $\mathbf{y}_{1}$, and where we take into account the non-distributivity of the regularization ( $\mu_{1}$ differs only by some numerical coefficients). Thus, as emphasized in Section III, $\mu_{1}$ and $\tilde{\mu}_{1}$ are functions of time and space. Replacing them by the regularized quantities $\left(\mu_{1}\right)_{1},\left(\tilde{\mu}_{1}\right)_{1}$ (and $1 \leftrightarrow 2$ ) is definitely forbidden because, on one side, the partie finie is not distributive, and, on the other side, the usual Hadamard regularization does not coincide with the Lorentzian one. However, this replacement does not modify any compact potentials, with the notable exception of the $3 \mathrm{PN}$ contributions in $V$ (see below). It is thus convenient to pose:

$$
V_{\text {distr }}=\square_{\mathcal{R}}^{-1}\left[-4 \pi G\left(\tilde{\mu}_{1}\right)_{1} \operatorname{Pf} \delta_{1}-4 \pi G\left(\tilde{\mu}_{2}\right)_{2} \operatorname{Pf} \delta_{2}\right]
$$

and to calculate $V_{\text {distr }}$ and $V-V_{\text {distr }}$ separately. In the other compact sources, we shall employ $\left(\mu_{1}\right)_{1},\left(\tilde{\mu}_{1}\right)_{1}$, etc. instead of $\mu_{1}$ and $\tilde{\mu}_{1}$ for practical calculations at the 3PN approximation. 
Furthermore, in all the compact terms, the action of the Lorentzian delta-pseudo-functions $\operatorname{Pf} \Delta_{1}$ and $\operatorname{Pf} \Delta_{2}$, remarkably, reduces to the one of $\operatorname{Pf} \delta_{1}$ and $\operatorname{Pf} \delta_{2}$. From what precedes, it becomes obvious that, after the evaluation of $\left(\mu_{1}\right)_{1}$ or $\left(\tilde{\mu}_{1}\right)_{1}$ at a given post-Newtonian order $n$, we can determine all the potentials to the precision $1 / c^{2 n}$. As all the terms involving the retarded potentials in $\tilde{\mu}_{1}$ appear already with a factor $1 / c^{2}$ at least, we are then in a position to compute the right-hand-side of the equation (4.4). The process is initiated by the computation of the Newtonian value of $V$ as presented above. Most of the quantities needed to get $\left(\tilde{\mu}_{1}\right)_{1}$ at the $3 \mathrm{PN}$ order are obtained in 31. Finally, the regularized value of $\tilde{\mu}_{1}$ at point 1 is

$$
\begin{aligned}
\frac{\left(\tilde{\mu}_{1}\right)_{1}}{m_{1}}= & 1+\frac{1}{c^{2}}\left[-\frac{G m_{2}}{r_{12}}+\frac{3}{2} v_{1}^{2}\right]+\frac{1}{c^{4}}\left[\frac { G m _ { 2 } } { r _ { 1 2 } } \left(\frac{1}{2} v_{1}^{2}-4\left(v_{1} v_{2}\right)+2 v_{2}^{2}+\frac{1}{2}\left(n_{12} v_{2}\right)^{2}-\frac{1}{2} \frac{G m_{1}}{r_{12}}\right.\right. \\
& \left.\left.+\frac{3}{2} \frac{G m_{2}}{r_{12}}\right)+\frac{7}{8} v_{1}^{4}\right]+\frac{8 G^{2} m_{1} m_{2}}{3 c^{5} r_{12}^{2}}\left(\left(n_{12} v_{1}\right)-\left(n_{12} v_{2}\right)\right) \\
+ & \frac{1}{c^{6}}\left[\frac { G ^ { 2 } m _ { 1 } m _ { 2 } } { r _ { 1 2 } ^ { 2 } } \left(-\frac{3}{2} \frac{G m_{1}}{r_{12}}-\frac{39}{4} \frac{G m_{2}}{r_{12}}+\frac{35}{8} v_{1}^{2}-\frac{41}{4}\left(v_{1} v_{2}\right)+\frac{41}{8} v_{2}^{2}-\frac{9}{8}\left(n_{12} v_{1}\right)^{2}\right.\right. \\
& \left.+\frac{25}{4}\left(n_{12} v_{1}\right)\left(n_{12} v_{2}\right)-\frac{41}{8}\left(n_{12} v_{2}\right)^{2}\right) \\
+ & \frac{G^{2} m_{2}^{2}}{r_{12}^{2}}\left(\frac{3}{2} v_{2}^{2}-\left(n_{12} v_{2}\right)^{2}-3\left(v_{1} v_{2}\right)-\left(n_{12} v_{1}\right)\left(n_{12} v_{2}\right)+\frac{1}{2}\left(n_{12} v_{1}\right)^{2}-\frac{3}{2} \frac{G m_{2}}{r_{12}}+\frac{15}{4} v_{1}^{2}\right) \\
+ & \frac{G m_{2}}{r_{12}}\left(2\left(v_{1} v_{2}\right)^{2}+5 v_{1}^{2} v_{2}^{2}-10\left(v_{1} v_{2}\right) v_{1}^{2}+\frac{33}{8} v_{1}^{4}+2 v_{2}^{4}-\frac{1}{2}\left(n_{12} v_{2}\right)^{2} v_{2}^{2}-\frac{3}{8}\left(n_{12} v_{2}\right)^{4}\right. \\
& \left.\left.-4 v_{2}^{2}\left(v_{1} v_{2}\right)+2\left(n_{12} v_{2}\right)^{2}\left(v_{1} v_{2}\right)-\frac{1}{4} v_{1}^{2}\left(n_{12} v_{2}\right)^{2}\right)+\frac{11}{16} v_{1}^{6}\right]+\mathcal{O}(7) .
\end{aligned}
$$

In our notation, two vectors $\mathbf{v}_{1}, \mathbf{v}_{2}$ between brackets represent the scalar product: $\left(v_{1} v_{2}\right)=$ $v_{1}^{i} v_{2}^{i} ; v_{1}^{2}=v_{1}^{i} v_{1}^{i}$. We recall that it is important to keep the grouping of factors imposed by the regularization in products of potentials. For instance: $\left(V \hat{W}_{i j}\right)_{1} \neq(V)_{1}\left(\hat{W}_{i j}\right)_{1}$.

Among the compact potentials, the $3 \mathrm{PN}$ value of $V$ is certainly the most difficult one to obtain, since the other quantities require only lower orders in powers of $1 / c$. We shall focus on $V_{\text {distr }}$ to illustrate the method we have followed. The difference $V-V_{\text {dist }}$ will be handled in the next subsection. We begin with specializing the general formula for $V$ to the case of $V_{\text {distr }}$ :

$$
\begin{aligned}
V_{\text {distr }}= & \frac{G\left(\tilde{\mu}_{1}\right)_{1}}{r_{1}}-\frac{G}{c} \partial_{t}\left(\tilde{\mu}_{1}\right)_{1}+\frac{G}{2 c^{2}} \partial_{t}^{2}\left[\left(\tilde{\mu}_{1}\right)_{1} r_{1}\right]-\frac{G}{6 c^{3}} \partial_{t}^{3}\left[\left(\tilde{\mu}_{1}\right)_{1} r_{1}^{2}\right]+\frac{G}{24 c^{4}} \partial_{t}^{4}\left[\left(\tilde{\mu}_{1}\right)_{1} r_{1}^{3}\right] \\
& -\frac{G}{120 c^{5}} \partial_{t}^{5}\left[\left(\tilde{\mu}_{1}\right)_{1} r_{1}^{4}\right]+\frac{G}{720 c^{6}} \partial_{t}^{6}\left[\left(\tilde{\mu}_{1}\right)_{1} r_{1}^{5}\right]+1 \leftrightarrow 2+\mathcal{O}(7) .
\end{aligned}
$$

Since the Schwarzschild mass $m_{1}$ is constant, $\partial_{t} \tilde{\mu}_{1} / c$ is of order $\mathcal{O}(3)$ and does not contribute at the $1 \mathrm{PN}$ level. For convenience, we shall introduce some special notation for the terms that occur at this approximation; we pose:

$$
U=\frac{G\left(\tilde{\mu}_{1}\right)_{1}}{r_{1}}+\frac{G\left(\tilde{\mu}_{2}\right)_{2}}{r_{2}} \quad \text { and } \quad X=G\left(\tilde{\mu}_{1}\right)_{1} r_{1}+G\left(\tilde{\mu}_{2}\right)_{2} r_{2} .
$$


Actually the potentials are to be considered as pseudo-functions and it is understood that there is a symbol Pf in front of them. Notably, the time derivatives appearing in (4.6) are distributional. The regularized effective mass $\left(\tilde{\mu}_{1}\right)_{1}$ as well as the distance to the first body $r_{1}$ depend on time through the trajectories $\mathbf{y}_{1,2}(t)$ and velocities $\mathbf{v}_{1,2}(t)$. We explicit the time-differentiations and obtain, at 1PN,

$$
\begin{aligned}
V_{\mathrm{distr}} & =U+\frac{1}{2 c^{2}} \partial_{t}^{2} X+\mathcal{O}(3) \\
& =\frac{G m_{1}}{r_{1}}\left[1+\frac{1}{c^{2}}\left(-\frac{G m_{2}}{r_{12}}+\frac{3}{2} v_{1}^{2}\right)\right]+\frac{G m_{1}}{2 c^{2}}\left(-a_{1}^{i} \partial_{i} r_{1}+v_{1}^{i} v_{1}^{j} \partial_{i j}^{2} r_{1}\right)+1 \leftrightarrow 2+\mathcal{O}(3) .
\end{aligned}
$$

The accelerations are order-reduced by means of the equations of motion at previous orders. Notably, for computing the $1 \mathrm{PN}$ term $\frac{G}{2 c^{2}} \partial_{t}^{2} \operatorname{Pf}\left[\left(\tilde{\mu}_{1}\right)_{1} r_{1}\right]$ at relative order $3 \mathrm{PN}$, we need the $2 \mathrm{PN}$ acceleration given by (3.36). Once we have got $V_{\text {distr }}$ all over the space, the last stage consists of regularizing it, as well as its gradient, at $\mathbf{x}=\mathbf{y}_{1}$ using the Hadamard partie finie. Now, $V_{\text {distr }}$ can be divided into two parts, $V_{\text {distr }} \mathbf{r}_{1}$ and $V_{\text {distr }} \mathbf{r}_{2}$ corresponding to the sources $-4 \pi G\left(\tilde{\mu}_{1}\right)_{1} \operatorname{Pf} \delta_{1}$ and $-4 \pi G\left(\tilde{\mu}_{2}\right)_{2} \operatorname{Pf} \delta_{2}$ respectively. The first part, $V_{\text {distr }} \mathbf{r}_{1}$, depends on $\mathbf{x}$ through $\mathbf{r}_{1}$ only, and contains many terms that are either singular or vanish when $\mathbf{x} \rightarrow \mathbf{y}_{1}$, giving no contribution to the partie finie; on the opposite, the smooth terms with odd $1 / c$-power factors in (4.6) generally contribute. The part $V_{\text {distr } \mathbf{r}_{2}}$ does not necessitate any regularization since it is already regular in the neighbourhood of $\mathbf{x}=\mathbf{y}_{1}$.

The remaining potentials are determined in the same way. However, we have to apply properly the formalism developed in [47]. In particular:

(1) The regularized value of some potential $P^{(\mathrm{C})}$ is the partie finie of $P^{(\mathrm{C})}$ computed initially outside the singularity. In the case where $P^{(\mathrm{C})}$ is the Poisson integral of a compact source $\operatorname{Pf}\left(F \delta_{1}\right)$, with $F \in \mathcal{F}$, we must take care [47] that

$$
P_{1}^{(\mathrm{C})}=\left(\operatorname{Pf} \int \frac{d^{3} \mathbf{x}^{\prime}}{-4 \pi} \frac{1}{\left|\mathbf{x}-\mathbf{x}^{\prime}\right|} F \delta_{1}\right)_{1} \neq \operatorname{Pf} \int \frac{d^{3} \mathbf{x}}{-4 \pi} \frac{1}{r_{1}} F \delta_{1} .
$$

[We generally do not write the dependence of the integrand on the integration variable, as it is evident from the context; thus, $F \delta_{1}$ is computed at point $\mathbf{x}^{\prime}$ in the intermediate expression of (4.8) and at point $\mathbf{x}$ in the last one.]

(2) If $F$ is not regular at point 1 , we generally have $\operatorname{Pf}\left(F \delta_{1}\right) \neq(F)_{1} \operatorname{Pf} \delta_{1}$, even when both members act, in the sense of pseudo-functions, on smooth test functions. This distinction is crucial, for instance, in the determination of $\hat{T}^{(\mathrm{C})}$ at Newtonian order. Indeed, one of its contributions [first term in (4.1)], denoted by $\hat{T}^{(\mathrm{C} 1)}$, reads as:

$$
\begin{aligned}
\hat{T}^{(\mathrm{C} 1)} & =\frac{1}{4} G \operatorname{Pf} \int \frac{d^{3} \mathbf{x}^{\prime}}{\left|\mathbf{x}-\mathbf{x}^{\prime}\right|} \sigma_{i j} \hat{W}_{i j}+\mathcal{O}(1) \\
& =\frac{1}{4} G m_{1} v_{1}^{i} v_{1}^{j}\left(\frac{\hat{W}_{i j}}{\left|\mathbf{x}-\mathbf{x}^{\prime}\right|}\right)_{1}+1 \leftrightarrow 2+\mathcal{O}(1),
\end{aligned}
$$


which is different from $G m_{1} v_{1}^{i} v_{1}^{j}\left(\hat{W}_{i j}\right)_{1} / 4 r_{1}+1 \leftrightarrow 2+\mathcal{O}(1)$. Had we used the latter expression instead of $\hat{T}^{(\mathrm{C} 1)}$, we would have obtained a different potential $\hat{T}_{\text {distr }}$; this would have been correct if the partie finie operation had been "distributive" (see Section II), but we have actually

$$
\hat{T}-\hat{T}_{\text {distr }}=\frac{G^{3} m_{1}^{3}}{r_{1}^{3}}\left[-\frac{1}{240} v_{1}^{2}+\frac{1}{80}\left(n_{1} v_{1}\right)^{2}\right]+1 \leftrightarrow 2 .
$$

Notice that the latter expression is not Galilean-invariant by itself, and therefore will be checked later when verifying that the final equations of motion stay invariant under Lorentz transformations.

\section{B. Non-distributivity in the potential $V$}

We call non-distributivity in the potential $V$ that contribution which arises because the coefficient of the delta-pseudo-function $\operatorname{Pf} \Delta_{1}$ in the matter stress-energy tensor (2.14) is a function not only on time but also on space through the factor $1 / \sqrt{-g}$. It will turn out that this contribution is purely of order $3 \mathrm{PN}$. A related contribution, due to the non-distributivity in $\hat{T}$, has just been computed in the previous subsection. The potential $V$ is generated by the source density $\sigma(\mathbf{x}, t)=\tilde{\mu}_{1} \Delta\left[\mathbf{x}-\mathbf{y}_{1}(t)\right]+1 \leftrightarrow 2$, where $\tilde{\mu}_{1}$ is a function of space-time given explicitly by

$$
\tilde{\mu}_{1}(\mathbf{x}, t)=\frac{m_{1} c\left(1+\frac{\mathbf{v}_{1}^{2}}{c^{2}}\right)}{\sqrt{-\left[g_{\rho \sigma}\right]_{1} v_{1}^{\rho} v_{1}^{\sigma}}} \cdot \frac{1}{\sqrt{-g(\mathbf{x}, t)}} .
$$

The first factor is a function of time, and the second one depends on both time and space (non-distributivity). The potential $V$ is given by the retarded integral (3.25a), whose retardations we expand up to any post-Newtonian order:

$$
V(\mathbf{x}, t)=G \sum_{n=0}^{+\infty} \frac{(-)^{n}}{n ! c^{n}} \partial_{t}^{n} \int d^{3} \mathbf{x}^{\prime}\left|\mathbf{x}-\mathbf{x}^{\prime}\right|^{n-1} \sigma\left(\mathbf{x}^{\prime}, t\right) .
$$

(Actually we shall see that an expansion to the $1 \mathrm{PN}$ order is sufficient for our purpose.) We insert into that expression the source density $\sigma$, use the definition of the delta-pseudofunction $\operatorname{Pf}\left(F \Delta_{1}\right)$ given by (2.13), and arrive at

$$
V(\mathbf{x}, t)=G \sum_{n=0}^{+\infty} \frac{(-)^{n}}{n ! c^{n}} \partial_{t}^{n}\left[\tilde{\mu}_{1}\left(\mathbf{x}^{\prime}, t\right)\left|\mathbf{x}-\mathbf{x}^{\prime}\right|^{n-1}\right]_{1}+1 \leftrightarrow 2
$$

Here, the square brackets refer to the Lorentzian Hadamard regularization when $\mathbf{x}^{\prime} \rightarrow \mathbf{y}_{1}$. Using a multipolar expansion, we obtain immediately these brackets as 


$$
\left[\tilde{\mu}_{1}\left(\mathbf{x}^{\prime}, t\right)\left|\mathbf{x}-\mathbf{x}^{\prime}\right|^{n-1}\right]_{1}=\sum_{l=0}^{+\infty} \frac{(-)^{l}}{l !} \partial_{L}\left(r_{1}^{n-1}\right)\left[r^{\prime \prime}{ }_{1}^{\prime}{ }_{1}^{\prime L} \tilde{\mu}_{1}^{\prime}\right]_{1}
$$

where $\tilde{\mu}_{1}^{\prime} \equiv \tilde{\mu}_{1}(\mathbf{x}, t)$. If $\tilde{\mu}_{1}$ were a function of time only, then we see that all the multipolar contributions in the right-hand side of (4.12) but the scalar $l=0$ one would be zero, because of the factor ${r^{\prime}}_{1}^{l}$ with $l \geq 1$ (this is clear with the old regularization, and easily checked to be true with the Lorentzian regularization as well). We defined $V_{\text {distr }}$ as being $V$ but computed with the function of time $\left[\tilde{\mu}_{1}\right]_{1}$ instead of the true $\tilde{\mu}_{1}(\mathbf{x}, t)$. This $V_{\text {distr }}$ is exactly the one which has been computed in the subsection IVA. [It can be checked that up to the 3PN order $\left[\tilde{\mu}_{1}\right]_{1}=\left(\tilde{\mu}_{1}\right)_{1}$.] Therefore, by the previous argument, $V_{\text {distr }}$ is produced entirely by the scalar part $l=0$ in the latter multipolar expansion, so that its complementary to the true potential $V$ reads as

$$
V-V_{\text {distr }}=G \sum_{n=0}^{+\infty} \frac{(-)^{n}}{n ! c^{n}} \frac{\partial^{n}}{\partial t^{n}}\left\{\sum_{l=1}^{+\infty} \frac{(-)^{l}}{l !} \partial_{L}\left(r_{1}^{n-1}\right)\left[r^{\prime l}{ }_{1}^{l} n_{1}^{\prime L} \tilde{\mu}_{1}^{\prime}\right]_{1}\right\}+1 \leftrightarrow 2,
$$

where the sum over $l$ starts with $l=1$. Thus, the problem reduces to the computation of each regularization terms $\left[r_{1}^{\prime l} n_{1}^{\prime L} \tilde{\mu}_{1}^{\prime}\right]_{1}$. Obviously, at a given post-Newtonian order, these terms will all become zero for $l$ larger than a certain value. We find that, up to the $3 \mathrm{PN}$ order, all the regularizations are zero starting at $l=3$, namely $\left[r^{\prime}{ }_{l} n^{\prime}{ }_{1}^{L} \tilde{\mu}_{1}^{\prime}\right]_{1}=\mathcal{O}(8)$ for any $l \geq 3$, while the non-zero values for $l=1,2$ are given by

$$
\begin{aligned}
& {\left[r^{\prime}{ }_{1} n_{1}^{\prime i} \tilde{\mu}_{1}^{\prime}\right]_{1}=3 \frac{G^{3} m_{1}^{3} m_{2}}{c^{6} r_{12}^{2}} n_{12}^{i}+\mathcal{O}(8)} \\
& {\left[r^{\prime}{ }_{2} n_{1}^{\prime i j} \tilde{\mu}_{1}^{\prime}\right]_{1}=\frac{G^{2} m_{1}^{3}}{c^{4}} \delta^{i j}-3 \frac{G^{3} m_{1}^{3} m_{2}}{c^{6} r_{12}} \delta^{i j}+\frac{3}{2} \frac{G^{2} m_{1}^{3}}{c^{6}} v_{1}^{2} \delta^{i j}-\frac{G^{2} m_{1}^{3}}{c^{6}} v_{1}^{i} v_{1}^{j}+\mathcal{O}(8) .}
\end{aligned}
$$

Replacing these results back into (4.13), and using the fact that $\delta^{i j} \partial_{i j} \frac{1}{r_{1}}=0$, leads to the intermediate form

$$
\begin{aligned}
V-V_{\text {distr }} & =-3 \frac{G^{4} m_{1}^{3} m_{2}}{c^{6} r_{12}^{2}} n_{12}^{i} \partial_{i}\left(\frac{1}{r_{1}}\right)-\frac{G^{3} m_{1}^{3}}{2 c^{6}} v_{1}^{i} v_{1}^{j} \partial_{i j}\left(\frac{1}{r_{1}}\right)+\frac{G^{3} m_{1}^{3}}{2 c^{6}} \frac{\partial^{2}}{\partial t^{2}}\left(\frac{1}{r_{1}}\right) \\
& +\mathcal{O}(8)+1 \leftrightarrow 2 .
\end{aligned}
$$

As we see, the non-distributivity of the potential $V$ is a $3 \mathrm{PN}$ effect. Expanding the time derivative in the last term we find that the dependence on the velocity $v_{1}^{i}$ cancels out, which is normal because a velocity-dependent term would violate the Lorentz invariance, in contradiction with our use of the Lorentzian regularization $[F]_{1}$. The final expression is simple:

$$
V-V_{\text {distr }}=-\frac{5}{2} \frac{G^{4} m_{1}^{3} m_{2}}{c^{6} r_{12}^{2}} n_{12}^{i} \partial_{i}\left(\frac{1}{r_{1}}\right)+\mathcal{O}(8)+1 \leftrightarrow 2
$$


The contribution of the non-distributivity in the acceleration of 1 is given by the gradient at 1 as

$$
\left[\partial_{i} V\right]_{1}-\left[\partial_{i} V_{\mathrm{distr}}\right]_{1}=5 \frac{G^{4} m_{1} m_{2}^{3}}{c^{6} r_{12}^{5}} n_{12}^{i}+\mathcal{O}(8)
$$

\section{Computation of quadratic potentials $(\partial V \partial V)$}

By definition, the quadratic potentials are those whose sources are made of products of two compact factors, like $V, V_{i}, W_{i j}^{(\mathrm{C})}$, etc. (or their derivatives, in most of the time). A typical source term for them is of the type " $\partial V \partial V$ ", hence their denomination; for instance

$$
\hat{W}_{i j}^{(\partial V \partial V)}=\square_{\mathcal{R}}^{-1}\left[-\partial_{i} V \partial_{j} V\right]
$$

But the quadratic source terms may also involve other quantities of the same structure, as

it is the case for $\partial_{t} \hat{W}_{i k}^{(\mathrm{C})} \partial_{k} V$ appearing in the source of the potential $\hat{Y}_{i}^{(\partial V \partial V)}[c f(3.27 \mathrm{~b})]$.

\section{Matching to the external field}

The retardation of the compact potentials defining the metric of an isolated fluid can be expanded in powers of $1 / c$ only in the "near zone" $D_{\text {near }}$ of the source, at a distance much smaller than the typical wave length of the emitted radiation. The question then is how to incorporate in the post-Newtonian metric the no-incoming radiation conditions at past null infinity. We achieve this by performing a matching between the post-Newtonian expansion of the metric, adequate in the near zone, and its multipole expansion, valid in the region $D_{\text {ext }}$ exterior to the compact support of the source. Recall that for slowly moving sources, one can always choose $D_{\text {near }}$ and $D_{\text {ext }}$ in such a way that their intersection is not empty: $D_{\text {near }} \cap D_{\text {ext }} \neq \emptyset$ (see e.g. [53). The field $h^{\mu \nu}$ admits a multipole-type expansion $\mathcal{M}\left(h^{\mu \nu}\right)$, in the sense of [54], at every spatial point $\mathbf{x} \in D_{\text {ext }}$. As a matter of fact, it is shown in 55] that the multipole expansion of the exterior field (a vacuum solution of the field equations) that matches, according to the theory of matched asymptotic expansions, to the post-Newtonian expansion in the interior of the source, is given by

$$
\mathcal{M}\left(h^{\mu \nu}\right)=\mathrm{FP}_{B \rightarrow 0} \square_{\mathcal{R}}^{-1}\left[\left(\frac{r}{r_{0}}\right)^{B} \mathcal{M}\left(\Lambda^{\mu \nu}\right)\right]-\frac{4 G}{c^{4}} \sum_{l=0}^{+\infty} \frac{(-)^{l}}{l !} \partial_{L}\left\{\frac{1}{r} \mathcal{H}_{L}^{\mu \nu}(t-r / c)\right\}
$$

(with $L$ a multi-index of order $l \in \mathbb{N}$ ). The multipole moments $\mathcal{H}_{L}^{\mu \nu}$ entering the right-handside read as

$$
\mathcal{H}_{L}^{\mu \nu}(u)=\mathrm{FP}_{B \rightarrow 0} \int d^{3} \mathbf{y}\left(\frac{|\mathbf{y}|}{r_{0}}\right)^{B} y_{L} \bar{\tau}^{\mu \nu}(\mathbf{y}, u)
$$


where $\bar{\tau}^{\mu \nu}$ represents the (formal) post-Newtonian expansion of the complete source term $\tau^{\mu \nu}=|g| T^{\mu \nu}+\frac{c^{4}}{16 \pi G} \Lambda^{\mu \nu}$ of the field equations (3.3). These expressions are defined by analytic continuation in $B$, and the symbol $\mathrm{FP}_{B \rightarrow 0}$ denotes the finite part when $B$ goes to zero of the Laurent expansion of the analytic continuation (we refer to [54,55] for more details about this finite part).

Let us show how we find the "matched" solution of the equation $\square P=S$ at the relative $1 \mathrm{PN}$ order (this is all we shall need in this paper). We neglect all higher-order postNewtonian contributions in the source term $S$, and look for the solution of

$$
\square P=S^{1 \mathrm{PN}}+\mathcal{O}(3)
$$

Since the formula (4.19) results from the properties of the d'Alembertian operator (and is not specific to the field variable $h^{\mu \nu}$ ), we can use it with the replacements of $\mathcal{M}(\Lambda)$ by $\mathcal{M}\left(S^{1 \mathrm{PN}}\right)$ and of $\bar{\tau}$ by $\frac{c^{4}}{16 \pi G} S^{1 \mathrm{PN}}$. Thus, the multipole expansion of the solution must satisfy

$$
\begin{aligned}
\mathcal{M}(P) & =\mathrm{FP}_{B \rightarrow 0} \square_{\mathcal{R}}^{-1}\left[\left(\frac{r}{r_{0}}\right)^{B} \mathcal{M}\left(S^{1 \mathrm{PN}}\right)\right]-\frac{1}{4 \pi} \sum_{l=0}^{+\infty} \frac{(-)^{l}}{l !} \partial_{L}\left\{\frac{1}{r} \mathcal{P}_{L}(t-r / c)\right\} \\
& +\mathcal{O}(3)
\end{aligned}
$$

with $\quad \mathcal{P}_{L}\left[S^{1 \mathrm{PN}}\right](u)=\mathrm{FP}_{B \rightarrow 0} \int d^{3} \mathbf{y}\left(\frac{|\mathbf{y}|}{r_{0}}\right)^{B} y_{L} S^{1 \mathrm{PN}}(\mathbf{y}, u)$.

The partie-finie retarded integral of the multipole source $\mathcal{M}\left(S^{1 \mathrm{PN}}\right)$ has to be handled with care. It is not licit to develop when $c \rightarrow+\infty$ the integrand under the integration symbol because the source is not compact supported. The correct formula was shown in [56 to be:

$$
\begin{aligned}
\mathrm{FP}_{B \rightarrow 0} \square_{\mathcal{R}}^{-1} & {\left[\left(\frac{r}{r_{0}}\right)^{B} \mathcal{M}\left(S^{1 \mathrm{PN}}\right)\right] } \\
& =\mathrm{FP}_{B \rightarrow 0} \sum_{k=0}^{+\infty} \frac{1}{(2 k) !}\left(\frac{\partial}{c \partial t}\right)^{2 k} \int \frac{d^{3} \mathbf{x}^{\prime}}{-4 \pi}\left|\mathbf{x}-\mathbf{x}^{\prime}\right|^{2 k-1}\left(\frac{r^{\prime}}{r_{0}}\right)^{B} \mathcal{M}\left(S^{1 \mathrm{PN}}\right) \\
& -\frac{1}{4 \pi} \sum_{l \geq 0} \frac{(-)^{l}}{l !} \hat{\partial}_{L}\left\{\frac{\mathcal{R}_{L}(t-r / c)-\mathcal{R}_{L}(t+r / c)}{2 r}\right\} .
\end{aligned}
$$

The hat on the partial derivatives $\hat{\partial}_{L}$ indicates that the trace has been removed, i.e. $\hat{\partial}_{L}=$ $\operatorname{STF}\left(\partial_{L}\right)$. The $\mathcal{R}_{L}$ functions parametrize the general solution of d'Alembert equations that are smooth near the origin: "antisymmetric" solution as given by the last term in (4.22). We have, more precisely,

$$
\mathcal{R}_{L}(u)=\mathrm{FP}_{B \rightarrow 0} \int d^{3} \mathbf{y} \hat{y}^{L}\left(\frac{|\mathbf{y}|}{r_{0}}\right)^{B} T_{l}(\mathbf{y}, u)
$$

with : $\quad T_{l}(\mathbf{y}, u)=(-)^{l+1} \frac{(2 l+1) ! !}{2^{l} l !} \int_{1}^{+\infty} d z\left(z^{2}-1\right)^{l} \mathcal{M}\left(S^{1 \mathrm{PN}}\right)(\mathbf{y}, u-z|\mathbf{y}| / c)$. 
Here, $\hat{y}^{L}$ denotes the symmetric trace-free tensor associated with $y^{i_{1}} \ldots y^{i_{l}}$, for $l \in \mathbb{N}$. With (4.21) and (4.22) we can write the multipole expansion $\mathcal{M}(P)$ at the $1 \mathrm{PN}$ order as

$$
\begin{aligned}
\mathcal{M}(P) & =\mathrm{FP}_{B \rightarrow 0} \int \frac{d^{3} \mathbf{x}^{\prime}}{-4 \pi} \frac{1}{\left|\mathbf{x}-\mathbf{x}^{\prime}\right|}\left(\frac{r^{\prime}}{r_{0}}\right)^{B} \mathcal{M}\left(S^{1 \mathrm{PN}}\right)-\frac{1}{4 \pi} \sum_{l=0}^{+\infty} \frac{(-)^{l}}{l !} \partial_{L}\left(\frac{1}{r}\right) \mathcal{P}_{L}(t) \\
& +\frac{1}{2 c^{2}}\left(\frac{\partial}{\partial t}\right)^{2}\left\{\mathrm{FP}_{B \rightarrow 0} \int \frac{d^{3} \mathbf{x}^{\prime}}{-4 \pi}\left|\mathbf{x}-\mathbf{x}^{\prime}\right|\left(\frac{r^{\prime}}{r_{0}}\right)^{B} \mathcal{M}\left(S^{1 \mathrm{PN}}\right)-\frac{1}{4 \pi} \sum_{l=0}^{+\infty} \frac{(-)^{l}}{l !} \partial_{L}(r) \mathcal{P}_{L}(t)\right\} \\
& +\frac{1}{4 \pi c}[\dot{\mathcal{R}}(t)+\dot{\mathcal{P}}(t)]+\mathcal{O}(3)
\end{aligned}
$$

where the last term, of order $1 / c$, is a simple function of time made of the functions $\mathcal{R}(t)$ and $\mathcal{P}(t)$ defined as being $\mathcal{R}_{L}(t)$ and $\mathcal{P}_{L}(t)$ with $l=0$ (the dot indicates the time-derivative). Now, it can be shown that the latter multipole expansion can be re-written under the new form

$$
\mathcal{M}(P)=\mathcal{M}\left(P^{(\mathrm{I})}\right)+\frac{1}{4 \pi c}[\dot{\mathcal{R}}(t)+\dot{\mathcal{P}}(t)]+\frac{1}{2 c^{2}}\left(\frac{\partial}{\partial t}\right)^{2}\left[\mathcal{M}\left(P^{(\mathrm{II})}\right)\right]+\mathcal{O}(3),
$$

or, equivalently (indeed the second term is a mere function of time, and the multipole expansion obviously commutes with the time derivative),

$$
\mathcal{M}(P)=\mathcal{M}\left(P^{(\mathrm{I})}+\frac{1}{4 \pi c}[\dot{\mathcal{R}}(t)+\dot{\mathcal{P}}(t)]+\frac{1}{2 c^{2}}\left(\frac{\partial}{\partial t}\right)^{2}\left[P^{(\mathrm{II})}\right]\right)+\mathcal{O}(3)
$$

In these equations, $P^{(\mathrm{I})}$ and $P^{(\mathrm{II})}$ denote the matched solutions of the following Poisson equations

$$
\begin{aligned}
\Delta P^{(\mathrm{I})} & =S^{1 \mathrm{PN}}, \\
\Delta P^{(\mathrm{II})} & =2 P^{(\mathrm{I})} .
\end{aligned}
$$

Therefore, we have reduced the problem of finding the matched solution of the d'Alembertian equation (4.20) to that of solving and matching the two successive Poisson equations (4.26a and (4.26b). Now, from the equation (4.25), it is evident that the correct matched solution of (4.20) reads in terms of the matched solutions of (4.26) as

$$
P=P^{(\mathrm{I})}+\frac{1}{4 \pi c}[\dot{\mathcal{R}}(t)+\dot{\mathcal{P}}(t)]+\frac{1}{2 c^{2}}\left(\frac{\partial}{\partial t}\right)^{2}\left[P^{(\mathrm{II})}\right]+\mathcal{O}(3)
$$

To recall the meaning of this solution we shall often denote it as $P=P_{\text {match }}$ below; similarly for $P=P_{\text {match }}^{(\mathrm{I})}$ (for instance $g_{\text {match }}$ computed below) and $P=P_{\text {match }}^{(\mathrm{II})}$ (e.g. $f_{\text {match }}$ ). Actually, we shall find that the function $\mathcal{R}(t)$ appearing in the $1 / c$ term of our solution (4.27) is in fact always either zero or cancelled out by a spatial gradient in the case of the applications made 
in the present paper. Thus, it will not be considered in this paper, whereas the function $\mathcal{P}(t)$ plays a role and is given by

$$
\mathcal{P}(t)=\operatorname{Pf}\left\{\mathrm{FP}_{B \rightarrow 0} \int d^{3} \mathbf{x}\left(\frac{r}{r_{0}}\right)^{B} S^{1 \mathrm{PN}}(\mathbf{x}, t)\right\}
$$

(of course $S^{1 \mathrm{PN}}$ there could be replaced with this approximation by $S^{0.5 \mathrm{PN}}$ ). See (4.41) below for an example of computation of this function.

In practice, in order to find the matched solution of a Poisson equation, $P^{(\mathrm{I})}$ for instance, we proceed as follows. Suppose that we know a particular solution of the equation, say $P_{\text {part }}^{(\mathrm{I})}$. Then the correct solution is necessarily of the type $P^{(\mathrm{I})}=P_{\text {part }}^{(\mathrm{I})}+h_{\mathrm{hom}}^{(\mathrm{I})}$, where $h_{\text {hom }}^{(\mathrm{I})}$ denotes an homogeneous solution of the Laplace equation (harmonic function): $\Delta h_{\mathrm{hom}}^{(\mathrm{I})}=0$, which is moreover regular at the location of the source points. Note that its multipole expansion coincides with itself, $\mathcal{M}\left(h_{\text {hom }}^{(\mathrm{I})}\right)=h_{\text {hom }}^{(\mathrm{I})}$. Now, the latter homogeneous solution is determined by the matching equation as

$$
\begin{aligned}
h_{\text {hom }}^{(\mathrm{I})} & =\mathrm{FP}_{B \rightarrow 0} \int \frac{d^{3} \mathbf{x}^{\prime}}{-4 \pi} \frac{1}{\left|\mathbf{x}-\mathbf{x}^{\prime}\right|}\left(\frac{r^{\prime}}{r_{0}}\right)^{B} \mathcal{M}\left(S^{1 \mathrm{PN}}\right)-\frac{1}{4 \pi} \sum_{l=0}^{+\infty} \frac{(-)^{l}}{l !} \partial_{L}\left(\frac{1}{r}\right) \mathcal{P}_{L}(t) \\
& -\mathcal{M}\left(P_{\text {part }}^{(\mathrm{I})}\right) .
\end{aligned}
$$

It is not evident on that expression that the right-hand side is an harmonic function; but it really is, as can be verified explicitly in practice. We compute the multipole expansion of the source term $S^{1 \mathrm{PN}}$ as well as of our particular solution $P_{\mathrm{part}}^{(\mathrm{I})}$. In our case this means computing the formal expansions of $S^{1 \mathrm{PN}}$ and $P_{\text {part }}^{(\mathrm{I})}$ when $r$ tends to infinity or equivalently when the two source points $\mathbf{y}_{1,2}$ tend to zero. The computation is greatly simplified if one considers the dimensionality of the source. Suppose for instance that $\left[S^{1 \mathrm{PN}}\right]=(\text { length })^{d}$ which means $\left[h_{\mathrm{hom}}^{(\mathrm{I})}\right]=(\text { length })^{d+2}$. Then, using the fact that this function is harmonic, its structure is necessarily of the type $h_{\mathrm{hom}}^{(\mathrm{I})} \sim \sum \hat{x}_{L} y_{1}^{L_{1}} y_{2}^{L_{2}}$ with $l+l_{1}+l_{2}=d+2$. This shows that in order to obtain $h_{\text {hom }}^{(\mathrm{I})}$ completely it is sufficient to develop the right side of (4.29) when $\mathbf{y}_{1,2} \rightarrow 0$ up to the order $d+2$ included (i.e. to control all the terms $y_{1}^{L_{1}} y_{2}^{L_{2}}$ in the expansions which have $\left.l_{1}+l_{2} \leq d+2\right)$. All the higher-order terms, having $l_{1}+l_{2} \geq d+3$ in the right side of (4.29) must manage to give zero. The same method is used to compute the homogeneous solution $h_{\text {hom }}^{(\mathrm{II})}$ contained in $P^{(\mathrm{II})}$. We shall implement this method in practice below.

\section{Structure of the quadratic sources}

In the context of the present paper, we will not need to compute the quadratic sources beyond the $1 \mathrm{PN}$ order. As a consequence, we will deal with only a few kinds of elementary sources. By equation (4.7), we already know the structure of $V$ at the 1PN approximation. 
The other compact retarded potentials have a very similar form. After expansion of the retardation of any of them, say $P^{(\mathrm{C})}$, we get:

$$
\begin{aligned}
P^{(\mathrm{C})} & =\operatorname{Pf} \int \frac{d^{3} \mathbf{x}^{\prime}}{-4 \pi} \frac{1}{\left|\mathbf{x}-\mathbf{x}^{\prime}\right|} F \delta_{1}-\frac{1}{c} \partial_{t} \operatorname{Pf} \int \frac{d^{3} \mathbf{x}}{-4 \pi} F \delta_{1} \\
& +\frac{1}{2 c^{2}} \partial_{t}^{2} \operatorname{Pf} \int \frac{d^{3} \mathbf{x}^{\prime}}{-4 \pi}\left|\mathbf{x}-\mathbf{x}^{\prime}\right| F \delta_{1}+1 \leftrightarrow 2+\mathcal{O}(3),
\end{aligned}
$$

with $F \in \mathcal{F}$. The first contribution has been calculated in [47] [see equation (6.18) there]. What is interesting for us is that the result writes as a sum of space derivative of $1 / r_{1}$ (or $1 / r_{2}$ ), i.e. $\partial_{L}\left(1 / r_{1}\right)$ (with the convention that $L$ designates a multi-index of length $l$ ). Similarly, it is easy to convince oneself that the third contribution is composed of terms $\partial_{L} r_{1}$ (or $\partial_{L} r_{2}$ ). Moreover, the action of time derivatives in front of the integral leaves the latter structure unchanged, in accordance with formulas such as $\partial_{t} \partial_{L} r_{1}=-v_{1}^{i} \partial_{i} \partial_{L} r_{1}$. The second contribution in (4.30) is a mere constant with respect to $\mathbf{x}$. In fact, as they appear in the quadratic sources, the compact potentials are preceded by some space or time derivatives. Now, these derivations have to be performed in the sense of pseudo-functions [47]. From these considerations, we are now in a position to tell what is the precise structure of the sources of quadratic potentials. They read as a sum of what we shall call elementary terms. As we are interested here in their spatial behaviour only, we shall omit purely time dependent factors, though they are normally included. Newtonian elementary terms are themselves products of two pseudo-function derivatives of contributions coming from the first integral in the generic expression (4.30): $\partial_{J} \operatorname{Pf} \partial_{K}\left(1 / r_{1}\right) \times \partial_{L} \operatorname{Pf} \partial_{M}\left(1 / r_{1}\right)$, or $\partial_{J} \operatorname{Pf} \partial_{K}\left(1 / r_{1}\right) \times \partial_{L} \operatorname{Pf} \partial_{M}\left(1 / r_{2}\right)$ (and similarly with $1 \leftrightarrow 2$ ), where $J, K, L, M$ are multi-indices of respective length $j$, $k, l, m$. In the same manner, the $1 \mathrm{PN}$ terms result from products of pseudo-function derivatives of Newtonian and post-Newtonian integrals as the first and the third ones in (4.30): $\partial_{J} \operatorname{Pf} \partial_{K}\left(1 / r_{1}\right) \times \partial_{L} \operatorname{Pf} \partial_{M} r_{1}$, or $\partial_{J} \operatorname{Pf} \partial_{K}\left(1 / r_{1}\right) \times \partial_{L} \operatorname{Pf} \partial_{M} r_{2}$, and $1 \leftrightarrow 2$. As for the $0.5 \mathrm{PN}$ terms, they are simply the pseudo-function derivatives of $\partial_{L}\left(1 / r_{1}\right)$ or $\partial_{L}\left(1 / r_{2}\right)$ (times a mere function of time). It is also natural to distinguish between the "self" elementary terms on one side, which depend on one body only, e.g. $\partial_{i} \operatorname{Pf}\left(1 / r_{1}\right) \times \partial_{j} \operatorname{Pf}\left(1 / r_{1}\right)$, and always admit pre-factors $\mu_{1}^{2}, \tilde{\mu}_{1}^{2}, \tilde{\mu}_{1} \mu_{1}$, and the "interaction" terms on the other side, involving both objects, e.g. $\partial_{i} \operatorname{Pf}\left(1 / r_{1}\right) \times \partial_{j} \operatorname{Pf}\left(1 / r_{2}\right)$. The $0.5 \mathrm{PN}$ terms $\partial_{L}\left(1 / r_{1,2}\right)$ are considered separately.

To be more explicit, we shall provide as an example the $1 \mathrm{PN}$ source of $\hat{W}_{i j}^{(\partial V \partial V)}$ defined by (4.18):

$$
\begin{aligned}
\square \hat{W}_{i j}^{(\partial V \partial V)}= & -G^{2} \tilde{\mu}_{1}^{2} \partial_{i} \operatorname{Pf} \frac{1}{r_{1}} \partial_{j} \operatorname{Pf} \frac{1}{r_{1}}+\frac{G^{2} m_{1}^{2}}{c^{2}}\left(a_{1}^{k} \partial_{(i} \operatorname{Pf} \frac{1}{r_{1}} \partial_{j) k} \operatorname{Pf} r_{1}-v_{1}^{k} v_{1}^{l} \partial_{(i} \operatorname{Pf} \frac{1}{r_{1}} \partial_{j) k l} \operatorname{Pf} r_{1}\right) \\
& -G^{2} \tilde{\mu}_{1} \tilde{\mu}_{2} \partial_{i} \operatorname{Pf} \frac{1}{r_{1}} \partial_{j} \operatorname{Pf} \frac{1}{r_{2}} \\
& +\frac{G^{2} m_{1} m_{2}}{c^{2}}\left(a_{1}^{k} \partial_{(i} \operatorname{Pf} \frac{1}{r_{2}} \partial_{j) k} \operatorname{Pf} r_{1}-v_{1}^{k} v_{1}^{l} \partial_{(i} \operatorname{Pf} \frac{1}{r_{2}} \partial_{j) k l} \operatorname{Pf} r_{1}\right)+1 \leftrightarrow 2+\mathcal{O}(3) .
\end{aligned}
$$

Here, we have used the fact that $\operatorname{Pf} \partial_{i} r_{1}=\partial_{i} \operatorname{Pf} r_{1}$ and $\operatorname{Pf} \partial_{i j} r_{1}=\partial_{i j} \operatorname{Pf} r_{1}$. 
The sum of the retarded integral of the elementary terms then gives us the complete quadratic potentials after expansion in $1 / c$ and matching. Therefore, these potentials are generated by the sources through some partie-finie integrals, which can be regarded as the result of the action of the elementary terms, considered as pseudo-functions, on smooth quantities in the field point. By inspection, it can be shown that the distributional part of the self terms never contributes to the previous integrals, whereas the partie-finie derivatives applied to the interaction terms coincide with those of the Schwartz distribution theory.

\section{Integration of the elementary sources}

We now come to the solving of $\square P=S$ at the 1PN order for each of the elementary terms composing the quadratic sources. We proceed following the method we exposed at the end of paragraph $\mathbb{\nabla C} 1$. For this purpose, we first need to find a particular solution of the following Poisson equations:

$$
\begin{aligned}
\Delta P_{\text {part }}^{(\mathrm{I})} & =\partial_{L} \operatorname{Pf} \frac{1}{r_{1}} \partial_{K} \operatorname{Pf} \frac{1}{r_{1}}, \quad \Delta P_{\text {part }}^{(\mathrm{I})}=\partial_{L} \operatorname{Pf} r_{1} \partial_{K} \operatorname{Pf} \frac{1}{r_{1}}, \\
\text { and } \Delta P_{\text {part }}^{(\mathrm{I})} & =\partial_{L} \operatorname{Pf} \frac{1}{r_{1}} \partial_{K} \operatorname{Pf} \frac{1}{r_{2}}, \quad \Delta P_{\text {part }}^{(\mathrm{I})}=\partial_{L} \operatorname{Pf} r_{1} \partial_{K} \operatorname{Pf} \frac{1}{r_{2}},
\end{aligned}
$$

with $L=i_{1} \ldots i_{l}$ and $K=j_{1} \ldots j_{k}$. From $P_{\text {part }}^{(\mathrm{I})}$, we deduce the matched value $P^{(\mathrm{I})}$ by computing $h_{\text {hom }}^{(\mathrm{I})}$ according to the relation (4.29) adapted for each elementary terms.

The equation (4.32a) involves only the vector variable $\mathbf{r}_{1}$, so that it is simple enough to be integrable in a systematic way. To put the sources into a more suitable form, we start by applying the derivative operator that enters the self terms in the sense of functions, since the purely distributional part of the derivative does not contribute. The result is an adequate power of $r_{1}$ times a finite sum of partial terms $\delta^{i_{1} i_{2}} \ldots \delta^{i_{2 k-1} i_{2 k}} n_{1}^{i_{2 k+1}} \ldots n_{1}^{i_{l}}$, we shall denote more compactly as $\delta^{2 K} n_{1}^{L-2 K}$. The solving of the Poisson equations rests then on the well-known identities (easily checked by direct calculation):

$$
\begin{aligned}
r_{1}^{a} \hat{n}_{1}^{L} & =\Delta\left\{\frac{r_{1}^{a+2} \hat{n}_{1}^{L}}{(a-l+2)(a+l+3)}\right\} \quad \text { for } a \in \mathbb{C} \backslash\{l-2,-l-3\}, \\
r_{1}^{l-2} \hat{n}_{1}^{L} & =\Delta\left\{\frac{1}{2 l+1}\left[\ln \left(\frac{r_{1}}{r_{10}}\right)-\frac{1}{2 l+1}\right] r_{1}^{l} \hat{n}_{1}^{L}\right\}, \\
r_{1}^{-l-3} \hat{n}_{1}^{L} & =\Delta\left\{-\frac{1}{2 l+1}\left[\ln \left(\frac{r_{1}}{r_{10}}\right)+\frac{1}{2 l+1}\right] \frac{\hat{n}_{1}^{L}}{r_{1}^{l+1}}\right\},
\end{aligned}
$$

where $\hat{n}_{1}^{L}$ is the trace-free part of $n_{1}^{L}$, and $r_{10}$ a strictly positive constant. The quantities between braces are particular solutions $P_{\text {part }}^{(\mathrm{I})}$ of $\Delta P^{(\mathrm{I})}=r_{1}^{a} \hat{n}_{1}^{L}$, and we must in general add to them some harmonic functions to be evaluated by matching to the external field. Equations (4.32b) are a priori the most difficult ones, because of the mixing of the sources 1 and 2 . As a matter of fact, determining $P_{\text {part }}^{(\mathrm{I})}$ amounts to solving: 


$$
\Delta g=\frac{1}{r_{1} r_{2}} \quad \Delta f^{12}=\frac{r_{1}}{r_{2}}
$$

in the sense of distributions, on account of the fact that, for instance,

$$
\partial_{L} \operatorname{Pf} \frac{1}{r_{1}} \partial_{K} \operatorname{Pf} \frac{1}{r_{2}}=(-)^{k+l} \partial_{1 L} \partial_{2 K} \operatorname{Pf} \frac{1}{r_{1} r_{2}},
$$

where $\partial_{1 L}$ and $\partial_{2 K}$ denote the partial derivatives with respect to $\mathbf{y}_{1}$ and $\mathbf{y}_{2}$ [the same transformation applies to $\left.\partial_{L} \operatorname{Pf} r_{1} \times \partial_{K} \operatorname{Pf}\left(1 / r_{2}\right)\right]$. As a consequence, ${ }_{L} g_{K} \equiv \partial_{1 L} \partial_{2 K} g$ and ${ }_{L} f_{K}^{12} \equiv \partial_{1 L} \partial_{2 K} f^{12}$ clearly verify:

$$
\Delta\left[(-)^{k+l}{ }_{L} g_{K}\right]=\partial_{L} \operatorname{Pf} \frac{1}{r_{1}} \partial_{K} \operatorname{Pf} \frac{1}{r_{2}} \quad \text { and } \quad \Delta\left[(-)^{k+l}{ }_{L} f_{K}^{12}\right]=\partial_{L} \operatorname{Pf} r_{1} \partial_{K} \operatorname{Pf} \frac{1}{r_{2}}
$$

Note that the derivatives above should be understood as mere (Schwartz) distributional derivatives. Luckily, particular solutions of equations (4.34) in the whole space can be exhibited [57,53,58. We may take:

$$
\begin{aligned}
g & =\ln S, \quad \text { with } S=r_{1}+r_{2}+r_{12}, \\
f^{12} & =-\frac{1}{3} r_{1} r_{12}\left(n_{1} n_{12}\right)\left(g-\frac{1}{3}\right)+\frac{1}{6}\left(r_{2} r_{12}+r_{1} r_{2}-r_{1} r_{12}\right),
\end{aligned}
$$

where $\left(n_{1} n_{12}\right)$ denotes the scalar product of Euclidean vectors. The function $g$ is symmetric in its three variables $\mathbf{x}, \mathbf{y}_{1}$ and $\mathbf{y}_{2}$, so that

$$
\Delta_{1} g=\frac{1}{r_{1} r_{12}} \quad \text { and } \quad \Delta_{2} g=\frac{1}{r_{2} r_{12}}
$$

in the sense of distributions. For a more complete list of useful formulas, see [31]. We have also the two identities:

$$
\Delta_{1} f^{12}=2 g \quad \text { and } \quad \Delta_{2} f^{12}=\frac{r_{12}}{r_{2}} .
$$

Their proof is straightforward and call for some simple relations permitting to express the scalar products $\left(n_{1} n_{2}\right),\left(n_{1} n_{12}\right)$ and $\left(n_{2} n_{12}\right)$ by means of some fractions involving $r_{1}, r_{2}$ and $r_{12}$. These relations, given by (5.14) in [31], are very convenient in most of our computations. The function $f^{12}$ is obtained by exchanging $\mathbf{x}$ and $\mathbf{y}_{1}$ in the function $f$ which was introduced in the appendix $\mathrm{B}$ of [57,

$$
f=\frac{1}{3} r_{1} r_{2}\left(n_{1} n_{2}\right)\left(g-\frac{1}{3}\right)+\frac{1}{6}\left(r_{1} r_{12}+r_{2} r_{12}-r_{1} r_{2}\right),
$$

and which satisfies, in the sense of distributions, the equation

$$
\Delta f=2 g
$$


Once Poisson equations with $1 \mathrm{PN}$ source are integrated, it remains to find the homogeneous solutions to be added to get the full matched solution. Most of the self terms are already correct, namely those that go to zero when $r \rightarrow+\infty$. The other ones are determined from the interaction terms by taking the limit $\mathbf{y}_{2} \rightarrow \mathbf{y}_{1}$, which happens to be always possible. The matching formula (4.29) provides the function $h_{\mathrm{hom}}^{(\mathrm{I})}$ associated to $P_{\mathrm{part}}^{(\mathrm{I})}=g$. The computation is very easy because the dimension of the source is $\left[1 /\left(r_{1} r_{2}\right)\right]=(\text { length })^{-2}$ (i.e. $d=-2$ in the notation of the end of paragraph IV C 1), therefore one needs to control only the constant term in the right side of (4.29) when $\mathbf{y}_{1,2} \rightarrow 0$. We arrive at $h_{\text {hom }}^{(\mathrm{I})}=-\ln \left(2 r_{0}\right)-1$ hence the correct $g_{\text {match }}$ solution of $\Delta g_{\text {match }}=1 / r_{1} r_{2}$ :

$$
g_{\text {match }}=\ln \left(\frac{S}{2 r_{0}}\right)-1
$$

where $r_{0}$ is the positive constant occurring in (4.19). Similarly, but with a little more work because the dimensionality of the source is now $d=0$ so we must expand to second order in $\mathbf{y}_{1,2}$, we obtain the matched value corresponding to $f^{12}$ as

$f_{\text {match }}^{12}=-\frac{1}{3} r_{1} r_{12}\left(n_{1} n_{12}\right)\left(g_{\text {match }}-\frac{1}{3}\right)+\frac{1}{6}\left(r_{2} r_{12}+r_{1} r_{2}-r_{1} r_{12}\right)-\frac{1}{6} r\left(n y_{1}\right)-\frac{1}{6}\left(y_{1} y_{2}\right)+\frac{1}{2} r\left(n y_{2}\right)$

(where $\left(n y_{1}\right)$ for instance denotes the scalar product of $\mathbf{n}=\mathbf{x} / r$ with $\mathbf{y}_{1}$ ). As a consequence, the potentials $P^{(\mathrm{I})}$ satisfying (4.32b) are respectively $(-)^{l+k}{ }_{L} g_{\text {match } K}$ and $(-)^{l+k}{ }_{L} f_{\operatorname{match} K}^{12}$. With this result in hands, we are able to deduce very simply all the self terms that do not match properly yet. We shall content ourselves with examining how this works on an example. Let us suppose we want to solve:

$$
\Delta P^{(\mathrm{I})}=r_{1} \partial_{i j} \operatorname{Pf} \frac{1}{r_{1}}
$$

We make the correspondence with the equation $\Delta f_{\text {match } i j}^{12}=r_{1} \partial_{i j} \operatorname{Pf}\left(1 / r_{2}\right)$, whose source coincides with $r_{1} \partial_{i j} \operatorname{Pf}\left(1 / r_{1}\right)$ for $\mathbf{y}_{1}=\mathbf{y}_{2}$ (we recall our notation $f_{\text {match } i j}^{12}=\partial_{2 i j} f_{\text {match }}^{12}$ ). The distributional part of the derivative yields a compact supported contribution to $f_{\text {match } i j}^{12}$ given by

$$
<\operatorname{Pf} r_{1}\left(\frac{-4 \pi}{3}\right) \delta^{i j} \delta_{2}, \frac{1}{-4 \pi} \frac{1}{\left|\mathbf{x}-\mathbf{x}^{\prime}\right|}>=\frac{r_{12}}{3 r_{2}} \delta^{i j},
$$

which is zero in the limit $\mathbf{y}_{2} \rightarrow \mathbf{y}_{1}$, while the ordinary part yields a Poisson integral which is well-defined and easily evaluated for $\mathbf{y}_{1}=\mathbf{y}_{2}$. We conclude that the value of $f_{\text {match } i j}^{12}$ when $\mathbf{y}_{2} \rightarrow \mathbf{y}_{1}$,

$$
P^{(\mathrm{I})}=\left(f_{\text {match } i j}^{12}\right)_{\mathbf{y}_{2} \rightarrow \mathbf{y}_{1}}=\frac{1}{6} \delta^{i j}-\frac{1}{2} n_{1}^{i} n_{1}^{j},
$$

is precisely the matched solution of the Poisson equation (4.38). 
Let us complete now the program presented at the end of the paragraph IVC 1. Because of the presence of time derivatives at the $1 \mathrm{PN}$ order, we restore in the elementary terms all the coefficients depending only on time, either through the trajectories $\mathbf{y}_{1,2}$ or the velocities $\mathbf{v}_{1,2}$ [we shall generically call $\alpha(t)$ this time-dependent coefficient; for instance $\alpha=\left(v_{1} v_{2}\right)$ ]. It is worth noting that the potentials assimilated to $P^{(\mathrm{II})}$ in (4.26b) are needed only at the Newtonian order, as they come with a $1 / c^{2}$ factor. Consequently, the sources of the $P^{(\mathrm{II})}$ 's are simply (two times) the matched solutions of the Poisson equations $\Delta P^{(\mathrm{I})}=S^{\mathrm{N}}+\mathcal{O}(1)$, where $S^{\mathrm{N}}$ are the Newtonian-type sources $\alpha(t) \partial_{L} \operatorname{Pf}\left(1 / r_{1,2}\right) \times \partial_{K} \operatorname{Pf}\left(1 / r_{1,2}\right)$; so, all we have to solve is:

$$
\begin{aligned}
\Delta P^{(\mathrm{II})} & =2 \alpha(t) r_{1}^{p} \delta^{2 K} n_{1}^{L-2 K}, \\
\text { or } \Delta P^{(\mathrm{II})} & =2 \alpha(t)_{L} g_{\text {match } K},
\end{aligned}
$$

with $p \in \mathbb{Z}$, and $L, K$ some multi-indices. The elementary self potentials obeying (4.39a) are evaluated by application of the identities (4.33), before matching the full term (i.e. included the $\alpha$ coefficients) to the external field. The latter stage will be dropped here, because, on one hand, the general procedure has been explained before, and, on the other hand, powerful methods permitting to deal with the trickiest integrals one could encounter here will be expounded in Section $\nabla$. Let us look next at the second equation (4.39b). To get $P^{(\mathrm{II})}$ from some particular $P_{\text {part }}^{(\mathrm{II})}$ one must perform the complete matching including all the time-dependent factors $\alpha(t)$. Here, for simplicity's sake, we give the result in the case where the (Newtonian) source is $1 /\left(r_{1} r_{2}\right)$ hence $P^{(\mathrm{I})}=g_{\text {match }}$ as we have seen before. Then, we have to find the matched solution of

$$
\Delta P^{(\mathrm{II})}=2 g_{\text {match }} .
$$

A particular solution $P_{\text {part }}^{(\mathrm{II})}$ of this equation is easily obtained with the help of the function $f$ defined by (4.37) [indeed, the Laplacian of $P_{\text {part }}^{(\mathrm{II})}-f$ is a mere constant]. The corresponding homogeneous solution $h_{\text {hom }}^{(\mathrm{II})}$ is computed using the same equation as (4.29) but using the source $2 P^{(\mathrm{I})} \equiv 2 g_{\text {match }}$. The result, that we naturally call $f_{\text {match }} \equiv P^{(\mathrm{II})}$, reads as

$$
\begin{aligned}
f_{\text {match }} & =\frac{1}{3} r_{1} r_{2}\left(n_{1} n_{2}\right)\left(g_{\text {match }}-\frac{1}{3}\right)-\frac{1}{6}\left(r_{1} r_{12}+r_{2} r_{12}-r_{1} r_{2}\right) \\
& -\frac{1}{6} r\left(n y_{1}\right)-\frac{1}{6} r\left(n y_{2}\right)+\frac{1}{2}\left(y_{1} y_{2}\right) .
\end{aligned}
$$

Notice that in the case of the source $1 /\left(r_{1} r_{2}\right)$ the only "odd" contribution $1 / c$ in the formula (4.27) is that given by the function $\mathcal{P}(t)$ defined by (4.28); the contribution due to $\mathcal{R}(t)$ is of higher order in this case. We readily find

$$
\mathcal{P}(t)=\mathrm{FP}_{B \rightarrow 0} \int d^{3} \mathbf{x}\left(\frac{r}{r_{0}}\right)^{B} \frac{1}{r_{1} r_{2}}=-2 \pi r_{12}
$$


(no need of the symbol Pf). This calculation is also done in equation (5.8) of [31]. In Section $\nabla$, we shall see more generally how such integrals can be obtained. Thus, our definitions of $g_{\text {match }}$ and $f_{\text {match }}$ are such that

$$
\left(\square_{\mathcal{R}}^{-1} \frac{1}{r_{1} r_{2}}\right)_{\text {match }}=g_{\text {match }}-\frac{1}{2 c} \dot{r}_{12}+\frac{1}{2 c^{2}} \partial_{t}^{2} f_{\text {match }}+\mathcal{O}(3) .
$$

Finally, we have all the material to integrate the individual post-Newtonian terms in such a way that the inner metric matches to the external field at the $3 \mathrm{PN}$ order. Let us remark however that, in fact, the work we have done on the matching is, as seen a posteriori, unnecessary. Indeed, summing up all the contributions in the potentials, we find that, had we made use of some "un-matched" elementary functions, e.g. $g$ and $f^{12}$ defined by (4.36), to compute the interaction terms instead of the corresponding matched quantities, and had we deduced jointly the corresponding self terms from the limit $\mathbf{y}_{2} \rightarrow \mathbf{y}_{1}$, we would have arrived at the same potentials up to the $3 \mathrm{PN}$ order. This means that the new contributions brought about by the matching to the external field actually cancel out in the final 3PN equations of motion. In particular, the constant $r_{0}$ which enters into the matched quantities $g_{\text {match }}$, $f_{\text {match }}^{12}$ and $f_{\text {match }}$ disappears from the final result. Though we have verified this, we stick to our presentation and use systematically all the matched functions determined previously.

To end this section, we shall achieve the example of the potential $\hat{W}_{i j}^{(\partial V \partial V)}$ defined by (4.18). We indeed already know its source from (4.31). We split the potential itself into:

$$
\hat{W}_{i j}^{(\partial V \partial V)}=-U_{i j}-\frac{1}{c^{2}} K_{i j}+\frac{1}{c} L_{i j}-\frac{1}{2 c^{2}} \partial_{t}^{2} X_{i j}
$$

The first two contributions are respectively the matched solutions of the Poisson equations

$$
\Delta U_{i j}=\partial_{i} U \partial_{j} U \quad \text { and } \quad \Delta K_{i j}=\partial_{(i} U \partial_{j)} \partial_{t}^{2} X
$$

which come from $\partial_{i} U \partial_{j} U+\frac{1}{c^{2}} \partial_{(i} U \partial_{j} \partial_{t}^{2} X+\mathcal{O}(3)=\partial_{i} V \partial_{j} V$. Recall that the potentials $U, X$ and so on have to be viewed as pseudo-functions (for instance $U=\operatorname{Pf} G\left(\tilde{\mu}_{1}\right)_{1} / r_{1}+1 \leftrightarrow 2$ ), so the derivatives entering the source terms are distributional derivatives. The self terms can be determined with the help of the relations (4.33) and matching. To get the interaction part, we change the spatial derivatives to partial derivatives with respect to the source points $\mathbf{y}_{1,2}$, and next, we make the replacement $1 /\left(r_{1} r_{2}\right) \rightarrow g_{\text {match }}, r_{1} / r_{2} \rightarrow f_{\text {match }}^{12}$, and $r_{2} / r_{1} \rightarrow f_{\text {match }}^{21}$. The "odd" term $L_{i j}$ is a pure function of time given by

$$
L_{i j}=\partial_{t} \int \frac{d^{3} \mathbf{x}}{-4 \pi} \partial_{i} U \partial_{j} U
$$

which is already known from equation (5.9) in [31]; it can also be computed with the methods of Section $\nabla$. The contribution $X_{i j}$ is the matched solution of the double-Poisson equation

$$
\Delta^{2} X_{i j}=2 \partial_{i} U \partial_{j} U
$$


whose source is to be considered at the Newtonian order only. The iterative application of (4.33) plus matching yields the self terms; for interaction terms, we replace $\partial_{L}\left(1 / r_{1}\right) \partial_{K}\left(1 / r_{2}\right)$ by $(-)^{l+k} f_{\text {match } K}$. The results are

$$
\begin{aligned}
U_{i j}= & \frac{G^{2} \tilde{\mu}_{1}^{2}}{8}\left(\partial_{i j}^{2} \ln r_{1}+\frac{\delta^{i j}}{r_{1}^{2}}\right)+G^{2} \tilde{\mu}_{1} \tilde{\mu}_{2}{ }_{i} g_{\text {match } j}, \\
K_{i j}= & G^{2} \tilde{\mu}_{1}^{2}\left[-\frac{a_{1}^{(i}}{4} \partial_{j} \ln r_{1}+\frac{a_{1}^{k}}{8} \delta^{i j} \partial_{k} \ln r_{1}-\frac{a_{1}^{k}}{48} \partial_{i j k}\left(r_{1}^{2} \ln r_{1}\right)+\frac{v_{1}^{2} \delta^{i j}}{16 r_{1}^{2}}+\frac{v_{1}^{i} v_{1}^{i}}{8 r_{1}^{2}}-\frac{v_{1}^{k} v_{1}^{l}}{16} \delta^{i j} \partial_{k l}^{2} \ln r_{1}\right. \\
& \left.\quad+\frac{v_{1}^{2}}{16} \partial_{i j} \ln r_{1}+\frac{v_{1}^{k} v_{1}^{l}}{96} \partial_{i j k l}\left(r_{1}^{2} \ln r_{1}\right)\right] \\
+ & G^{2} \tilde{\mu}_{1} \tilde{\mu}_{2}\left[a _ { 1 } ^ { k } k \left(i f_{\text {match } j)}^{12}+v_{1}^{k} v_{1}^{l} k l\left(i f_{\text {match } j)}^{12}\right]+1 \leftrightarrow 2,\right.\right. \\
L_{i j}= & G^{2} \partial_{t}\left[\tilde{\mu}_{1}^{2} \operatorname{Pf} \int \frac{d^{3} \mathbf{x}}{-4 \pi} \partial_{i} \frac{1}{r_{1}} \partial_{j} \frac{1}{r_{1}}\right]+G^{2} \partial_{t}\left[\tilde{\mu}_{1} \tilde{\mu}_{2} \partial_{1 i} \partial_{2 j} \operatorname{Pf} \int \frac{d^{3} \mathbf{x}}{-4 \pi} \frac{1}{r_{1} r_{2}}\right]+1 \leftrightarrow 2+\mathcal{O}(2) \\
= & \left.G^{2} \tilde{\mu}_{1} \tilde{\mu}_{2} \partial_{t} \partial_{1 i} \partial_{2 j} \frac{r_{12}}{2}+1 \leftrightarrow 2+44 \mathrm{~b}\right) \\
X_{i j}= & \frac{G^{2} \tilde{\mu}_{1}^{2}}{4}\left[\frac{1}{6} \partial_{i j}\left(r_{1}^{2} \ln r_{1}\right)+\delta^{i j} \ln r_{1}\right]+G^{2} \tilde{\mu}_{1} \tilde{\mu}_{2} f_{i} f_{\text {match } j}+1 \leftrightarrow 2+\text { const } .
\end{aligned}
$$

In the last equation we do not write for simplicity a constant (associated with a function of type $\mathcal{R}$ ) which is cancelled out by the time derivative $\partial_{t}^{2}$ in front of that term. The self terms have been written in the form of some (ordinary) space derivatives in order to prepare the computation of the cubic sources.

\section{CUBIC POTENTIALS}

\section{A. methodological scheme}

For methodological reasons, it is convenient to express all the cubic sources in a similar way, with the help of the same set of elementary integrals. The so-called "cubic-noncompact" term

$$
\hat{X}^{(\mathrm{CNC})}=\square_{\mathcal{R}}^{-1}\left\{\hat{W}_{i j}^{(\partial V \partial V)} \partial_{i j} V\right\}
$$

which is part of the $\hat{X}$-potential [see (3.26a)], is a good example to understand the successive transformation operations we perform in practice. Furthermore, this cubic-non-compact term is the only one we need to compute at the relative 1PN order; all the other ones, which enter into $\hat{T}$ and $\hat{Y}_{i}$, are merely Newtonian. So the practical computation of (5.1) is the most difficult one we face at the $3 \mathrm{PN}$ approximation. Recall that $\hat{W}_{i j}^{(\partial V \partial V)}$ was defined by (4.18). We start from the expression of the source of $\hat{X}^{(\mathrm{CNC})}$ obtained by insertion of (4.7) and (4.43) into (5.1). We get:

$$
\square \hat{X}^{(\mathrm{CNC})}=-U_{i j} \partial_{i j} U+\frac{1}{c} L_{i j} \partial_{i j} U-\frac{1}{c^{2}} K_{i j} \partial_{i j} U
$$




$$
-\frac{1}{2 c^{2}}\left[\partial_{t}^{2} X_{i j} \partial_{i j} U+U_{i j} \partial_{i j} \partial_{t}^{2} X\right]+\mathcal{O}(3),
$$

using the notation introduced in (4.43). In the right side, the potentials are seen as pseudofunctions (involving a Pf) and the derivatives are distributional. After carrying on the expansion of retardations up to the $1 \mathrm{PN}$ approximation, we find:

$$
\begin{aligned}
\hat{X}^{(\mathrm{CNC})} & =\int \frac{d^{3} \mathbf{x}^{\prime}}{4 \pi} \frac{1}{\left|\mathbf{x}-\mathbf{x}^{\prime}\right|} U_{i j} \partial_{i j} U-\frac{1}{c} \int \frac{d^{3} \mathbf{x}^{\prime}}{4 \pi} \frac{1}{\left|\mathbf{x}-\mathbf{x}^{\prime}\right|} L_{i j} \partial_{i j} U \\
& -\frac{1}{c} \partial_{t} \int \frac{d^{3} \mathbf{x}^{\prime}}{4 \pi} U_{i j} \partial_{i j} U+\frac{1}{c^{2}} \int \frac{d^{3} \mathbf{x}^{\prime}}{4 \pi} \frac{1}{\left|\mathbf{x}-\mathbf{x}^{\prime}\right|} K_{i j} \partial_{i j} U \\
& +\frac{1}{2 c^{2}} \int \frac{d^{3} \mathbf{x}^{\prime}}{4 \pi} \frac{1}{\left|\mathbf{x}-\mathbf{x}^{\prime}\right|} \partial_{t}^{2} X_{i j} \partial_{i j} U+\frac{1}{2 c^{2}} \int \frac{d^{3} \mathbf{x}^{\prime}}{4 \pi} \frac{1}{\left|\mathbf{x}-\mathbf{x}^{\prime}\right|} U_{i j} \partial_{i j} \partial_{t}^{2} X \\
& +\frac{1}{2 c^{2}} \partial_{t}^{2} \int \frac{d^{3} \mathbf{x}^{\prime}}{4 \pi}\left|\mathbf{x}-\mathbf{x}^{\prime}\right| U_{i j} \partial_{i j} U+\frac{1}{c^{2}} \partial_{t} \int \frac{d^{3} \mathbf{x}^{\prime}}{4 \pi} L_{i j} \partial_{i j} U+\mathcal{O}(3) .
\end{aligned}
$$

We have checked explicitly that the sum of the integrals occuring in this formula yields an integral convergent at infinity when considering the regularized value of the gradient $\left(\partial_{i} \hat{X}^{(\mathrm{CNC})}\right)_{1}$ which is the only thing required; thus we do not need to introduce a finite part at infinity (but of course the regularization $\mathrm{Pf}$ is needed to cure the point-particle singularities). The next step consists of replacing the potentials $U, X, L_{i j}, K_{i j}$ and $X_{i j}$ given by (4.44) above by their values at the field point $\mathbf{x} \neq \mathbf{y}_{1}$ and $\mathbf{x} \neq \mathbf{y}_{2}$. The spatial and time derivations appearing in each of the integrals of (5.3) are to be understood in the sense of pseudo-functions (see Section [1]). Consider, as an example, the term

$$
\int \frac{d^{3} \mathbf{x}^{\prime}}{-4 \pi} \frac{1}{\left|\mathbf{x}-\mathbf{x}^{\prime}\right|} K_{i j} \partial_{i j} U
$$

Remind that $K_{i j}$ is given by (4.44b). Let us multiply (4.44b) by $\partial_{i j} U=\partial_{i j} \operatorname{Pf}\left(G \tilde{\mu}_{1} / r_{1}+\right.$ $\left.G \tilde{\mu}_{2} / r_{2}\right)$ and develop the product. The result is made of a sum of terms of the type $\left(1 / r_{1}^{2}\right) \times$ $\partial_{i j} \operatorname{Pf}\left(1 / r_{1}\right), \partial_{k} \ln r_{1} \times \partial_{i j} \operatorname{Pf}\left(1 / r_{1}\right), \partial_{i j k l}\left(r_{2}^{2} \ln r_{2}\right) \times \partial_{i j} \operatorname{Pf}\left(1 / r_{1}\right),{ }_{i k l}\left(f_{\text {match }}^{12}\right)_{j} \times \partial_{i j} \operatorname{Pf}\left(1 / r_{1}\right)$, etc. Some of them are functions of $\mathbf{r}_{1}$ only; we call them "self" terms, whereas those depending on both $\mathbf{r}_{1}$ and $\mathbf{r}_{2}$ are called "interaction" terms.

\section{B. Self terms}

We agree on considering only the self terms (i) that are proportional to $m_{1}^{3}$ rather than $m_{2}^{3}$ (they are the same modulo the replacement $1 \rightarrow 2$ ), (ii) that do contribute to the $1 \mathrm{PN}$

order at most. We leave aside the terms that are generated by $L_{i j}$, since their structure is especially simple and they are evaluated at the end of the section. By explicitly writing down all the sources, as done previously for $K_{i j} \partial_{i j} U$, we can draw the complete list of intervening terms. There are three types of terms: the $V \partial V \partial V$-type concerns one kind of term only, i.e. $1 / r_{1} \times \partial_{i} \operatorname{Pf}\left(1 / r_{1}\right) \times \partial_{j} \operatorname{Pf}\left(1 / r_{1}\right)$; the so-called $\mathcal{Y}$-type refers to $\partial_{L} \operatorname{Pf} r_{1}^{p} \times$ $\partial_{K} \mathrm{Pf} r_{1}^{q}$ terms, where $p$ and $q$ are positive or negative integers; the $\mathcal{N}$-type terms come as 
$\partial_{L} \operatorname{Pf} \partial_{M}\left(r_{1}^{p} \ln r_{1}\right) \times \partial_{J} \operatorname{Pf} \partial_{K} r_{1}^{q}$ (the terms $\mathcal{Y}$ and $\mathcal{N}$ are named after some integrals introduced below). There may exist contracted indices among the set of multi-indices. In particular, some terms involve a factor $\partial \Delta \operatorname{Pf}\left(1 / r_{1}\right)$ and are thus purely compact supported. In fact all the terms can be split into compact and non-compact parts. The latter part is an ordinary function that we are able to calculate explicitly. The former is determined from the results of Sections VI-VIII in [47] and depends on the pseudo-function derivative we use. We shall refer to it as the self partie-finie-derivative contribution to the potentials. If we take the term $\partial_{i j k l}\left(r_{1}^{2} \ln r_{1}\right) \times \partial_{i j} \operatorname{Pf}\left(1 / r_{1}\right)$ for instance, it reads:

$$
\partial_{i j k l}\left(r_{1}^{2} \ln r_{1}\right) \partial_{i j} \mathrm{Pf} \frac{1}{r_{1}}=\partial_{i j k l}\left(r_{1}^{2} \ln r_{1}\right) \partial_{i j} \frac{1}{r_{1}}+\partial_{i j k l}\left(r_{1}^{2} \ln r_{1}\right) \mathrm{D}_{i j}\left[\frac{1}{r_{1}}\right] .
$$

In the case of the "particular" derivative defined by (2.9), we have $\mathrm{D}_{i j}^{\text {part }}\left[1 / r_{1}\right]=2 \pi \operatorname{Pf}\left(\delta^{i j}-\right.$ $\left.5 n_{1}^{i} n_{1}^{j}\right) \delta_{1}$; so that

$$
\partial_{i j k l}\left(r_{1}^{2} \ln r_{1}\right) \partial_{i j} \operatorname{Pf} \frac{1}{r_{1}}=12 \frac{n_{1}^{k} n_{1}^{l}-\delta^{k l}}{r_{1}^{5}}+8 \pi \operatorname{Pf} \frac{-3 n_{1}^{k} n_{1}^{l}+4 \delta^{k l}}{r_{1}^{2}} \delta_{1}
$$

(like in the first term of the right side we sometimes do not write the Pf when there is no possible confusion). In most of this section, we shall use the particular derivative $\mathrm{D}_{i j}^{\text {part }}[F]$ given by (2.9) instead of the more "correct" derivative $\mathrm{D}_{i j}[F]$ defined by (2.10)-(2.11); in Section VI we shall discuss the effect on the final 3PN equations of motion of using the derivative $\mathrm{D}_{i j}[F]$. In order to obtain the self cubic potentials, all we have to do now is to apply the operator $\int \frac{d^{3} \mathbf{x}^{\prime}}{-4 \pi}\left|\mathbf{x}-\mathbf{x}^{\prime}\right|^{-1}$ to the various sources we are focusing on, and $\partial_{t}^{2} \int \frac{d^{3} \mathbf{x}^{\prime}}{-4 \pi}\left|\mathbf{x}-\mathbf{x}^{\prime}\right|$ to the Newtonian source of $\hat{X}^{(\mathrm{CNC})}$. As a matter of fact, the resulting integrals can be viewed as partie finie pseudo-functions like (5.4) acting on $1 /\left(-4 \pi\left|\mathbf{x}-\mathbf{x}^{\prime}\right|\right)$ or $\left|\mathbf{x}-\mathbf{x}^{\prime}\right| /(-4 \pi)$; both quantities are smooth at point 1 , so the pseudo-functions associated with the non-compact part reduce to Schwartz distributions in that case (but, in order to construct the pseudofunctions themselves, we used the generalized distributions of [47]). Each integral is indeed a sum of terms of the form $r_{1}^{p} \delta^{2 K} n_{1}^{L-2 K}$, where $p$ belongs to $\mathbb{Z}$. It is convenient to write them as sums of pseudo-function (or, equivalently here, distributional) derivatives of quantities without indices ("scalars"), times some possible Kronecker symbols. We have for example:

$$
\begin{aligned}
\frac{n_{1}^{i} n_{1}^{j}}{r_{1}^{5}} & =\frac{1}{15} \partial_{i j} \frac{1}{r_{1}^{3}}+\frac{1}{5} \frac{\delta^{i j}}{r_{1}^{5}} \\
& =\frac{1}{15} \partial_{i j} \operatorname{Pf} \frac{1}{r_{1}^{3}}+\frac{1}{5} \frac{\delta^{i j}}{r_{1}^{5}}+\frac{4 \pi}{15}\left(8 n_{1}^{i} n_{1}^{j}-\delta^{i j}\right) \frac{\delta_{1}}{r_{1}^{2}},
\end{aligned}
$$

and other similar formulas for $n_{1}^{i} / r_{1}^{4}, n_{1}^{i} n_{1}^{j} n_{1}^{k} / r_{1}^{6}$, etc.

As an illustration of our handling of the sources, here are the effects of these transformations on $\partial_{i j k l}\left(r_{1}^{2} \ln r_{1}\right) \partial_{i j} \operatorname{Pf}\left(1 / r_{1}\right)$. Starting from equation (5.4) and using (5.5), we find

$$
\int \frac{d^{3} \mathbf{x}^{\prime}}{-4 \pi} \frac{1}{\left|\mathbf{x}-\mathbf{x}^{\prime}\right|} \partial_{i j k l}^{\prime}\left(r_{1}^{\prime 2} \ln r_{1}^{\prime}\right) \partial_{i j}^{\prime} \operatorname{Pf} \frac{1}{r_{1}^{\prime}}
$$




$$
=\int \frac{d^{3} \mathbf{x}^{\prime}}{-4 \pi} \frac{1}{\left|\mathbf{x}-\mathbf{x}^{\prime}\right|}\left[\frac{4}{5} \partial_{k l}^{\prime} \operatorname{Pf} \frac{1}{r_{1}^{\prime 3}}-\frac{48}{5} \operatorname{Pf} \frac{\delta^{k l}}{r_{1}^{\prime 5}}+\frac{8 \pi}{5} \operatorname{Pf} \frac{n_{1}^{\prime k} n_{1}^{\prime l}-18 \delta^{k l}}{r_{1}^{\prime 2}} \delta_{1}^{\prime}\right],
$$

where the first term is generated by the specific derivative (2.9). In this term the derivative can be changed to a partial derivative with respect to the point 1 , and since we employ a pseudo-function derivative, we are allowed to permute integration and derivation symbols. This yields

$$
\begin{aligned}
\operatorname{Pf} \int \frac{d^{3} \mathbf{x}^{\prime}}{-4 \pi} & \frac{1}{\left|\mathbf{x}-\mathbf{x}^{\prime}\right|} \partial_{i j k l}^{\prime}\left(r_{1}^{\prime 2} \ln r_{1}^{\prime}\right) \partial_{i j}^{\prime} \operatorname{Pf} \frac{1}{r_{1}^{\prime}} \\
& =\frac{4}{5} \partial_{1 k l} \operatorname{Pf} \int \frac{d^{3} \mathbf{x}^{\prime}}{-4 \pi} \frac{1}{\left|\mathbf{x}-\mathbf{x}^{\prime}\right|} \frac{1}{r_{1}^{\prime 3}}-\frac{48}{5} \delta^{k l} \operatorname{Pf} \int \frac{d^{3} \mathbf{x}^{\prime}}{-4 \pi} \frac{1}{\left|\mathbf{x}-\mathbf{x}^{\prime}\right|} \frac{1}{r_{1}^{\prime 5}} \\
& +\left(-\frac{2}{5} \frac{n_{1}^{\prime k} n_{1}^{\prime l}}{r_{1}^{\prime 2}\left|\mathbf{x}-\mathbf{x}^{\prime}\right|}+\frac{36}{5} \frac{\delta^{k l}}{r_{1}^{\prime 2}\left|\mathbf{x}-\mathbf{x}^{\prime}\right|}\right)_{1} .
\end{aligned}
$$

The first two terms are left in this form for the time being. On the other hand the last term is computed following the procedure explained by the equations (6.17)-(6.18) in [47]; see also (5.16) below. By implementing the previous procedure for all the self terms entering $K_{i j} \partial_{i j} U$, we finally arrive at:

$$
\begin{gathered}
\left(\int \frac{d^{3} \mathbf{x}^{\prime}}{-4 \pi} \frac{1}{\left|\mathbf{x}-\mathbf{x}^{\prime}\right|} K_{i j} \partial_{i j} U\right)_{\text {self }}=G^{3} m_{1}^{3}\left\{-\frac{a_{1}^{i}}{6} \partial_{1 i} \operatorname{Pf} \int \frac{d^{3} \mathbf{x}^{\prime}}{-4 \pi} \frac{1}{\left|\mathbf{x}-\mathbf{x}^{\prime}\right|} \frac{1}{r_{1}^{\prime 3}}\right. \\
+\frac{v_{1}^{i} v_{1}^{j}}{30} \partial_{1 i j} \operatorname{Pf} \int \frac{d^{3} \mathbf{x}^{\prime}}{-4 \pi} \frac{1}{\left|\mathbf{x}-\mathbf{x}^{\prime}\right|} \frac{1}{r_{1}^{\prime 3}}-\frac{2 v_{1}^{2}}{5} \operatorname{Pf} \int \frac{d^{3} \mathbf{x}^{\prime}}{-4 \pi} \frac{1}{\left|\mathbf{x}-\mathbf{x}^{\prime}\right|} \frac{1}{r_{1}^{\prime 5}} \\
\left.-\frac{\left(n_{1} a_{1}\right)}{12 r_{1}^{2}}+\frac{7\left(n_{1} v_{1}\right)^{2}}{150 r_{1}^{3}}-\frac{7 v_{1}^{2}}{450 r_{1}^{3}}\right\}+1 \leftrightarrow 2+\mathcal{O}(1) .
\end{gathered}
$$

We follow the same way to treat the self parts of the other cubic potentials of interest here: $\hat{T}^{(\mathrm{CNC})}, \hat{Y}_{i}^{(\mathrm{CNC})}$, or the remaining of $\hat{X}^{(\mathrm{CNC})}$.

We find that there are definite contributions, coming at the $3 \mathrm{PN}$ order, due specifically to the pseudo-function derivative introduced in [47]. Indeed, the distributional part of the derivative gives some well-defined non-zero contributions, while for instance the Schwartz derivative yields some terms which are ill-defined in this case. These contributions of the pseudo-function derivative actually take part in the values of $\hat{T}^{(\mathrm{CNC})}$ and $\hat{X}^{(\mathrm{CNC})}$ only. Denoting them by $\delta_{\text {self }} \hat{T}$ and $\delta_{\text {self }} \hat{X}$ in the case of the particular derivative (2.9) we find

$$
\begin{aligned}
\delta_{\text {self }} \hat{T} & =\frac{7}{12} \frac{G^{4} m_{1}^{3} m_{2}}{r_{1}^{2} r_{12}^{2}}\left(n_{1} n_{12}\right)+1 \leftrightarrow 2 \\
\delta_{\text {self }} \hat{X} & =\frac{G^{3} m_{1}^{3}}{c^{2} r_{1}^{3}}\left(-\frac{17}{72} \frac{G m_{2}}{r_{12}^{2}} r_{1}\left(n_{1} n_{12}\right)+\frac{1}{40}\left(n_{1} v_{1}\right)^{2}-\frac{1}{120} v_{1}^{2}\right)+1 \leftrightarrow 2
\end{aligned}
$$




\section{Interaction terms}

We consider exclusively the interaction terms (i) that are proportional to $m_{1}^{2} m_{2}$ rather than $m_{1} m_{2}^{2}$, (ii) that contribute at relative $1 \mathrm{PN}$ order, leaving aside those which are generated by $L_{i j}$. Depending on whether they come from "simple" or "composite" cubic parts as shown respectively below, the elementary terms composing the sources read schematically

$$
\begin{array}{ll} 
& \partial \operatorname{Pf}\left[F\left(\mathbf{r}_{1}\right)\right] \partial \operatorname{Pf}\left[G\left(\mathbf{r}_{1}\right)\right] \partial \operatorname{Pf}\left[H\left(\mathbf{r}_{2}\right)\right] \\
\text { and } \quad & \partial \operatorname{Pf}\left[F\left(\mathbf{r}_{1}\right)\right] \partial_{1} \partial_{2} \operatorname{Pf}\left[G\left(\mathbf{r}_{1}, \mathbf{r}_{2}\right)\right]=\partial_{2} \operatorname{Pf}\left\{\partial \operatorname{Pf}\left[F\left(\mathbf{r}_{1}\right)\right] \partial_{1} \operatorname{Pf}\left[G\left(\mathbf{r}_{1}, \mathbf{r}_{2}\right)\right]\right\} ;
\end{array}
$$

the functions $G, H$ belong to $\mathcal{F}$, and it is also the case of $F$ in general. However, there exist some composite terms for which $F=\ln r_{1}$, but this is not a trouble since $\partial F$ is still in $\mathcal{F}$. In the cases needed in this problem, $G$ is always one of the four functions: $g_{\text {match }}$,

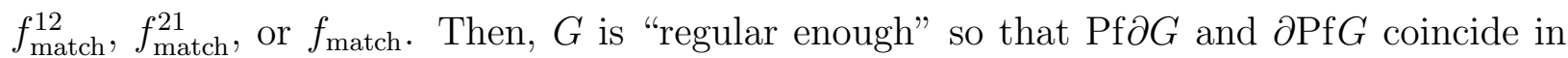
any cases, and further simplifications of the sources do not seem to be possible at this level. All we need, thus, is to transform the simple cubic contributions (5.8a) similarly to the self terms (see paragraph $\mathrm{VB})$. A typical example of elementary source we have to handle is $1 /\left(r_{1} r_{2}\right) \times \partial_{L}\left(1 / r_{1}\right)$, where $L$ represents at most two non-contracted indices. We can check that this term can be computed to Newtonian order only, hence it is given simply by a Poisson integral. In the language of pseudo-functions, this means that we have to evaluate:

$$
<\frac{1}{r_{1}^{\prime} r_{2}^{\prime}} \partial_{L}^{\prime} \operatorname{Pf} \frac{1}{r_{1}^{\prime}}, \frac{1}{-4 \pi\left|\mathbf{x}-\mathbf{x}^{\prime}\right|}>=<\frac{1}{r_{1}^{\prime}} \partial_{L}^{\prime} \operatorname{Pf} \frac{1}{r_{1}^{\prime}}, \frac{1}{-4 \pi\left|\mathbf{x}-\mathbf{x}^{\prime}\right| r_{2}^{\prime}}>
$$

The compact part of the dual bracket, which is associated with the distributional part of the derivative, when acting on $1 /\left(-4 \pi\left|\mathbf{x}-\mathbf{x}^{\prime}\right|\right)$, i.e.

$$
<\frac{1}{r_{1}^{\prime}} \mathrm{D}_{L}\left[\frac{1}{r_{1}^{\prime}}\right], \frac{1}{-4 \pi\left|\mathbf{x}-\mathbf{x}^{\prime}\right| r_{2}^{\prime}}>
$$

( $l=1$ or 2 ), leads a priori to a non-zero result. Here, $\mathrm{D}_{L}$ denotes the distributional part of the multi-derivative, obtained in Section VIII of 477 and recalled by the equation (2.12) above. However, the left side of the bracket, which is homogeneous to the $(-l-2)$ th power of a length, is necessarily of the type $r_{1}^{1-l} \operatorname{Pf} \delta_{1}$, times some dimensionless angular function whose multipolarity differs from $l$ by an even integer (because of the index structure of the operator $\left.\mathrm{D}_{L}\right)$. Now, the previous compact part is equal to the angular integral of the $r_{1}^{l-1}$ Taylor coefficient of $1 /\left(-4 \pi\left|\mathbf{x}-\mathbf{x}^{\prime}\right|\right)$, times the angular dependence of $\mathrm{D}_{L}\left[1 / r_{1}\right]$. The integrand then appears as a sum of terms whose multipolarity differs from $l+(l-1)=2 l-1$ by an even integer and so, is always odd; thereby the angular integral gives zero. By similar arguments, we can prove that the other compact sources associated with the distributional derivatives will never contribute to the Poisson integrals constituting the potentials we are considering here. Actually, it is possible to put together all the various kinds of simple-type [see (5.80)] cubic terms into a unique one, which is $n_{1}^{L} / r_{1}^{l+2} \times \partial \operatorname{Pf}\left(1 / r_{2}\right)$. We express at last the first factor (if $l \neq 0$ ) as a sum of derivatives of "scalars" thanks to identities such as 
(5.5). Since the pseudo-function derivatives will give here the same results as the Schwartz distributional ones, and by virtue of $\left.\partial_{L} \operatorname{Pf}\left(1 / r_{1}^{2}\right)\right|_{\mathcal{D}}=\left.\operatorname{Pf} \partial_{L}\left(1 / r_{1}^{2}\right)\right|_{\mathcal{D}}$ [where $\mathcal{D}$ is the set of smooth functions with compact support], the last transformation can be done in the sense of functions. Note however that the multiple derivatives of $1 / r_{2}$ are indeed distributional and play an important role in the sources.

To sum up what precedes, all the interaction terms have the general structure:

$$
\int \frac{d^{3} \mathbf{x}^{\prime}}{-4 \pi}\left|\mathbf{x}-\mathbf{x}^{\prime}\right|^{p} \partial_{2 L} \operatorname{Pf}\left[\partial_{J}^{\prime} \operatorname{Pf} F\left(\mathbf{r}_{1}^{\prime}\right) \partial_{1 K} \operatorname{Pf} G\left(\mathbf{r}_{1}^{\prime}, \mathbf{r}_{2}^{\prime}\right)\right]
$$

$(p=-1$ or $1, F$ and $G$ functions of $\mathcal{F}$ ). After commuting the integral and the derivative $\partial_{2 L}$, which is always licit for integrals converging at infinity (since $\partial_{2 L}$ is followed by a Pf), the general cubic term (5.9) becomes:

$$
\partial_{2 L} \int \frac{d^{3} \mathbf{x}^{\prime}}{-4 \pi}\left|\mathbf{x}-\mathbf{x}^{\prime}\right|^{p} \partial_{J}^{\prime} \operatorname{Pf} F\left(\mathbf{r}_{1}^{\prime}\right) \partial_{1 K} \operatorname{Pf} G\left(\mathbf{r}_{1}^{\prime}, \mathbf{r}_{2}^{\prime}\right) .
$$

In the case where $G=G\left(\mathbf{r}_{2}\right)$ (and $k=0$ ), this rearranges as

$$
\begin{aligned}
\partial_{2 L} \int \frac{d^{3} \mathbf{x}^{\prime}}{-4 \pi}\left|\mathbf{x}-\mathbf{x}^{\prime}\right|^{p}(-)^{j} \partial_{1 J}\left[\operatorname{Pf} F\left(\mathbf{r}_{1}^{\prime}\right) \operatorname{Pf} G\left(\mathbf{r}_{2}^{\prime}\right)\right] \\
=(-)^{j} \partial_{1 J} \partial_{2 L} \operatorname{Pf} \int \frac{d^{3} \mathbf{x}^{\prime}}{-4 \pi}\left|\mathbf{x}-\mathbf{x}^{\prime}\right|^{p} F\left(\mathbf{r}_{1}^{\prime}\right) G\left(\mathbf{r}_{2}^{\prime}\right)
\end{aligned}
$$

We shall end with implementing concretely our treatment of the source on two typical terms:

$$
\begin{aligned}
& \int \frac{d^{3} \mathbf{x}^{\prime}}{-4 \pi} \frac{1}{\left|\mathbf{x}-\mathbf{x}^{\prime}\right|} \frac{1}{r_{1}^{\prime}} \partial_{i}^{\prime} \operatorname{Pf} \frac{1}{r_{1}^{\prime}} \partial_{j}^{\prime} \operatorname{Pf} \frac{1}{r_{2}^{\prime}}=\frac{1}{2} \partial_{1 i} \partial_{2 j} \operatorname{Pf} \int \frac{d^{3} \mathbf{x}^{\prime}}{-4 \pi} \frac{1}{\left|\mathbf{x}-\mathbf{x}^{\prime}\right|} \frac{1}{r_{1}^{\prime 2} r_{2}^{\prime}} \\
& \int \frac{d^{3} \mathbf{x}^{\prime}}{-4 \pi} \frac{1}{\left|\mathbf{x}-\mathbf{x}^{\prime}\right|} k_{k i} f_{\text {match } j}^{12} \partial_{i j}^{\prime} \operatorname{Pf} \frac{1}{r_{1}^{\prime}}=\partial_{2 j} \int \frac{d^{3} \mathbf{x}^{\prime}}{-4 \pi} \frac{1}{\left|\mathbf{x}-\mathbf{x}^{\prime}\right|}{ }_{k i} f_{\text {match }}^{12} \partial_{i j}^{\prime} \operatorname{Pf} \frac{1}{r_{1}^{\prime}}
\end{aligned}
$$

and by providing the complete interaction component corresponding to the Poisson integral of $K_{i j} \partial_{i j} U$, which completes the self part obtained previously in (5.6). The compact support terms have been explicitly determined, while the other ones are left un-evaluated for the moment:

$$
\begin{gathered}
\left(\int \frac{d^{3} \mathbf{x}^{\prime}}{-4 \pi} \frac{1}{\left|\mathbf{x}-\mathbf{x}^{\prime}\right|} K_{i j} \partial_{i j} U\right)_{\mathrm{int}}=G^{3} m_{1}^{2} m_{2}\left\{\frac{a_{1}^{i}}{4} \partial_{2 i} D \operatorname{Pf} \int \frac{d^{3} \mathbf{x}^{\prime}}{-4 \pi} \frac{1}{\left|\mathbf{x}-\mathbf{x}^{\prime}\right|} \frac{\ln r_{1}^{\prime}}{r_{2}^{\prime}}\right. \\
+\frac{a_{1}^{i}}{48} \partial_{1 i} D^{2} \operatorname{Pf} \int \frac{d^{3} \mathbf{x}^{\prime}}{-4 \pi} \frac{1}{\left|\mathbf{x}-\mathbf{x}^{\prime}\right|} \frac{r_{1}^{\prime 2} \ln r_{1}^{\prime}}{r_{2}^{\prime}}+a_{1}^{j} \partial_{2 i} \operatorname{Pf} \int \frac{d^{3} \mathbf{x}^{\prime}}{-4 \pi} \frac{1}{\left|\mathbf{x}-\mathbf{x}^{\prime}\right|}{ }_{j k} f_{\text {match }}^{12} \partial_{i k}^{\prime} \frac{1}{r_{1}^{\prime}} \\
+a_{2}^{j} \partial_{2 i j} \operatorname{Pf} \int \frac{d^{3} \mathbf{x}^{\prime}}{-4 \pi} \frac{1}{\left|\mathbf{x}-\mathbf{x}^{\prime}\right|} k f_{\text {match }}^{21} \partial_{i k}^{\prime} \frac{1}{r_{1}^{\prime}}-\frac{\left(n_{12} a_{1}\right)}{8 r_{12} r_{2}} \\
+\frac{v_{1}^{i} v_{1}^{j}}{8} \partial_{2 i j} \operatorname{Pf} \int \frac{d^{3} \mathbf{x}^{\prime}}{-4 \pi} \frac{1}{\left|\mathbf{x}-\mathbf{x}^{\prime}\right|} \frac{1}{r_{1}^{\prime 2} r_{2}^{\prime}}+\frac{v_{1}^{2}}{16} D^{2} \operatorname{Pf} \int \frac{d^{3} \mathbf{x}^{\prime}}{-4 \pi} \frac{1}{\left|\mathbf{x}-\mathbf{x}^{\prime}\right|} \frac{\ln r_{1}^{\prime}}{r_{2}^{\prime}}
\end{gathered}
$$




$$
\begin{aligned}
& +\frac{v_{1}^{i} v_{1}^{j}}{96} \partial_{1 i j} D^{2} \operatorname{Pf} \int \frac{d^{3} \mathbf{x}^{\prime}}{-4 \pi} \frac{1}{\left|\mathbf{x}-\mathbf{x}^{\prime}\right|} \frac{r_{1}^{\prime 2} \ln r_{1}^{\prime}}{r_{2}^{\prime}} \\
& +v_{1}^{j} v_{1}^{k} \partial_{2 i} \operatorname{Pf} \int \frac{d^{3} \mathbf{x}^{\prime}}{-4 \pi} \frac{1}{\left|\mathbf{x}-\mathbf{x}^{\prime}\right|} j_{k l} f_{\text {match }}^{12} \partial_{i l}^{\prime} \frac{1}{r_{1}^{\prime}} \\
& \left.+v_{2}^{j} v_{2}^{k} \partial_{2 i j k} \operatorname{Pf} \int \frac{d^{3} \mathbf{x}^{\prime}}{-4 \pi} \frac{1}{\left|\mathbf{x}-\mathbf{x}^{\prime}\right|} l f_{\text {match }}^{21} \partial_{i l}^{\prime} \frac{1}{r_{1}^{\prime}}+\frac{\left(n_{12} v_{1}\right)^{2}}{8 r_{12}^{2} r_{2}}\right\}+1 \leftrightarrow 2+\mathcal{O}(1),
\end{aligned}
$$

where $D$ denotes the operator $\partial_{1 i} \partial_{2 i}$.

\section{Elementary integrals}

\section{Nomenclature}

The inspection of the formula (5.12) for interaction terms issued from $K_{i j} \partial_{i j} U$ suggests that we should re-express this potential, as well as all the other ones, by means of a restricted number of elementary integrals; basically one for each source type. Hence the proposal for a useful systematic nomenclature, which reflects their structure. We shall introduce the following notations (and ditto $1 \leftrightarrow 2$ ):

$$
\begin{aligned}
& \stackrel{(n, p)}{\mathcal{Y}}=\operatorname{Pf} \int \frac{d^{3} \mathbf{x}^{\prime}}{-4 \pi} \frac{1}{\left|\mathbf{x}-\mathbf{x}^{\prime}\right|} r_{1}^{\prime n} r_{2}^{\prime p} \quad \mathcal{F}_{1(P, Q)}^{12}=\int \frac{d^{3} \mathbf{x}^{\prime}}{-4 \pi} \frac{1}{\left|\mathbf{x}-\mathbf{x}^{\prime}\right|}{ }_{i Q} f_{\text {match }}^{12} \partial_{i P}^{\prime} \operatorname{Pf} \frac{1}{r_{1}^{\prime}} \\
& \stackrel{(n, p)}{\mathcal{N}}=\operatorname{Pf} \int \frac{d^{3} \mathbf{x}^{\prime}}{-4 \pi} \frac{1}{\left|\mathbf{x}-\mathbf{x}^{\prime}\right|} r_{1}^{\prime n} r_{2}^{\prime p} \ln r_{1}^{\prime} \quad \mathcal{F}_{1(P, Q)}^{21}=\int \frac{d^{3} \mathbf{x}^{\prime}}{-4 \pi} \frac{1}{\left|\mathbf{x}-\mathbf{x}^{\prime}\right|} i_{Q} f_{\text {match }}^{21} \partial_{i P}^{\prime} \operatorname{Pf} \frac{1}{r_{1}^{\prime}} \\
& \mathcal{U}_{P}=\int \frac{d^{3} \mathbf{x}^{\prime}}{-4 \pi} \frac{1}{\left|\mathbf{x}-\mathbf{x}^{\prime}\right|}{ }_{k} g_{\text {match k }} \partial_{P}^{\prime} \operatorname{Pf} \frac{1}{r_{1}^{\prime}} \quad{\underset{1}{\mathcal{F}}}_{(P, Q)}=\int \frac{d^{3} \mathbf{x}^{\prime}}{-4 \pi} \frac{1}{\left|\mathbf{x}-\mathbf{x}^{\prime}\right|}{ }_{i Q} f_{\text {match }} \partial_{i P}^{\prime} \operatorname{Pf} \frac{1}{r_{1}^{\prime}} \\
& \underset{1_{P}}{\mathcal{K}_{P}}=\int \frac{d^{3} \mathbf{x}^{\prime}}{-4 \pi} \frac{1}{\left|\mathbf{x}-\mathbf{x}^{\prime}\right|} g_{\text {match }} \partial_{P} \operatorname{Pf} \frac{1}{r_{1}^{\prime}} \quad \stackrel{(n, p)}{\mathcal{S}}=\operatorname{Pf} \int \frac{d^{3} \mathbf{x}^{\prime}}{-4 \pi}\left|\mathbf{x}-\mathbf{x}^{\prime}\right| r_{1}^{\prime n} r_{2}^{\prime p}
\end{aligned}
$$

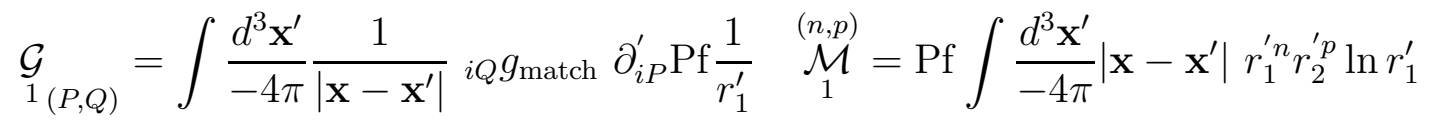

$$
\begin{aligned}
& { }_{1}^{\mathcal{G}_{(P, Q)}}=\int \frac{d^{3} \mathbf{x}^{\prime}}{-4 \pi} \frac{1}{\left|\mathbf{x}-\mathbf{x}^{\prime}\right|} i_{Q} g_{\text {match }} \partial_{P}^{\prime} \operatorname{Pf} \frac{1}{r_{1}^{\prime}} \underset{1_{(P, Q)}}{\mathcal{Q}}=\int \frac{d^{3} \mathbf{x}^{\prime}}{-4 \pi}\left|\mathbf{x}-\mathbf{x}^{\prime}\right|_{i Q} g_{\text {match }} \partial_{i P}^{\prime} \operatorname{Pf} \frac{1}{r_{1}^{\prime}} \\
& {\underset{1}{\mathcal{H}}}_{(P, Q)}=\int \frac{d^{3} \mathbf{x}^{\prime}}{-4 \pi} \frac{1}{\left|\mathbf{x}-\mathbf{x}^{\prime}\right|}{ }_{i Q} g_{\text {match }} \partial_{i P}^{\prime} \operatorname{Pf} r_{1}^{\prime} \text {. }
\end{aligned}
$$

The value of the previous integrals is not generally known at any space location, except in some special cases. The reason is that their sources involve three points, in addition to the integration variable $\mathbf{x}^{\prime}$ : the point $\mathbf{x}$ where the field is calculated, and the two source points $\mathbf{y}_{1}$, $\mathbf{y}_{2}$. The few ones that are computable for any $\mathbf{x}$ include notably some self integrals like $\mathcal{Y}^{(n, 0)}$ and $\mathcal{S}^{(n, 0)}$, and the two integrals $D^{2} \mathcal{N}_{1}^{(0,-1)}$ and $\partial_{2 i} \mathcal{G}_{1(i, 0)}$ entering the interaction part of the $\hat{X}^{(\mathrm{CNC})}$ potential at the Newtonian order. Actually, there are no other cubic contributions up to the 2.5PN order, and that is why we were able in [31] to get the complete expression of the metric in the near zone at this order. This property of the integrals $D^{2} \mathcal{N}_{1}^{(0,-1)}$ and $\partial_{2 i} \mathcal{G}_{1(i, 0)}$ 
is linked to the specific form of the integrands, which are made of products of two second derivatives applied on appropriate functions, such that the indices of the first derivative are contracted with those of the second one: $\partial_{i j} \operatorname{Pf} \ln r_{1} \times \partial_{i j} \operatorname{Pf}\left(1 / r_{2}\right)$ and ${ }_{i} g_{j} \times \partial_{i j} \operatorname{Pf}\left(1 / r_{1}\right)$. In both cases, particular solutions in the sense of distributions of the corresponding Poisson equations

$$
\Delta K_{1}=2 \partial_{i j} \operatorname{Pf} \ln r_{1} \partial_{i j} \mathrm{Pf} \frac{1}{r_{2}}, \quad \Delta H_{1}=2_{i} g_{j} \partial_{i j} \mathrm{Pf} \frac{1}{r_{1}} .
$$

can be exhibited [57.31. The solutions $K_{1}$ and $H_{1}$ of (5.14) that go to zero as $r \rightarrow \infty$ read:

$$
\begin{aligned}
K_{1} & =\left(\frac{1}{2} \Delta-\Delta_{1}\right)\left[\frac{\ln r_{1}}{r_{2}}\right]+\frac{1}{2} \Delta_{2}\left[\frac{\ln r_{12}}{r_{2}}\right]+\frac{r_{2}}{2 r_{12}^{2} r_{1}^{2}}+\frac{1}{r_{12}^{2} r_{2}}, \\
H_{1} & =\frac{1}{2} \Delta_{1}\left[\frac{g}{r_{1}}+\frac{\ln r_{1}}{r_{12}}-\Delta_{1}\left(\frac{r_{1}+r_{12}}{2} g\right)\right] \\
& +\partial_{i} \partial_{2 i}\left[\frac{\ln r_{12}}{r_{1}}+\frac{\ln r_{1}}{2 r_{12}}\right]-\frac{1}{r_{1}} \partial_{2 i}\left[\left(\partial_{i} g\right)_{1}\right]-\frac{r_{2}}{2 r_{1}^{2} r_{12}^{2}},
\end{aligned}
$$

with $\Delta_{1}=\partial_{1 i i}, \Delta_{2}=\partial_{2 i i}$; see also (5.32) for the expanded forms of these solutions. Thus, we have

$$
D^{2} \mathcal{N}_{1}^{(0,-1)}=\frac{1}{2} K_{1} \quad \text { and } \quad \partial_{2 i} \mathcal{G}_{1(i, 0)}=\frac{1}{2} H_{1} .
$$

In the list (5.13) above, we note the appearance of iterated Poisson integrals such as $\mathcal{S}^{(-5,0)}$, $\mathcal{M}^{(0,-1)}$ and $\partial_{2 i} \mathcal{Q}_{1(i, 0)}$ which come from the $1 \mathrm{PN}$ contribution to the retardation expansion of $\hat{X}^{(\mathrm{CNC})}$, and thus enter this potential through their second time derivative. What we shall have to compute for our purpose is their Hadamard regularized value. However, $\partial_{2 i} \mathcal{Q}_{1(i, 0)}$ is not available on the whole space (for any $\mathbf{x}$ ), so that we cannot deduce $\partial_{t}^{2} \partial_{2 i} \mathcal{Q}_{1(i, 0)}$ from it directly. We shall adopt then a different approach. In a first stage, we express the operator $\partial_{t}^{2}$ with the help of the partial derivatives $\partial_{1 i}$ and $\partial_{2 i}$ : if $F\left(\mathbf{r}_{1}, \mathbf{r}_{2}\right)=\partial_{2 i} \mathcal{Q}_{1(i, 0)}$, then

$$
\partial_{t}^{2} F=\partial_{t}\left[v_{1}^{i} \partial_{1 i} F\right]=v_{1}^{i} v_{1}^{j} \partial_{1 i j} F+a_{1}^{i} \partial_{1 i} F+1 \leftrightarrow 2 .
$$

Next, we commute the partial derivatives $\partial_{1 L}$ with the integration sign, so that the derivatives act on the source of the Poisson integral. This operation is legitimate only if the derivatives $\partial_{1 L}$ when acting on the integrands are viewed as distributional (in the sense of Section IX in [47]). The new integrands write then as a partie finie derivative of a product, e.g. $\partial_{1 L}\left({ }_{i Q} g \partial_{i P}^{\prime} \frac{1}{r_{1}^{\prime}}\right)$; but remember that we are not a priori allowed to develop them according to the Leibniz rule in our formalism. In fact, these specific non Leibniz corrections happen to give zero contribution to the 3PN potentials. This can be seen by applying successively the formula (7.23) in [47] (which is indeed sufficient since the "test" function $\left|\mathbf{x}-\mathbf{x}^{\prime}\right|$ is smooth at the points 1 and 2) to all the sources we are dealing with. Therefore, we can employ the usual rule for derivatives of products to perform our final transformation. In summary, we will have for instance 


$$
\begin{aligned}
\partial_{1 k l} \int \frac{d^{3} \mathbf{x}^{\prime}}{-4 \pi} \mid \mathbf{x}- & \left.\mathbf{x}^{\prime}\right|_{i} g_{j} \partial_{i j} \operatorname{Pf} \frac{1}{r_{1}^{\prime}}=\int \frac{d^{3} \mathbf{x}^{\prime}}{-4 \pi}\left|\mathbf{x}-\mathbf{x}^{\prime}\right|_{i k l} g_{j} \partial_{i j} \operatorname{Pf} \frac{1}{r_{1}^{\prime}} \\
& +2 \int \frac{d^{3} \mathbf{x}^{\prime}}{-4 \pi}\left|\mathbf{x}-\mathbf{x}^{\prime}\right|_{i(k} g_{\underline{j}} \partial_{l) i j} \operatorname{Pf} \frac{1}{r_{1}^{\prime}}+\int \frac{d^{3} \mathbf{x}^{\prime}}{-4 \pi}\left|\mathbf{x}-\mathbf{x}^{\prime}\right|_{i} g_{j} \partial_{i j k l} \operatorname{Pf} \frac{1}{r_{1}^{\prime}}
\end{aligned}
$$

(the index $\underline{j}$ means that $j$ is excluded from the symmetrization operation). On this form we can apply the partie finie at 1 while staying in the same class of elementary integrals (5.13). We conclude by going back to the example of the cubic term generated by $K_{i j} \partial_{i j} U$; we have, after appropriate reshaping,

$$
\begin{aligned}
& \int \frac{d^{3} \mathbf{x}^{\prime}}{-4 \pi} \frac{1}{\left|\mathbf{x}-\mathbf{x}^{\prime}\right|} K_{i j} \partial_{i j} U=G^{3} m_{1}^{3}\left\{-\frac{a_{1}^{i}}{6} \partial_{1 i}^{(-3,0)} \mathcal{Y}+\frac{v_{1}^{i} v_{1}^{j}}{30} \partial_{1 i j}^{(-3,0)} \mathcal{Y}^{\left(2 v_{1}^{2}\right.} \stackrel{(-5,0)}{\mathcal{Y}}^{(-2}\right. \\
& \left.-\frac{\left(n_{1} a_{1}\right)}{12 r_{1}^{2}}+\frac{7\left(n_{1} v_{1}\right)^{2}}{150 r_{1}^{3}}-\frac{7 v_{1}^{2}}{450 r_{1}^{3}}\right\} \\
& +G^{3} m_{1}^{2} m_{2}\left\{\frac{a_{1}^{i}}{4} \partial_{2 i} D \stackrel{(0,-1)}{\mathcal{N}}+\frac{a_{1}^{i}}{48} \partial_{1 i} D^{2} \stackrel{(2,-1)}{\mathcal{N}}_{1}^{\left(a_{1}\right.} a_{1}^{j} \partial_{1(i, j)} \mathcal{F}^{12}+a_{2}^{j} \partial_{2 i j} \mathcal{F}_{(i, 0)}^{21}\right. \\
& -\frac{\left(n_{12} a_{1}\right)}{8 r_{12} r_{2}}+\frac{v_{1}^{i} v_{1}^{j}}{8} \partial_{2 i j}^{(-2,-1)} \mathcal{Y}+\frac{v_{1}^{2}}{16} D^{2} \stackrel{(0,-1)}{\mathcal{N}}_{1}^{\left(v_{1}^{i} v_{1}^{j}\right.} \partial_{1 i j} D^{2} \stackrel{(2,-1)}{\mathcal{N}}_{1} \\
& \left.+v_{1}^{j} v_{1}^{k} \partial_{2 i} \mathcal{F}_{(i, j k)}^{12}+v_{2}^{j} v_{2}^{k} \partial_{2 i j k} \mathcal{F}_{(i, 0)}^{21}+\frac{\left(n_{12} v_{1}\right)^{2}}{8 r_{12}^{2} r_{2}}\right\}+1 \leftrightarrow 2+\mathcal{O}(1) .
\end{aligned}
$$

We refer to [52] for the expressions of the other non-linear potentials expressed in this manner by means of the same nomenclature. The problem is now to evaluate all the elementary integrals from which the $3 \mathrm{PN}$ cubic potentials have been built.

\section{Parties finies of the elementary integrals}

As mentioned before, in most cases, we do not have at our disposal the explicit values of the elementary integrals in all space. This does not matter since all we need is their Hadamard partie finie at point 1 (or 2). Notice that the partial derivative with respect to $\mathbf{y}_{2}$ is the only one that commutes with the partie finie operation at 1 ; to be more explicit:

$$
\begin{array}{ll} 
& {\left[\partial_{2 i} F\left(\mathbf{x}, \mathbf{y}_{1}, \mathbf{y}_{2}\right)\right]_{1}=\partial_{2 i}\left[F\left(\mathbf{x}, \mathbf{y}_{1}, \mathbf{y}_{2}\right)\right]_{1},} \\
\text { but } \quad\left[\partial_{1 i} F\left(\mathbf{x}, \mathbf{y}_{1}, \mathbf{y}_{2}\right)\right]_{1} \neq \partial_{1 i}\left[F\left(\mathbf{x}, \mathbf{y}_{1}, \mathbf{y}_{2}\right)\right]_{1} \quad(\text { with } F, G \in \mathcal{F}) .
\end{array}
$$

Thus, for each elementary integral, we shall determine first the partie finie of the quantity figuring under the derivation symbol $\partial_{2}$ and, only then, apply the latter operator. On the contrary, we cannot bring the derivatives with respect to $\mathbf{y}_{1}$ out of the partie finie at 1 , so we are led to incorporate them to the sources, in the sense of pseudo-functions, by permutation with the integration sign. As a consequence, the integrals we are interested in are of the type:

$$
\int \frac{d^{3} \mathbf{x}}{-4 \pi}\left|\mathbf{x}-\mathbf{x}^{\prime}\right|^{p} \partial_{1}\left[\operatorname{Pf} F\left(\mathbf{r}_{1}\right)\right] \partial_{1}\left[\operatorname{Pf} G\left(\mathbf{r}_{1}, \mathbf{r}_{2}\right)\right]
$$




$$
=-\frac{1}{4 \pi}<\partial_{1}\left[\operatorname{Pf} F\left(\mathbf{r}_{1}\right)\right] \partial_{1}\left[\operatorname{Pf} G\left(\mathbf{r}_{1}, \mathbf{r}_{2}\right)\right],\left|\mathbf{x}-\mathbf{x}^{\prime}\right|^{p}>
$$

They involve both a compact part (C) and a non-compact part (NC). The compact part is produced by the purely distributional contributions of derivatives in the integrands:

$-\frac{1}{4 \pi}<\mathrm{D}_{1}\left[F\left(\mathbf{r}_{1}\right)\right] \operatorname{Pf} \partial_{1} G\left(\mathbf{r}_{1}, \mathbf{r}_{2}\right),\left|\mathbf{x}-\mathbf{x}^{\prime}\right|^{p}>-\frac{1}{4 \pi}<\mathrm{D}_{1}\left[G\left(\mathbf{r}_{1}, \mathbf{r}_{2}\right)\right] \operatorname{Pf} \partial_{1} F\left(\mathbf{r}_{1}\right),\left|\mathbf{x}-\mathbf{x}^{\prime}\right|^{p}>$

As mentioned at the beginning of the paragraph $\overline{\mathrm{VQ}}$, the partie finie derivatives reduce here, in our case, to those of the Schwartz theory. This is obvious when $G=G\left(\mathbf{r}_{2}\right)$, because then the source $F\left(\mathbf{r}_{1}\right)$, regarded as a linear functional $\operatorname{Pf} F\left(\mathbf{r}_{1}\right)$, acts on a function which is smooth in a neighbourhood of $\mathbf{x}=\mathbf{y}_{1}$; in the other cases, the result follows from explicit calculations. Note that the number $l$ of derivatives in front of $G\left(\mathbf{r}_{1}, \mathbf{r}_{2}\right)=g_{\text {match }}$, $f_{\text {match }}^{12}, f_{\text {match }}^{21}$, or $f_{\text {match }}$ is always small enough so that $\partial_{1 L-1} G\left(\mathbf{r}_{1}, \mathbf{r}_{2}\right)$ is bounded, hence $\partial_{1 L} \operatorname{Pf} G\left(\mathbf{r}_{1}, \mathbf{r}_{2}\right)=\operatorname{Pf} \partial_{1 L} G\left(\mathbf{r}_{1}, \mathbf{r}_{2}\right)$. Once we have in hands the compact part, it remains to obtain its regularized value at point 1 . As a matter of fact, if the source is of the type $\operatorname{Pf} F \delta_{2}$, where $F \in \mathcal{F}$, then

$$
<\operatorname{Pf}\left(F \delta_{2}\right)\left(\mathbf{x}^{\prime}\right), \frac{1}{\left|\mathbf{x}-\mathbf{x}^{\prime}\right|}>=-\frac{1}{4 \pi} \sum_{l \geq 0} \frac{(-)^{l}}{l !}\left(r_{2}^{\prime l} n_{2}^{\prime L} F\right)_{2} \partial_{L}\left(\frac{1}{r_{2}}\right)
$$

is smooth at point 1 and we need not call for the Hadamard regularization. Therefore, we are allowed to replace directly $\left|\mathbf{x}-\mathbf{x}^{\prime}\right|$ by $r_{1}^{\prime}$ in the left-hand-side of (5.16). When the source is of the generic type $\operatorname{Pf} F \delta_{1}$, the same identity as (5.16) holds, but with $2 \rightarrow 1$. This shows that the integral $<\operatorname{Pf}\left(F \delta_{1}\right),\left|\mathbf{x}-\mathbf{x}^{\prime}\right|^{-1}>$ is purely singular as $r_{1} \rightarrow 0$, which means that it has no partie finie at $\mathbf{x}=\mathbf{y}_{1}$ : the $\delta_{1}$ type sources do not contribute to the potentials computed at body 1. Summarizing, we have

$$
\begin{aligned}
& \left(<\operatorname{Pf}\left(F \delta_{2}\right), \frac{1}{\left|\mathbf{x}-\mathbf{x}^{\prime}\right|}>\right)_{1}=<\operatorname{Pf}\left(F \delta_{2}\right), \frac{1}{r_{1}}> \\
& \left(<\operatorname{Pf}\left(F \delta_{1}\right), \frac{1}{\left|\mathbf{x}-\mathbf{x}^{\prime}\right|}>\right)_{1}=0
\end{aligned}
$$

We refer the reader to equations (6.17)-(6.20) in 477 for more details.

Let us now focus our attention on the non-compact parts of the elementary integrals, whose integrands are made, by definition, of ordinary functions. The problem is to get the Hadamard partie finie of the Poisson integral $P$ of $F \in \mathcal{F}$ :

$$
P\left(\mathbf{x}^{\prime}\right)=\operatorname{Pf} \int \frac{d^{3} \mathbf{x}}{-4 \pi} \frac{1}{\left|\mathbf{x}-\mathbf{x}^{\prime}\right|} F(\mathbf{x})
$$

as well as the one of the iterated Poisson integral $Q$, given by:

$$
Q\left(\mathbf{x}^{\prime}\right)=\operatorname{Pf} \int \frac{d^{3} \mathbf{x}}{-4 \pi}\left|\mathbf{x}-\mathbf{x}^{\prime}\right| F(\mathbf{x}) \text {. }
$$


[Each of these functions depends also on the source points $\mathbf{y}_{1,2}$ and velocities $\mathbf{v}_{1,2}$.] Now, the partie finie prescription applies only to functions admitting power-like expansions near their singularities [see (2.1)], whereas $P$ or $Q$ may contain logarithmic coefficients in their development: if we take for instance $F=1 / r_{1}^{3}$, we shall have $P=-\left[1+\ln \left(r_{1}^{\prime} / s_{1}\right)\right] / r_{1}^{\prime}$, where $s_{1}$ is the constant appearing in (2.3). Following [47], we shall simply include the possible logarithms (i.e. $\ln r_{1}^{\prime}$ ) appearing in the zeroth power coefficient of the expansion of $P$ or $Q$ in the definition of the partie finie: see the equation (5.4) in [47. With this generalized notion of "partie finie", we can give a sense to $(P)_{1}$ and $(Q)_{1}$, as well as their gradients $\left(\partial_{i} P\right)_{1}$ and $\left(\partial_{i} Q\right)_{1}$. We make then the following statements (see Section $\mathrm{V}$ of [47] for proofs and discussion):

$$
\begin{aligned}
(P)_{1} & =\operatorname{Pf}_{s_{1}, s_{2}} \int \frac{d^{3} \mathbf{x}}{-4 \pi} \frac{1}{r_{1}} F+\left[\ln \left(\frac{r_{1}^{\prime}}{s_{1}}\right)-1\right]\left(r_{1}^{2} F\right)_{1}, \\
(Q)_{1} & =\operatorname{Pf}_{s_{1}, s_{2}} \int \frac{d^{3} \mathbf{x}}{-4 \pi} r_{1} F+\left[\ln \left(\frac{r_{1}^{\prime}}{s_{1}}\right)+\frac{1}{2}\right]\left(r_{1}^{4} F\right)_{1}, \\
\left(\partial_{i} P\right)_{1} & =\operatorname{Pf}_{s_{1}, s_{2}} \int \frac{d^{3} \mathbf{x}}{-4 \pi} \frac{n_{1}^{i}}{r_{1}^{2}} F+\ln \left(\frac{r_{1}^{\prime}}{s_{1}}\right)\left(n_{1}^{i} r_{1} F\right)_{1}, \\
\left(\partial_{i} Q\right)_{1} & =\operatorname{Pf}_{s_{1}, s_{2}} \int \frac{d^{3} \mathbf{x}}{4 \pi} n_{1}^{i} F+\left[-\ln \left(\frac{r_{1}^{\prime}}{s_{1}}\right)+\frac{1}{2}\right]\left(n_{1}^{i} r_{1}^{3} F\right)_{1},
\end{aligned}
$$

where the first terms in the right sides are made of some partie-finie integrals in the sense of the definition (2.3). The "constant" $r_{1}^{\prime}$ is the variable which tends toward zero when evaluating the partie finie. It is easy to show that the constant $s_{1}$ cancels out between the two terms in each of the second members of the identities (5.18). Indeed, using the general dependence of the partie-finie integral on the constants $s_{1}, s_{2}$ as given by (4.20) in [47], we easily see that $(P)_{1}$ given by (5.18a), for instance, depends on the constants $r_{1}^{\prime}$ and $s_{2}$ through the formula

$$
(P)_{1}=-\ln \left(\frac{r_{12}}{r_{1}^{\prime}}\right)\left(r_{1}^{2} F\right)_{1}-\ln \left(\frac{r_{12}}{s_{2}}\right)\left(\frac{r_{2}^{3}}{r_{1}} F\right)_{2}+\ldots
$$

where the dots indicate the terms that are independent of any constants. As we see, the constant $s_{1}$ has been so to speak "replaced" by $r_{1}^{\prime}$. This makes clearer why it is convenient to keep the $\ln r_{1}^{\prime}$ in the definition of the Hadamard partie finie; if we had decided to exclude this logarithm from it, we would have found some bare $\ln r_{12}$ in the first term of (5.19) instead of a nicer logarithm of a dimensionless quantity: $\ln \left(r_{12} / r_{1}^{\prime}\right)$; but this is simply a matter of convenience, because we shall see that in fact the "constants" $\ln r_{1}^{\prime}$ and $\ln r_{2}^{\prime}$ can be gauged away from the 3PN equations of motion. The same argument is valid for all cases in (5.18). As a consequence, the acceleration of the first body will depend only on two unspecified constants: $\ln r_{1}^{\prime}$, and of course $\ln s_{2}$ (and ditto for the acceleration of the second body). See Section VII for further discussion of these constants.

The relations (5.18) answer the problem of evaluating the elementary integrals at the location of particle 1 without knowing their values at an arbitrary field point. The subsequent 
task consists of calculating the partie-finie integrals in the right-hand-sides, which will turn out to be always possible.

\section{Integration methods}

The non compact parts of the regularized elementary integrals consist essentially of some integrals $\operatorname{Pf} \int d^{3} \mathbf{x} F\left(\mathbf{r}_{1}, \mathbf{r}_{2}\right)$, with $F \in \mathcal{F}$. It is worth noting that the sources depend on $\mathbf{r}_{1}$, $\mathbf{r}_{2}$ exclusively, and not on the separate variables $\mathbf{x}, \mathbf{y}_{1}$, and $\mathbf{y}_{2}$, because scalar products like $\left(x y_{1}\right),\left(x y_{2}\right)$ or $\left(y_{1} y_{2}\right)$ occurring in $f_{\text {match }}^{12}, f_{\text {match }}^{21}$, and $f_{\text {match }}$ are killed by the derivatives that precede them in the integrand. In this work, we make use of two different integration methods: (1) the angular method, in which we determine successively angular and radial integrals in spherical coordinates, (2) the analytic continuation method, based on the socalled Riesz formula.

a. Angular method. Let $F=F\left(\mathbf{r}_{1}, \mathbf{r}_{2}\right)$ be a function in the class $\mathcal{F}$. We assume for a moment that $F$ is locally integrable at point 2 ; so we are allowed to compute the integral over the whole space, deprived from a small ball $\mathcal{B}_{1}(s)$ of center $\mathbf{y}_{1}$ and radius $s>0$. Thus, we start with the well-defined quantity $\int_{\mathbb{R}^{3} \backslash \mathcal{B}_{1}(s)} d^{3} \mathbf{x} F\left(\mathbf{r}_{1}, \mathbf{r}_{2}\right)$, but for convenience we write it in spherical coordinates $\left(r_{1}, \theta_{1}, \phi_{1}\right)$, such that the azimuthal angle $\theta_{1}$ coincides with the separation angle between $\mathbf{y}_{12}$ and $\mathbf{r}_{1}$,

$$
\int_{\mathbb{R}^{3} \backslash \mathcal{B}_{1}(s)} d^{3} \mathbf{x} F\left(\mathbf{r}_{1}, \mathbf{r}_{2}\right)=\int_{r_{1}}^{+\infty} d r_{1} r_{1}^{2} \int d \Omega_{1} F\left(\mathbf{r}_{1}, \mathbf{r}_{2}\right) .
$$

Actually the function $F$ may be a tensor with many indices, but the only unit vectors in the problem are $\mathbf{n}_{1}, \mathbf{n}_{2}, \mathbf{n}_{12}$, and only two of them, say $\mathbf{n}_{1}$ and $\mathbf{n}_{12}$, are independent, by virtue of the relation $r_{1} \mathbf{n}_{1}+r_{12} \mathbf{n}_{12}=r_{2} \mathbf{n}_{2}$; and therefore, $F$ can be expressed under the form of a finite sum of tensorial products of type $n_{1}^{L} n_{12}^{K}(l, k \in \mathbb{N})$; moreover, each factor admits a symmetric trace-free (STF) decomposition on the basis $\hat{n}_{1}^{L}=\mathrm{STF} n_{1}^{L}$. Hence we have:

$$
F=\sum_{l=0}^{l_{0}} \sum_{k=0}^{k_{0}} \hat{n}_{1}^{L} n_{12}^{K} G_{(l k)}\left(r_{1}, r_{2}\right), \quad l_{0}, k_{0} \in \mathbb{N} .
$$

Here, the $G_{(l k)}$ 's are some scalar functions of $r_{1}$ and $r_{2}$. Now, $r_{2}$ is related to $r_{1}$ and the scalar product $\left(n_{1} n_{12}\right)$ by $r_{2}=\sqrt{r_{1}^{2}+r_{12}^{2}+2 r_{1} r_{12}\left(n_{1} n_{12}\right)}$. So that the angular integral of $F$ can be obtained by means of the "mean formula"; see, e.g., the formula (A26) in [54]. We get

$$
\int d \Omega_{1} F=2 \pi \sum_{l=0}^{l_{0}} \sum_{k=0}^{k_{0}} \hat{n}_{12}^{L} n_{12}^{K} \int_{-1}^{1} d z G_{(l k)}\left(r_{1}, \sqrt{r_{1}^{2}+r_{12}^{2}+2 r_{1} r_{12} z}\right) P_{l}(z)
$$

where $P_{l}$ is the Legendre polynomial of order $l$. In the cases of interest here, $G_{(l k)}$ is always a sum of rational fractions with general structure $r_{1}^{p} r_{2}^{q} /\left(r_{1}+r_{2}+r_{12}\right)^{n}$, with $p, q$ positive or 
negative integers and $n \in \mathbb{N}$. It is easy to check that the result of the angular integration depends on the relative positions of $r_{1}$ and $r_{12}$. Therefore we must split the radial integral into two contributions according to the integration domains $\left.\left.r_{1} \in\right] 0, r_{12}\right]$ or $\left.r_{1} \in\right] r_{12},+\infty[$. Typical terms coming from the angular integration are $r_{1}^{p} /\left(r_{1}+r_{12}\right)^{n}$, as well as some more complicated logarithmic terms such as $r_{1}^{p} \ln \left(1+r_{12} / r_{1}\right)$. Most of the corresponding radial integrals are obtained straightforwardly using some integrations by part, applying the partie finie at the bound $r_{1}=s$ [i.e., removing the poles $1 / s^{k}$, with $k \geq 1$, and replacing $\ln s$ by $\left.\ln s_{1}\right]$. In the case of the latter logarithmic terms with $p=-1$, integrating by parts does not lead to anything, but the radial integrals can be found in standard mathematical textbooks:

$$
\begin{aligned}
& \int_{r_{12}}^{+\infty} \frac{d r_{1}}{r_{1}} \ln \left(1+\frac{r_{12}}{r_{1}}\right)=\int_{0}^{r_{12}} \frac{d r_{1}}{r_{1}} \ln \left(1+\frac{r_{1}}{r_{12}}\right)=\frac{\pi^{2}}{12} \\
& \int_{r_{12}}^{+\infty} \frac{d r_{1}}{r_{1}} \ln \left(\frac{1+\frac{r_{12}}{r_{1}}}{1-\frac{r_{12}}{r_{1}}}\right)=\int_{0}^{r_{12}} \frac{d r_{1}}{r_{1}} \ln \left(\frac{1+\frac{r_{1}}{r_{12}}}{1-\frac{r_{1}}{r_{12}}}\right)=\frac{\pi^{2}}{4}
\end{aligned}
$$

It can be shown that the integrals diverging at $\mathbf{y}_{1}$ involve in general a logarithm $\ln \left(r_{12} / s_{1}\right)$ but never any $\pi^{2}$ terms. The procedure we have just described indeed permits calculating most of the elementary integrals. Consider for instance the integral

$$
{ }_{i}^{\mathcal{G}} \underset{(j, 0)}{\mathcal{G}}=\int \frac{d^{3} \mathbf{x}^{\prime}}{-4 \pi} \frac{1}{\left|\mathbf{x}-\mathbf{x}^{\prime}\right|}{ }_{i} g \partial_{j}^{\prime} \operatorname{Pf} \frac{1}{r_{1}^{\prime}}
$$

(in which we replaced $g_{\text {match }}$ by $g=\ln S$ since they merely differ by a constant). We are interested in the value of this integral at point 1, following the regularization. From (5.17b) we know that the distributional part of the derivative will not contribute. Then, using (5.18a), we readily find

$$
\left({ }_{i} \mathcal{G}_{1(j, 0)}\right)_{1}=\operatorname{Pf} \int \frac{d^{3} \mathbf{x}}{-4 \pi} \frac{1}{r_{1}}{ }_{i} g \partial_{j} \frac{1}{r_{1}}+\frac{\delta^{i j}}{6 r_{12}}\left[\ln \left(\frac{r_{1}^{\prime}}{s_{1}}\right)-1\right] .
$$

The non-compact integral has a sole divergence at point 1, so that we can apply the previous method without any change, and get

$$
\operatorname{Pf} \int \frac{d^{3} \mathbf{x}}{-4 \pi} \frac{1}{r_{1}}{ }_{i} g \partial_{j} \frac{1}{r_{1}}=-\frac{\delta^{i j}}{18 r_{12}}-\frac{n_{12}^{i} n_{12}^{j}}{12 r_{12}}-\frac{\delta^{i j}}{6 r_{12}} \ln \left(\frac{r_{12}}{s_{1}}\right) .
$$

As expected the constant $\ln s_{1}$ cancels out and we arrive at:

$$
\left({ }_{i} \mathcal{G}_{(j, 0)}\right)_{1}=-\frac{2 \delta^{i j}}{9 r_{12}}-\frac{n_{12}^{i} n_{12}^{j}}{12 r_{12}}-\frac{\delta^{i j}}{6 r_{12}} \ln \left(\frac{r_{12}}{r_{1}^{\prime}}\right) .
$$

A few elementary integrals diverge at the locations $\mathbf{y}_{1}$ and $\mathbf{y}_{2}$ of both particles. In this occurrence, the integral $\int_{\mathbb{R}^{3} \backslash \mathcal{B}_{1}(s)} d^{3} \mathbf{x} F$ has no meaning, and the previous integration process 
is not adequate anymore. However, Proposition 2 in Section IV B of 47] allows us to extend it to this case. We introduce the auxiliary source

$$
\widetilde{F}_{2}=F-\sum_{b+3 \leq 0} r_{2}^{b} f_{b}
$$

which is locally integrable near $\mathbf{y}_{2}$ but does not converge at infinity. As before, the angular integral of $\tilde{F}_{2}$ around $\mathbf{y}_{1}$ takes a different expression depending on whether $r_{1} \leq r_{12}$ or $r_{1}>r_{12}$, so we must split the radial integration into the two domains $] 0, r_{12}[$ and $] r_{12},+\infty[$. Then, with full generality, the partie-finie integral of the source $F$ is given by

$$
\begin{aligned}
\operatorname{Pf}_{s_{1}, s_{2}} \int d^{3} \mathbf{x} F & =\operatorname{Pf}_{s_{1}} \int_{0}^{r_{12}} d r_{1} r_{1}^{2} \int d \Omega_{1} \widetilde{F}_{2}+\int_{r_{12}}^{+\infty} d r_{1} r_{1}^{2} \int d \Omega_{1}\left[\widetilde{F}_{2}+\frac{1}{r_{1}^{3}}\left(r_{2}^{3} F\right)_{2}\right] \\
& +4 \pi\left(r_{2}^{3} F\right)_{2} \ln \left(\frac{r_{12}}{s_{2}}\right) .
\end{aligned}
$$

If this integral comes from a Poisson integral evaluated at 1 , the constant $s_{1}$ will be cancelled out as we have seen previously and replaced by $r_{1}^{\prime}$; but there will remain in general a constant $s_{2}$ coming from the singularity at the other point. With the angular method we were able to obtain all the elementary integrals (and their gradients) at point 1 . See an appendix of [52 for the complete list of those results.

b. Analytical continuation method. The equivalence between the Hadamard partie finie prescription for integrals and the analytic continuation regularization has long been known (see e.g. [45]), and we have recovered it in the three-dimensional case by the Theorem 2 of [47. More precisely, for any $F \in \mathcal{F}$ that behaves like $o\left(1 / r^{3}\right)$ when $r \rightarrow+\infty$, the integral $\int d^{3} \mathbf{x}\left(r_{1} / s_{1}\right)^{\alpha}\left(r_{2} / s_{2}\right)^{\beta} F$ of two complex variables $\alpha$ and $\beta$ admits an analytic continuation in the neighbourhood of $\alpha=\beta=0$, and we have

$$
\operatorname{Pf}_{s_{1}, s_{2}} \int d^{3} \mathbf{x} F=\mathrm{FP}_{\substack{\alpha \rightarrow 0 \\ \beta \rightarrow 0}} \int d^{3} \mathbf{x}\left(\frac{r_{1}}{s_{1}}\right)^{\alpha}\left(\frac{r_{2}}{s_{2}}\right)^{\beta} F=\operatorname{FP}_{\substack{\beta \rightarrow 0 \\ \alpha \rightarrow 0}} \int d^{3} \mathbf{x}\left(\frac{r_{1}}{s_{1}}\right)^{\alpha}\left(\frac{r_{2}}{s_{2}}\right)^{\beta} F,
$$

where $\mathrm{FP}_{\substack{\alpha \rightarrow 0 \\ \beta \rightarrow 0}}$ means taking the finite part of the Laurent expansion of the (analytic continuation of the) integral when $\alpha \rightarrow 0$ and $\beta \rightarrow 0$ successively. This result is particularly useful in the case where $F$ is of the type $r_{1}^{p} r_{2}^{q}$, with $p$ and $q$ relative integers, since the integral is directly computable thanks to the Riesz formula [51]:

$$
\int d^{3} \mathbf{x} r_{1}^{\alpha+p} r_{2}^{\beta+q}=\pi^{\frac{3}{2}} \frac{\Gamma\left(\frac{\alpha+p+3}{2}\right) \Gamma\left(\frac{\beta+q+3}{2}\right) \Gamma\left(-\frac{\alpha+\beta+p+q+3}{2}\right)}{\Gamma\left(-\frac{\alpha+p}{2}\right) \Gamma\left(-\frac{\beta+q}{2}\right) \Gamma\left(\frac{\alpha+\beta+p+q+6}{2}\right)} r_{12}^{\alpha+\beta+p+q+3} .
$$

One may consult [47 for an example of practical computation. Most of the time, the structure of the sources of elementary integrals is more complicated than a simple $r_{1}^{p} r_{2}^{q}$, notably it involves many "free" tensorial indices which imply that generally the sources of elementary integrals, when fully developped, involve numerous inverse powers of $S=$ 
$r_{1}+r_{2}+r_{12}$. However, by considering all the possible contractions of these free indices with vectors $n_{12}^{i}$ and Kronecker symbols $\delta^{i j}$, it happens that we reduce the computation to that of several scalar integrals we can obtain thanks to the Riesz formula (5.27) (i.e., when performing the contractions and after simplification of the result, we are always led to the simple structure $r_{1}^{p} r_{2}^{q}$ without $1 / S$ powers). It should be noted that the set of scalar functions that we compute contains the complete information about the complicated tensorial integral, i.e. it permits to reconstitute it exactly.

Let us illustrate the method with the computation of the integral (5.23). It involves two free indices $i j$ and is necessarily of the type

$$
\operatorname{Pf} \int \frac{d^{3} \mathbf{x}}{-4 \pi} \frac{1}{r_{1}}{ }_{i} g \partial_{j} \frac{1}{r_{1}}=\phi\left(r_{12}\right) n_{12}^{i} n_{12}^{j}+\psi\left(r_{12}\right) \delta^{i j},
$$

where $\phi$ and $\psi$ are some unknown "scalars" depending on $r_{12}$. By contracting successively the integrand with $n_{12}^{i} n_{12}^{j}$ and $\delta^{i j}$, and simplifying, we find:

$$
\begin{aligned}
n_{12}^{i} n_{12}^{j}{ }_{i} g \partial_{j} \frac{1}{r_{1}} & =\frac{1}{4 r_{1}^{3}}-\frac{r_{2}}{4 r_{1}^{4}}+\frac{1}{4 r_{1} r_{12}^{2}}-\frac{r_{2}}{4 r_{1}^{2} r_{12}^{2}}-\frac{r_{2}^{2}}{4 r_{1}^{3} r_{12}^{2}} \\
& +\frac{r_{2}^{3}}{4 r_{1}^{4} r_{12}^{2}}+\frac{1}{4 r_{1}^{2} r_{12}}-\frac{r_{2}^{2}}{4 r_{1}^{4} r_{12}}+\frac{r_{12}}{4 r_{1}^{4}}, \\
\delta^{i j}{ }_{i} g \partial_{j} \frac{1}{r_{1}} & =\frac{1}{2 r_{1}^{3}}+\frac{1}{2 r_{1}^{2} r_{12}}-\frac{r_{2}}{2 r_{1}^{3} r_{12}},
\end{aligned}
$$

thus obtaining a sum of terms of the type $r_{1}^{p} r_{2}^{q}$ which can all be integrated with the help of the formula (5.27). This computation yields a system of equations for the scalars $\phi$ and $\psi$ :

$$
\begin{aligned}
& \phi+\psi=-\frac{5}{36 r_{12}}-\frac{1}{6 r_{12}} \ln \left(\frac{r_{12}}{s_{1}}\right), \\
& \phi+3 \psi=-\frac{1}{4 r_{12}}-\frac{1}{2 r_{12}} \ln \left(\frac{r_{12}}{s_{1}}\right) .
\end{aligned}
$$

By solving the previous system, and inserting the results into (5.28), we recover exactly the value given by (5.23). We have checked in this manner all the elementary integrals previously computed with the angular method.

\section{Finite part of integrals diverging at infinity}

For the moment, we have left aside the case where our integrals are defined by means of a finite part dealing with a divergence occuring at infinity. Such a study is needed to compute some 0.5PN integrals encountered in Section [V. From now on, we suppose that the source $F$ admits an expansion when $r \rightarrow+\infty$ which is made of simple powers $r^{n-3}$, with

$n \leq n_{\max }$ (so the integral a priori diverges at infinity when $n_{\max } \geq 0$ ), and we consider the quantity: 


$$
\operatorname{Pf}\left\{\mathrm{FP}_{B \rightarrow 0} \int d^{3} \mathbf{x}\left(\frac{r}{r_{0}}\right)^{B} F\right\} .
$$

We split the integral above into two integrals $I_{\text {int }}$ and $I_{\text {ext }}$ extending respectively over a domain $D_{\text {int }}$ including the two local singularities $\mathbf{y}_{1,2}$, and the complementary domain $D_{\text {ext }}$ comprising the regions at infinity. Using the integration variable $\mathbf{r}_{1}=\mathbf{x}-\mathbf{y}_{1}$, the external integral (on which the regularization Pf can be removed) reads as

$$
I_{\text {ext }}=\mathrm{FP}_{B \rightarrow 0} \int_{D_{\mathrm{ext}}} d^{3} \mathbf{r}_{\mathbf{1}}\left(\frac{\left|\mathbf{r}_{1}+\mathbf{y}_{1}\right|}{r_{0}}\right)^{B} F\left(\mathbf{r}_{\mathbf{1}}+\mathbf{y}_{1}\right) .
$$

If we assume that the original integral can generate only simple poles $\sim 1 / B$ at infinity (which will be always the case here), we can replace it by

$$
\begin{aligned}
I_{\text {ext }} & =\mathrm{FP}_{B \rightarrow 0} \int_{D_{\text {ext }}} d^{3} \mathbf{r}_{1}\left(\frac{r_{1}}{r_{0}}\right)^{B} F \\
& +\mathrm{FP}_{B \rightarrow 0} \frac{B}{2} \int_{D_{\text {ext }}} d^{3} \mathbf{r}_{1}\left(\frac{r_{1}}{r_{0}}\right)^{B} \ln \left(1+2\left(n_{1} y_{1}\right) \frac{1}{r_{1}}+\frac{y_{1}^{2}}{r_{1}^{2}}\right) F
\end{aligned}
$$

Indeed, in the case of simple poles, the other terms, involving at least a factor $B^{2}$, will always give zero. The second term is non-zero only if the corresponding integral does admit a pole, and can be calculated in a simple way by picking up the term of order $1 / r_{1}^{3}$ in the expansion of the integrand when $r \rightarrow+\infty$. Notably, in the important case where the function $F$ goes to zero like $1 / r^{3}$ at infinity, its product with the log-term behaves at least like $1 / r^{4}$ and therefore the second term in (5.30) gives no contribution. For instance, an integral divergent at infinity that we encounter in the problem is

$$
\mathrm{FP}_{B \rightarrow 0} \int d^{3} \mathbf{x}\left(\frac{r}{r_{0}}\right)^{B} \partial_{i j}\left(\mathrm{Pf} \frac{1}{r_{1}}\right)=-\frac{4 \pi}{3} \delta_{i j} .
$$

\section{E. Lorentzian regularization of potentials}

All the potentials and their gradients (compact-support, quadratic and non-compact potentials) which have been computed in this section and the previous one were obtained at the point 1 using the standard Hadamard regularization $(F)_{1}$. However, this regularization, being defined within the hypersurface $t=$ const of the harmonic coordinates, must break at some point the Lorentz-invariance properties of the potentials. That is, if a potential defined for a smooth "fluid" behaves in a certain way under a Lorentz transformation, we expect that its regularized value at the point 1 in the sense of $(F)_{1}$ will generically not behave in the same way. Nevertheless, the equations of motion in harmonic coordinates, as computed with the regularization $(F)_{1}$, are known to be Lorentz invariant up to the 2.5PN order 31. Perhaps not surprisingly because of this fact, it turns out that the Lorentzian 
regularization $[F]_{1}$ (defined in [48]) yields no difference with respect to the old regularization $(F)_{1}$ for all the $3 \mathrm{PN}$ potentials but for one, namely the cubic-non-compact potential $\hat{X}^{(\mathrm{CNC})}$ defined by (5.1) and which had to be computed at the relative 1PN order. (Evidently we have $[F]_{1}=(F)_{1}$ for all the potentials which are to be computed with Newtonian accuracy.) Thus all the results obtained so far but the one for $\left(\partial_{i} \hat{X}^{(\mathrm{CNC})}\right)_{1}$ [the relevant quantity for the equations of motion] are valid in the case of the new regularization. In this subsection we compute the remaining part $\left[\partial_{i} \hat{X}^{(\mathrm{CNC})}\right]_{1}-\left(\partial_{i} \hat{X}^{(\mathrm{CNC})}\right)_{1}$.

Since the regularization $[F]_{1}$ brings some new terms with respect to the old one $(F)_{1}$ starting at the relative $1 \mathrm{PN}$ order, and since $\hat{X}^{(\mathrm{CNC})}$ is to be computed at the $1 \mathrm{PN}$ order only, it is sufficient for this calculation to use the lowest-order, Newtonian value of $\hat{X}^{(\mathrm{CNC})}$. From the computation in [57,31] we know the analytic closed-form expression of $\hat{X}^{(\mathrm{CNC})}$ at Newtonian order for any field point $(t, \mathbf{x})$,

$$
\hat{X}^{(\mathrm{CNC})}=\frac{G^{3} m_{1}^{3}}{12 r_{1}^{3}}-G^{3} m_{1}^{2} m_{2}\left\{\frac{1}{8 r_{2} r_{12}^{2}}+\frac{1}{16} K_{1}+H_{1}\right\}+\mathcal{O}(1)+1 \leftrightarrow 2,
$$

where the functions $K_{1}$ and $H_{1}$, which are solutions of certain Poisson equations, are given explicitly by (5.15). The complete developed forms of these functions are

$$
\begin{aligned}
& K_{1}=-\frac{1}{r_{2}^{3}}+\frac{1}{r_{2} r_{12}^{2}}-\frac{1}{r_{1}^{2} r_{2}}+\frac{r_{2}}{2 r_{1}^{2} r_{12}^{2}}+\frac{r_{12}^{2}}{2 r_{1}^{2} r_{2}^{3}}+\frac{r_{1}^{2}}{2 r_{2}^{3} r_{12}^{2}}, \\
& H_{1}=-\frac{1}{2 r_{1}^{3}}-\frac{1}{4 r_{12}^{3}}-\frac{1}{4 r_{1}^{2} r_{12}}-\frac{r_{2}}{2 r_{1}^{2} r_{12}^{2}}+\frac{r_{2}}{2 r_{1}^{3} r_{12}}+\frac{3 r_{2}^{2}}{4 r_{1}^{2} r_{12}^{3}}+\frac{r_{2}^{2}}{2 r_{1}^{3} r_{12}^{2}}-\frac{r_{2}^{3}}{2 r_{1}^{3} r_{12}^{3}} .
\end{aligned}
$$

We replace these expressions into (5.31), and we implement all the rules for the new regularization $[F]_{1}$ defined in Section III of [48]. Equivalently, since the order of the computation is limited to $1 \mathrm{PN}$, we can use the closed form formula

$$
[F]_{1}-(F)_{1}=\frac{1}{c^{2}}\left(\left(\mathbf{r}_{1} \cdot \mathbf{v}_{1}\right)\left[\partial_{t} F+\frac{1}{2} v_{1}^{i} \partial_{i} F\right]\right)_{1}+\mathcal{O}(4),
$$

derived in Section IV of [48]. As a result, we obtain, for the potential itself,

$$
\begin{aligned}
{\left[\hat{X}^{(\mathrm{CNC})}\right]_{1} } & -\left(\hat{X}^{(\mathrm{CNC})}\right)_{1} \\
& =\frac{G^{3} m_{1}^{2} m_{2}}{c^{2} r_{12}^{3}}\left\{\frac{43}{40}\left(n_{12} v_{1}\right)^{2}-\left(n_{12} v_{1}\right)\left(n_{12} v_{2}\right)-\frac{43}{120} v_{1}^{2}+\frac{1}{3}\left(v_{1} v_{2}\right)\right\}+\mathcal{O}(4) .
\end{aligned}
$$

In the case of the gradient needed for the equations of motion, we get

$$
\begin{aligned}
{\left[\partial_{i} \hat{X}^{(\mathrm{CNC})}\right]_{1} } & -\left(\partial_{i} \hat{X}^{(\mathrm{CNC})}\right)_{1} \\
& =\frac{G^{3} m_{1}^{2} m_{2}}{c^{2} r_{12}^{4}}\left\{\left[\frac{27}{56}\left(n_{12} v_{1}\right)^{2}-\frac{3}{4}\left(n_{12} v_{1}\right)\left(n_{12} v_{2}\right)-\frac{27}{280} v_{1}^{2}+\frac{3}{20}\left(v_{1} v_{2}\right)\right] n_{12}^{i}\right. \\
& \left.-\frac{27}{140}\left(n_{12} v_{1}\right) v_{1}^{i}+\frac{3}{20}\left(n_{12} v_{2}\right) v_{1}^{i}+\frac{3}{20}\left(n_{12} v_{1}\right) v_{2}^{i}\right\}+\mathcal{O}(4) .
\end{aligned}
$$


As we see, the new regularization brings some definite non-zero contributions at the $1 \mathrm{PN}$ order in the case of this potential, which will constitute a crucial contribution to the $3 \mathrm{PN}$ equations of motion. The right-hand side of (5.35) is not invariant by itself under a Lorentz transformation - it cannot be - , but will ensure finally the Lorentz-invariance of the 3PN equations of motion.

\section{LEIBNIZ TERMS AND NON-DISTRIBUTIVITY}

\section{A. Effect of a gauge transformation}

In this subsection we study the effect of a gauge transformation on the $3 \mathrm{PN}$ equations of motion as well as energy of the two particles. Let $\left\{x^{\mu}\right\}$ denote the harmonic coordinate system and $g_{\mu \nu}(x)$ be the harmonic-coordinate metric, generated by the two particles, that we have iterated in previous sections up to the $3 \mathrm{PN}$ order. The metric depends on the position $\mathbf{x}$ of the field point, and on the coordinate time $t=x^{0} / c$ through the trajectories $\mathbf{y}_{1,2}(t)$ and velocities $\mathbf{v}_{1,2}(t)$ of the particles, i.e.

$$
g_{\mu \nu}(x)=g_{\mu \nu}\left[\mathbf{x} ; \mathbf{y}_{1}(t), \mathbf{y}_{2}(t) ; \mathbf{v}_{1}(t), \mathbf{v}_{2}(t)\right]
$$

We know that the dependence of the metric over the velocities arises at the 1PN order (see e.g. the equations (7.2) in [31]), namely the order $\mathcal{O}(4,3,4)$, where this notation is a shorthand for saying $\mathcal{O}(4)=\mathcal{O}\left(1 / c^{4}\right)$ in $g_{00}, \mathcal{O}(3)$ in $g_{0 i}$ and $\mathcal{O}(4)$ in $g_{i j}$. Consider an infinitesimal coordinate transformation of the type

$$
\begin{aligned}
& x^{\prime \mu}=x^{\mu}+\xi^{\mu}(x), \\
& \xi^{\mu}(x)=\xi^{\mu}\left[\mathbf{x} ; \mathbf{y}_{1}(t), \mathbf{y}_{2}(t)\right],
\end{aligned}
$$

where, in order to simplify the presentation, we assume that the gauge vector $\xi^{\mu}$ depends on the positions $\mathbf{y}_{1,2}$ of the particles, but not on their velocities. Furthermore, we suppose that this gauge transformation is at the level of the $3 \mathrm{PN}$ order, which means that $\xi^{0}=\mathcal{O}(7)$ and $\xi^{i}=\mathcal{O}(6)$, or equivalently $\xi^{\mu}=\mathcal{O}(7,6)$. In addition, in a first stage, we suppose that the vector $\xi^{\mu}(x)$ is a smooth function of the coordinates even at the positions of the particles. The new metric in the new coordinate system $\left\{x^{\prime \mu}\right\}$ is

$$
g_{\mu \nu}^{\prime}\left(x^{\prime}\right)=g_{\mu \nu}^{\prime}\left[\mathbf{x}^{\prime} ; \mathbf{y}_{1}^{\prime}\left(t^{\prime}\right), \mathbf{y}_{2}^{\prime}\left(t^{\prime}\right) ; \mathbf{v}_{1}^{\prime}\left(t^{\prime}\right), \mathbf{v}_{2}^{\prime}\left(t^{\prime}\right)\right]
$$

where the new trajectories and velocities $\mathbf{y}_{1,2}^{\prime}, \mathbf{v}_{1,2}^{\prime}$ are parametrized by the new coordinate time $t^{\prime}=x^{\prime 0} / c$. The coordinate change (6.2), when applied at the location of each of the

particles, yields the relations between the new and old trajectories, which, when retaining only the terms up to the order $\mathcal{O}(6)$, read as 


$$
\begin{aligned}
& y_{1}^{\prime i}\left(t^{\prime}\right)=y_{1}^{i}(t)+\xi^{i}\left(y_{1}\right)+\mathcal{O}(8) \\
& y_{2}^{\prime i}\left(t^{\prime}\right)=y_{2}^{i}(t)+\xi^{i}\left(y_{2}\right)+\mathcal{O}(8)
\end{aligned}
$$

where the $\xi^{\mu}\left(y_{1,2}\right)$ 's denote the gauge vector at the position of the particles, for instance $\xi^{\mu}\left(y_{1}\right)=\xi^{\mu}\left[\mathbf{y}_{1}(t) ; \mathbf{y}_{1}(t), \mathbf{y}_{2}(t)\right]$. The new metric (6.3), when expressed in terms of the old variables, follows from this as

$$
g_{\mu \nu}^{\prime}\left(x^{\prime}\right)=g_{\mu \nu}^{\prime}(x)+\xi^{i}(x) \partial_{i} g_{\mu \nu}+\xi^{i}\left(y_{1}\right)_{1} \partial_{i} g_{\mu \nu}+\xi^{i}\left(y_{2}\right)_{2} \partial_{i} g_{\mu \nu}+\mathcal{O}(10,9,10)
$$

where we have used the fact that the dependence of the metric on the velocities starts at the $1 \mathrm{PN}$ order, so the terms due to the modification of the velocities do not contribute to (6.5). Since the $3 \mathrm{PN}$ metric depends on space $\mathbf{x}$ only through the two distances $\mathbf{x}-\mathbf{y}_{1}$ and $\mathbf{x}-\mathbf{y}_{2}$, we have $\partial_{i} g_{\mu \nu}+{ }_{1} \partial_{i} g_{\mu \nu}+{ }_{2} \partial_{i} g_{\mu \nu}=0$, and so an equivalent form of (6.5) is

$$
g_{\mu \nu}^{\prime}\left(x^{\prime}\right)=g_{\mu \nu}^{\prime}(x)+\left[\xi^{i}\left(y_{1}\right)-\xi^{i}(x)\right]_{1} \partial_{i} g_{\mu \nu}+\left[\xi^{i}\left(y_{2}\right)-\xi^{i}(x)\right]_{2} \partial_{i} g_{\mu \nu}+\mathcal{O}(10,9,10) .
$$

The equation (6.6), when combined with the law of transformation of tensors, i.e. in the present case

$$
g_{\mu \nu}(x)=g_{\mu \nu}^{\prime}\left(x^{\prime}\right)+\partial_{\mu} \xi_{\nu}+\partial_{\nu} \xi_{\mu}+\mathcal{O}(10,9,8)
$$

where $\xi_{\mu}=\eta_{\mu \nu} \xi^{\nu}$, gives the metric variation or Lie derivative $\delta_{\xi} g_{\mu \nu}=g_{\mu \nu}^{\prime}(x)-g_{\mu \nu}(x)$ (where the same variable $x$ is used for both the transformed and original metrics) as

$$
\delta_{\xi} g_{\mu \nu}=-\partial_{\mu} \xi_{\nu}-\partial_{\nu} \xi_{\mu}+\left[\xi^{i}(x)-\xi^{i}\left(y_{1}\right)\right]_{1} \partial_{i} g_{\mu \nu}+\left[\xi^{i}(x)-\xi^{i}\left(y_{2}\right)\right]_{2} \partial_{i} g_{\mu \nu}+\mathcal{O}(10,9,8)
$$

In fact, up to this order, only the 00 component of the metric includes a "non-linear" correction term; and, within that non-linear term, the metric can be approximated by its Newtonian part, so

$$
\begin{aligned}
\delta_{\xi} g_{00} & =-2 \partial_{0} \xi_{0}+\frac{2}{c^{2}}\left(\left[\xi^{i}(x)-\xi^{i}\left(y_{1}\right)\right]_{1} \partial_{i} U+\left[\xi^{i}(x)-\xi^{i}\left(y_{2}\right)\right]_{2} \partial_{i} U\right)+\mathcal{O}(10) \\
\delta_{\xi} g_{0 i} & =-\partial_{0} \xi_{i}-\partial_{i} \xi_{0}+\mathcal{O}(9) \\
\delta_{\xi} g_{i j} & =-\partial_{i} \xi_{j}-\partial_{j} \xi_{i}+\mathcal{O}(8)
\end{aligned}
$$

where $U=\frac{G m_{1}}{r_{1}}+\frac{G m_{2}}{r_{2}}$ is the Newtonian potential (with a small inconsistency of notation with respect to previous sections). Now, it is easy to check that, in the sense of distributions,

$$
\Delta\left(\left[\xi^{i}(x)-\xi^{i}\left(y_{1}\right)\right]_{1} \partial_{i} U+\left[\xi^{i}(x)-\xi^{i}\left(y_{2}\right)\right]_{2} \partial_{i} U\right)=-2 \partial_{i} \xi_{j} \partial_{i j} U-\Delta \xi_{i} \partial_{i} U
$$


Indeed, the delta-functions at the points 1 and 2, which come from the Laplacian of $U$, are killed respectively by the factors $\xi^{i}(x)-\xi^{i}\left(y_{1}\right)$ and $\xi^{i}(x)-\xi^{i}\left(y_{2}\right)$, which vanish respectively at these two points, in front of them. So, we can write for $\delta_{\xi} g_{00}$ the simpler but equivalent expression

$$
\delta_{\xi} g_{00}=-2 \partial_{0} \xi_{0}-\frac{2}{c^{2}} \Delta^{-1}\left[2 \partial_{i} \xi_{j} \partial_{i j} U+\Delta \xi_{i} \partial_{i} U\right]+\mathcal{O}(10)
$$

where $\Delta^{-1}$ denotes the usual Poisson integral.

The latter result can be generalized to our framework of singular metrics by allowing the gauge vector $\xi^{\mu}$ to become singular at the positions of the particles (in the sense that $\left.\xi^{\mu} \in \mathcal{F}\right)$, provided that the integral appearing in $(\underline{6.10})$ is treated as the Hadamard partie finie of a Poisson integral in the way which is investigated in Section $\mathrm{V}$ of [47]. Let us consider, for example, the $3 \mathrm{PN}$ gauge vector given by

$$
\xi_{\mu}=\frac{G^{3} m^{3}}{c^{6}} \partial_{\mu}\left(\frac{\epsilon_{1}}{r_{1}}+\frac{\epsilon_{2}}{r_{2}}\right)
$$

where $\epsilon_{1}$ and $\epsilon_{2}$ denote two dimensionless constants or possibly functions of time $t$ (and where $\left.m=m_{1}+m_{2}\right)$. Note that with this choice of gauge vector the new coordinates satisfy the condition of harmonic coordinates outside the singularities (i.e. in the sense of functions) at the 3PN order: indeed $\square \xi^{\mu}=\mathcal{O}(9,8)$. When inserting (6.11) into (6.10), we must be careful about evaluating the last term of (6.10) in the sense of distributions, taking into account the fact that $\Delta \xi_{i}$ is distributional. For this term we obtain

$$
\Delta^{-1}\left[\Delta \xi_{i} \partial_{i} U\right]=\frac{G^{3} m^{3}}{c^{6}}\left[\gamma_{1}^{i} \partial_{i}\left(\frac{\epsilon_{1}}{r_{1}}\right)+\gamma_{2}^{i} \partial_{i}\left(\frac{\epsilon_{2}}{r_{2}}\right)\right]
$$

where $\gamma_{1}^{i}$ and $\gamma_{2}^{i}$ are the Newtonian accelerations of 1 and 2. Therefore, we find

$$
\delta_{\xi} g_{00}=-\frac{2 G^{3} m^{3}}{c^{8}}\left\{\left[\partial_{t}^{2}+\gamma_{1}^{i} \partial_{i}\right]\left(\frac{\epsilon_{1}}{r_{1}}\right)+2 \Delta^{-1}\left[\partial_{i j}\left(\frac{\epsilon_{1}}{r_{1}}\right) \partial_{i j} U\right]\right\}+\mathcal{O}(10)+1 \leftrightarrow 2 .
$$

In the case where $\epsilon_{1}$ and $\epsilon_{2}$ are some pure constants (independent on time) we can somewhat simplify the latter expression by using the fact that the accelerations cancel out in the first term. In this case, we obtain the full metric transformations as

$$
\begin{aligned}
& \delta_{\xi} g_{00}=-\frac{2 G^{3} m^{3}}{c^{8}}\left\{v_{1}^{i j} \partial_{i j}\left(\frac{\epsilon_{1}}{r_{1}}\right)+2 \Delta^{-1}\left[\partial_{i j}\left(\frac{\epsilon_{1}}{r_{1}}\right) \partial_{i j} U\right]\right\}+\mathcal{O}(10)+1 \leftrightarrow 2, \\
& \delta_{\xi} g_{0 i}=\frac{2 G^{3} m^{3}}{c^{7}} v_{1}^{j} \partial_{i j}\left(\frac{\epsilon_{1}}{r_{1}}\right)+\mathcal{O}(9)+1 \leftrightarrow 2, \\
& \delta_{\xi} g_{i j}=-\frac{2 G^{3} m^{3}}{c^{6}} \partial_{i j}\left(\frac{\epsilon_{1}}{r_{1}}\right)+\mathcal{O}(8)+1 \leftrightarrow 2 .
\end{aligned}
$$


By comparing this with the 3PN metric (3.24), we see that the gauge transformation induces the following changes in the $3 \mathrm{PN}$ potentials $\hat{T}, \hat{Y}_{i}$ and $\hat{Z}_{i j}$ :

$$
\begin{aligned}
\delta_{\xi} \hat{T} & =-\frac{G^{3} m^{3}}{16}\left\{v_{1}^{i j} \partial_{i j}\left(\frac{\epsilon_{1}}{r_{1}}\right)+2 \Delta^{-1}\left[\partial_{i j}\left(\frac{\epsilon_{1}}{r_{1}}\right) \partial_{i j} U\right]\right\}+1 \leftrightarrow 2, \\
\delta_{\xi} \hat{Y}_{i} & =-\frac{G^{3} m^{3}}{8} v_{1}^{j} \partial_{i j}\left(\frac{\epsilon_{1}}{r_{1}}\right)+1 \leftrightarrow 2 \\
\delta_{\xi} \hat{Z}_{i j} & =-\frac{G^{3} m^{3}}{8} \partial_{i j}\left(\frac{\epsilon_{1}}{r_{1}}\right)+1 \leftrightarrow 2 .
\end{aligned}
$$

The computation of the non-linearity term in (6.14a) is straightforward, and we get

$$
\Delta^{-1}\left[\partial_{i j}\left(\frac{1}{r_{1}}\right) \partial_{i j} U\right]=\frac{G m_{1}}{2 r_{1}^{4}}+G m_{2 i j} g_{i j},
$$

where $g=\ln \left(r_{1}+r_{2}+r_{12}\right)$ is a kernel satisfying $\Delta g=\frac{1}{r_{1} r_{2}}$ [see also (4.36)], and where we denote $_{i j} g_{i j}=\partial_{1 i j} \partial_{2 i j} g=D^{2} g$. At last, we insert the latter changes of the 3PN potentials into the (regularized) equations of motion (3.32) with (3.35) (there is no need to include a correction due to the non-distributivity), and obtain the corresponding change in the acceleration of the particle 1 as

$$
\begin{aligned}
\delta_{\xi} a_{1}^{i} & =\frac{2 G^{4} m^{3}}{c^{6} r_{12}^{5}}\left(\epsilon_{1} m_{2}-\epsilon_{2} m_{1}\right) n_{12}^{i} \\
& +\frac{G^{3} m^{3} \epsilon_{2}}{c^{6} r_{12}^{4}}\left[-15\left(n_{12} v_{12}\right)^{2} n_{12}^{i}+3 v_{12}^{2} n_{12}^{i}+6\left(n_{12} v_{12}\right) v_{12}^{i}\right] .
\end{aligned}
$$

In the case where $\epsilon_{1}$ and $\epsilon_{2}$ depend on time, there are some extra contributions proportional to $\dot{\epsilon}_{2}$ and $\ddot{\epsilon}_{2}$. A good check of (6.15) is the fact that to the change in the acceleration (6.15) always corresponds a change in the associated energy; that is, the gauge transformation does not modify the existence of a conserved energy (see Section VII for the computation of the 3PN energy). Namely, we find that the combination $m_{1} \delta_{\xi} a_{1}^{i} v_{1}^{i}+m_{2} \delta_{\xi} a_{2}^{i} v_{2}^{i}$ is a total time-derivative, and from this we obtain the gauge transformation of the energy as

$$
\delta_{\xi} E=\epsilon_{2} \frac{G^{3} m^{3} m_{1}}{c^{6} r_{12}^{3}}\left[\frac{G m_{2}}{r_{12}}-3\left(n_{12} v_{1}\right)\left(n_{12} v_{12}\right)+\left(v_{1} v_{12}\right)\right]+1 \leftrightarrow 2
$$

\section{B. Leibniz contributions}

An important ingredient of the present computation is the novel distributional derivative associated with the Hadamard regularization which has been introduced in 477 (see also Section [1]). This derivative permits us to derive in a systematic and consistent way all the integrals encountered in the problem; however it represents merely a mathematical tool, which is maybe not connected to any relevant Physics. Therefore, it is important to know 
exactly the role played by this derivative in the 3PN equations of motion, with respect to say the Schwartz distributional derivative 45. We know that our distributional derivative affects the computation of two types of terms: (i) the "self" terms entering a priori in the non-linear potentials $\hat{X}, \hat{T}$ and $\hat{Y}_{i}$ and which are ill-defined in the case of the Schwartz derivative (see Section $\nabla$ ), (ii) the "Leibniz" terms which account for the violation of the Leibniz rule during the $3 \mathrm{PN}$ iteration of the metric as discussed in Section [II. In the present subsection, we compute the Leibniz terms and combine the result with the one of Section $\mathrm{V}$ concerning the self terms. The conclusion is that the terms coming from the use of the distributional derivative are necessary for keeping track of the Lorentz invariance of the equations of motion, and that no other Physics is involved with them in the present formalism (see also Section VII). We do the computation for both the "particular" derivative defined by (2.9) and the more correct one given by (2.10)-(2.11).

The Leibniz terms discussed in Section [II] consist of those contributions of the type (3.23) and alike which arise in the process of simplification of the $3 \mathrm{PN}$ metric $h^{\mu \nu}$ by means of the Leibniz rule. These terms depend only on the distributional part of the derivative, $\mathrm{D}_{i}^{\text {part }}[F]$ or $\mathrm{D}_{i}[F]$. The formulas giving the complete Leibniz terms in $h^{\mu \nu}$, not being very attractive, are relegated to Appendix A. When reducing explicitly these formulas we find that all the terms take the same simple structure and, not surprisingly, arise only at the 3PN order. As already announced in (3.27), the Leibniz terms imply a priori a net contribution to the $3 \mathrm{PN}$ potentials $\hat{T}, \hat{Y}_{i}$ and $\hat{Z}_{i j}$. Actually, in the case of the particular derivative, their contributions to the vector and tensor potentials $\hat{Y}_{i}$ and $\hat{Z}_{i j}$ turn out to be zero,

$$
\begin{aligned}
& \delta_{\text {Leibniz }} \hat{Y}_{i}=0, \\
& \delta_{\text {Leibniz }} \hat{Z}_{i j}=0,
\end{aligned}
$$

while the contribution to the scalar potential $\hat{T}$, also in the case of $\mathrm{D}_{i}^{\text {part }}[F]$, is found to be

$$
\delta_{\text {Leibniz }} \hat{T}=-\frac{G^{3} m_{1}^{3}}{96} v_{1}^{i} v_{1}^{j} \partial_{i j}\left(\frac{1}{r_{1}}\right)+\frac{11}{36} \frac{G^{4} m_{1}^{3} m_{2}}{r_{12}^{2}} n_{12}^{i} \partial_{i}\left(\frac{1}{r_{1}}\right)+1 \leftrightarrow 2 .
$$

The modification of the acceleration of body 1 which is generated by the latter Leibniz terms reads as

$$
\delta_{\text {Leibniz }} a_{1}^{i}=\frac{G^{3} m_{2}^{3}}{c^{6} r_{12}^{4}}\left[\frac{5}{2}\left(n_{12} v_{2}\right)^{2} n_{12}^{i}-\frac{1}{2} v_{2}^{2} n_{12}^{i}-\left(n_{12} v_{2}\right) v_{2}^{i}\right]-\frac{88}{9} \frac{G^{4} m_{1} m_{2}^{3}}{c^{6} r_{12}^{5}} n_{12}^{i} .
$$

On the other hand, we computed in Sections $\mathrm{IV}$ and $\mathrm{V}$ many distributional terms associated with the derivative of the non-linear potentials in the right-hand side of the field equations [see (3.15)]. Most of these terms are simply given by the Schwartz distributional derivative. The only terms which require the new distributional derivative of [47] come from the computation of the "self" parts of the non-compact potentials (see Section $\nabla$ ). In this case, the modifications of the potentials have been found to be given by (5.7), from which we obtain the following modification of the acceleration: 


$$
\delta_{\text {self }} a_{1}^{i}=\frac{G^{3} m_{2}^{3}}{c^{6} r_{12}^{4}}\left[-\frac{1}{2}\left(n_{12} v_{2}\right)^{2} n_{12}^{i}+\frac{1}{10} v_{2}^{2} n_{12}^{i}+\frac{1}{5}\left(n_{12} v_{2}\right) v_{2}^{i}\right]+\frac{151}{9} \frac{G^{4} m_{1} m_{2}^{3}}{c^{6} r_{12}^{5}} n_{12}^{i} .
$$

Adding up (6.19) and (6.20) we therefore obtain the total effect of the (particular) distributional derivative as

$$
\delta_{\text {distribution }} a_{1}^{i}=\frac{G^{3} m_{2}^{3}}{c^{6} r_{12}^{4}}\left[2\left(n_{12} v_{2}\right)^{2} n_{12}^{i}-\frac{2}{5} v_{2}^{2} n_{12}^{i}-\frac{4}{5}\left(n_{12} v_{2}\right) v_{2}^{i}\right]+7 \frac{G^{4} m_{1} m_{2}^{3}}{c^{6} r_{12}^{5}} n_{12}^{i} .
$$

Interestingly, this quite simple piece of the acceleration of particle 1 involves the velocity of particle 2 alone, and therefore does not stay by itself invariant under a Lorentz transformations, or, rather, at this order, a Galilean transformation (indeed, for this to be true the term should depend on the relative velocity $\mathbf{v}_{12}=\mathbf{v}_{1}-\mathbf{v}_{2}$ ). Therefore, if we are correct, since we are using harmonic coordinates and have employed a Lorentzian regularization, the result (6.21) has to combine with other pieces in the acceleration so as to maintain the Lorentz invariance of the equations. We have found that this is exactly what happens: the dependence of (6.21) over the velocity $\mathbf{v}_{2}$ is mandatory for the Lorentz invariance of the final 3PN equations to work. This constitutes, in our opinion, an important check of the relevance of the distributional derivative introduced in [47]. It shows also that this derivative is merely a tool for preventing a breakdown of the Lorentz invariance when performing integrations by parts of complicated divergent integrals [the last term in (6.21), which is not checked by the Lorentz invariance, will turn out to be absorbed into the adjustement of a certain constant, see Section VII].

The previous check has been done with the "particular" distributional derivative (2.9), and it is interesting to redo the computation in the case of the distributional derivative defined by (2.10)-(2.11), that we recall is more satisfying than the particular one because it obeys the rule of commutation of successive derivatives. (But note in passing that we have verified that the particular derivative does not yield any ambiguity at the 3PN order which would be due to the non-commutation of derivatives; however, such ambiguities could arise at higher orders, in which case the "correct" derivative would be more appropriate.) In particular, while the particular derivative is entirely deterministic, the derivative (2.10)(2.11) depends on a constant $K$, and it is important to know the fate of this constant in the final equations of motion, and how the test of the Lorentz invariance will manage to be satisfied in fine. Like for the particular derivative we find that the incidence of this derivative is through two distinct contributions, Leibniz and self. Consider the Leibniz contribution: we perform exactly the same computation as before, i.e. based on the formulas in the appendix A, and find that in the case of the new derivative (2.10)-(2.11) the terms in the potentials $\hat{Y}_{i}$ and $\hat{Z}_{i j}$ are no longer zero, but are given by

$$
\left.\delta_{\text {Leibniz }} \hat{Y}_{i}\right|_{K}=\left(-\frac{1}{15}+\frac{2}{15} K\right) G^{3} m_{1}^{3} v_{1}^{j} \partial_{i j}\left(\frac{1}{r_{1}}\right)+1 \leftrightarrow 2,
$$




$$
\left.\delta_{\text {Leibniz }} \hat{Z}_{i j}\right|_{K}=\left(-\frac{1}{15}+\frac{2}{15} K\right) G^{3} m_{1}^{3} \partial_{i j}\left(\frac{1}{r_{1}}\right)+1 \leftrightarrow 2
$$

Our convention is that the explicit indication in the left-hand side of the dependence over $K$ means that the computation is performed using the "correct" distributional derivative. In the case of the modification of the potential $\hat{T}$ the things are a little more complicated because we have to take into account, in addition to a "linear" contribution similar to those of (6.22), the "non-linear" term which is generated by the modification of the tensor potential $\hat{Z}_{i j}$ shown in (6.22b); $c f$ the source term $\hat{Z}_{i j} \partial_{i j} V$ in the definition (3.27a of $\hat{T}$. We obtain

$$
\begin{aligned}
\delta_{\text {Leibniz }} \hat{T}_{K} & =\left(-\frac{53}{480}+\frac{2}{5} K\right) G^{3} m_{1}^{3} v_{1}^{i} v_{1}^{j} \partial_{i j}\left(\frac{1}{r_{1}}\right) \\
& +\left(\frac{19}{288}+\frac{47}{24} K\right) \frac{G^{4} m_{1}^{3} m_{2}}{r_{12}^{2}} n_{12}^{i} \partial_{i}\left(\frac{1}{r_{1}}\right) \\
& +\Delta^{-1}\left[\left.\delta_{\text {Leibniz }} \hat{Z}_{i j}\right|_{K} \partial_{i j} U\right]+1 \leftrightarrow 2,
\end{aligned}
$$

where $U=\frac{G m_{1}}{r_{1}}+\frac{G m_{2}}{r_{2}}$. Now, using the results of the previous subsection, we see that many of these terms are in the form of a gauge transformation corresponding to a gauge vector $\xi^{\mu}$ of the type 6.11). Indeed, we pose

$$
\begin{aligned}
& \left.\epsilon_{1}\right|_{K}=\frac{8}{15}(1-2 K)\left(\frac{m_{1}}{m}\right)^{3}, \\
& \epsilon_{2}=\frac{8}{15}(1-2 K)\left(\frac{m_{2}}{m}\right)^{3} .
\end{aligned}
$$

With this choice the Leibniz corrections in $\hat{Y}_{i}$ and $\hat{Z}_{i j}$ become pure gauge,

$$
\begin{gathered}
\left.\delta_{\text {Leibniz }} \hat{Y}_{i}\right|_{K}=\left.\delta_{\xi} \hat{Y}_{i}\right|_{K}, \\
\left.\delta_{\text {Leibniz }} \hat{Z}_{i j}\right|_{K}=\left.\delta_{\xi} \hat{Z}_{i j}\right|_{K},
\end{gathered}
$$

while we can re-write 6.23$)$ in the simplified form

$$
\begin{aligned}
\delta_{\text {Leibniz }} \hat{T}_{\left.\right|_{K}} & =\left(-\frac{37}{480}+\frac{1}{3} K\right) G^{3} m_{1}^{3} v_{1}^{i} v_{1}^{j} \partial_{i j}\left(\frac{1}{r_{1}}\right) \\
& +\left(\frac{19}{288}+\frac{47}{24} K\right) \frac{G^{4} m_{1}^{3} m_{2}}{r_{12}^{2}} n_{12}^{i} \partial_{i}\left(\frac{1}{r_{1}}\right)+1 \leftrightarrow 2+\left.\delta_{\xi} \hat{T}\right|_{K} .
\end{aligned}
$$

The corresponding modification of the acceleration of particle 1 is found to be

$$
\begin{aligned}
\left.\delta_{\text {Leibniz }} a_{1}^{i}\right|_{K} & =\left(\frac{37}{2}-80 K\right) \frac{G^{3} m_{2}^{3}}{c^{6} r_{12}^{4}}\left[\left(n_{12} v_{2}\right)^{2} n_{12}^{i}-\frac{1}{5} v_{2}^{2} n_{12}^{i}-\frac{2}{5}\left(n_{12} v_{2}\right) v_{2}^{i}\right] \\
& +\left(-\frac{19}{9}-\frac{188}{3} K\right) \frac{G^{4} m_{1} m_{2}^{3}}{c^{6} r_{12}^{5}} n_{12}^{i}+\left.\delta_{\xi} a_{1}^{i}\right|_{K},
\end{aligned}
$$


where the last term represents the gauge term (6.15) but computed with (6.24). Therefore, modulo a change of gauge, we see that the Leibniz modification of the acceleration brought about by the correct derivative has exactly the same form as that, given by (6.19), due to the particular one. However, we must also include the contribution of the self terms. We have redone the computation of the self terms as in Section $\nabla$ but using the $K$-dependent derivative and compared the corresponding acceleration with the previous result (6.20). We get

$$
\begin{aligned}
\left.\delta_{\text {self }} a_{1}^{i}\right|_{K}-\delta_{\text {self }} a_{1}^{i} & =\frac{G^{3} m_{2}^{3}}{c^{6} r_{12}^{4}}\left[\frac{9}{2}\left(n_{12} v_{2}\right)^{2} n_{12}^{i}-\frac{9}{10} v_{2}^{2} n_{12}^{i}-\frac{9}{5}\left(n_{12} v_{2}\right) v_{2}^{i}\right] \\
& +\left(-\frac{20}{3}+\frac{44}{3} K\right) \frac{G^{4} m_{1} m_{2}^{3}}{c^{6} r_{12}^{5}} n_{12}^{i} .
\end{aligned}
$$

Subtracting (6.19) and (6.27) for the Leibniz terms, and adding up the difference of self terms given by (6.28), we thereby obtain the difference between the total effects of the two distributional derivatives in the acceleration as

$$
\begin{aligned}
\left.\delta_{\text {distribution }} a_{1}^{i}\right|_{K}-\delta_{\text {distribution }} a_{1}^{i} & =\left(\frac{41}{2}-80 K\right) \frac{G^{3} m_{2}^{3}}{c^{6} r_{12}^{4}}\left[\left(n_{12} v_{2}\right)^{2} n_{12}^{i}-\frac{1}{5} v_{2}^{2} n_{12}^{i}-\frac{2}{5}\left(n_{12} v_{2}\right) v_{2}^{i}\right] \\
& +(1-48 K) \frac{G^{4} m_{1} m_{2}^{3}}{c^{6} r_{12}^{5}} n_{12}^{i}+\left.\delta_{\xi} a_{1}^{i}\right|_{K} .
\end{aligned}
$$

As we see, there is a dependence on the individual velocity $\mathbf{v}_{2}$ which is left out. Anticipating the result that $a_{1}^{i}$, computed with the particular derivative, is invariant under Lorentz transformations, this means that the $K$-dependent derivative breaks down the Lorentz invariance for general values of $K$ (indeed the gauge term cannot modify the behaviour under Lorentz transformations). Fortunately, we are now able to fine tune the constant $K$ so that the velocity-dependent terms in (6.29) vanish. Therefore, we obtain a unique value,

$$
K=\frac{41}{160}
$$

for which the equations of motion computed with the help of the correct derivative (2.10)(2.11) are Lorentz invariant, as they are with the particular derivative.

Thus, in the case of the correct derivative, the equations of motion are not in general Lorentz-invariant, despite the use of the Lorentzian regularization. The likely reason is that the distributional derivatives were not defined in a "Lorentzian" way (their distributional terms involve the delta-pseudo-function $\operatorname{Pf} \delta_{1}$ and not the Lorentzian one $\operatorname{Pf} \Delta_{1}$ ). Recall that the Lorentzian regularization permitted to add some crucial contributions, proportional to $m_{1}^{2} m_{2}$ in the acceleration of particle 1 [see for instance (5.35)], which are mandatory for satisfying the Lorentz invariance. In the case of the correct derivative, we find that there is still a limited class of terms, proportional to $m_{2}^{3}$, which do not obey the Lorentz invariance, unless $K$ is adjusted to the unique value (6.30). Finally we obtain 


$$
\left.\delta_{\text {distribution }} a_{1}^{i}\right|_{\frac{41}{160}}-\delta_{\text {distribution }} a_{1}^{i}=-\frac{113}{10} \frac{G^{4} m_{1} m_{2}^{3}}{c^{6} r_{12}^{5}} n_{12}^{i}+\left.\delta_{\xi} a_{1}^{i}\right|_{\frac{41}{160}} .
$$

We shall see in Section VII that the effect of the first term is simply to modify a logarithmic constant $\ln \left(r_{2}^{\prime} / s_{2}\right)$ that we shall adjust when we look for a conserved 3PN energy. After adjustement of this constant we find that the $3 \mathrm{PN}$ equations of motion computed with the two derivatives are physically the same since they merely differ by the gauge transformation appearing in 6.31).

\section{Non-distributivity contributions}

The distributive parts of the linear momentum $P_{1}^{i}$ and force $F_{1}^{i}$ densities have been written down in (3.35). They were obtained under the uncorrect hypothesis of distributivity, that is $[F G]_{1}=[F]_{1}[G]_{1}$, and we must now correct for this. (As explained in Section [II], our strategy has been to delineate as much as possible the problems, by concentrating our attention first on the computation of the regularized values of the potentials when taken individually, second on the corrections due to the non-distributivity, i.e. $[F G]_{1} \neq[F]_{1}[G]_{1}$.) Again, we find that such a subtlety as the non-distributivity makes a difference starting precisely at the $3 \mathrm{PN}$ order. We get for the required corrections in $P_{1}^{i}$ and $F_{1}^{i}$ :

$$
\begin{aligned}
& P_{1}^{i}-\left(P_{1}^{i}\right)_{\operatorname{distr}}=\frac{1}{c^{4}}\left(v_{1}^{i}\left[V^{2}\right]_{1}-v_{1}^{i}[V]_{1}^{2}\right) \\
& +\frac{1}{c^{6}}\left(12 v_{1}^{i}\left[V_{j} V_{j}\right]_{1}-12 v_{1}^{i}\left[V_{j}\right]_{1}\left[V_{j}\right]_{1}+2 v_{1}^{i}\left[V^{3}\right]_{1}\right. \\
& -3 v_{1}^{i}[V]_{1}\left[V^{2}\right]_{1}+v_{1}^{i}[V]_{1}^{3} \\
& -8\left[V_{j} \hat{W}_{i j}\right]_{1}+8\left[V_{j}\right]_{1}\left[\hat{W}_{i j}\right]_{1}+8 v_{1}^{j}\left[V \hat{W}_{i j}\right]_{1}-8 v_{1}^{j}[V]_{1}\left[\hat{W}_{i j}\right]_{1} \\
& -8\left[V^{2} V_{i}\right]_{1}+4\left[V^{2}\right]_{1}\left[V_{i}\right]_{1}+4[V]_{1}^{2}\left[V_{i}\right]_{1} \\
& \left.+\frac{1}{2} v_{1}^{2} v_{1}^{i}\left[V^{2}\right]_{1}-\frac{1}{2} v_{1}^{2} v_{1}^{i}[V]_{1}^{2}-16 v_{1}^{j}\left[V_{i} V_{j}\right]_{1}+16 v_{1}^{j}\left[V_{i}\right]_{1}\left[V_{j}\right]_{1}\right), \\
& F_{1}^{i}-\left(F_{1}^{i}\right)_{\operatorname{distr}}=\frac{1}{c^{2}}\left(-2\left[V \partial_{i} V\right]_{1}+2[V]_{1}\left[\partial_{i} V\right]_{1}\right) \\
& +\frac{1}{c^{4}}\left(-v_{1}^{2}[V]_{1}\left[\partial_{i} V\right]_{1}+v_{1}^{2}\left[V \partial_{i} V\right]_{1}-8\left[V_{j}\right]_{1}\left[\partial_{i} V_{j}\right]_{1}+8\left[V_{j} \partial_{i} V_{j}\right]_{1}\right. \\
& \left.+[V]_{1}^{2}\left[\partial_{i} V\right]_{1}-\left[V^{2}\right]_{1}\left[\partial_{i} V\right]_{1}+2\left[V^{2} \partial_{i} V\right]_{1}-2[V]_{1}\left[V \partial_{i} V\right]_{1}\right) \\
& +\frac{1}{c^{6}}\left(-\frac{1}{4} v_{1}^{4}[V]_{1}\left[\partial_{i} V\right]_{1}+\frac{1}{4} v_{1}^{4}\left[V \partial_{i} V\right]_{1}\right. \\
& +\frac{3}{2} v_{1}^{2}[V]_{1}^{2}\left[\partial_{i} V\right]_{1}-\frac{3}{2} v_{1}^{2}\left[V^{2}\right]_{1}\left[\partial_{i} V\right]_{1}+3 v_{1}^{2}\left[V^{2} \partial_{i} V\right]_{1}-3 v_{1}^{2}[V]_{1}\left[V \partial_{i} V\right]_{1}
\end{aligned}
$$




$$
\begin{aligned}
& +\frac{8}{3}[V]_{1}^{3}\left[\partial_{i} V\right]_{1}-3[V]_{1}\left[V^{2}\right]_{1}\left[\partial_{i} V\right]_{1}+\frac{2}{3}\left[V^{3}\right]_{1}\left[\partial_{i} V\right]_{1} \\
& -3[V]_{1}^{2}\left[V \partial_{i} V\right]_{1}+2\left[V^{2}\right]_{1}\left[V \partial_{i} V\right]_{1}+2[V]_{1}\left[V^{2} \partial_{i} V\right]_{1}-\frac{4}{3}\left[V^{3} \partial_{i} V\right]_{1} \\
& -16\left[\partial_{i} V_{j}\right]_{1}\left[\hat{R}_{j}\right]_{1}+16\left[\partial_{i} V_{j} \hat{R}_{j}\right]_{1}-16\left[V_{j}\right]_{1}\left[\partial_{i} \hat{R}_{j}\right]_{1}+16\left[V_{j} \partial_{i} \hat{R}_{j}\right]_{1} \\
& +8[V]_{1}\left[\partial_{i} V_{j}\right]_{1}\left[V_{j}\right]_{1}+8[V]_{1}\left[\partial_{i} V_{j} V_{j}\right]_{1}-16\left[V \partial_{i} V_{j} V_{j}\right]_{1} \\
& +4\left[\partial_{i} V\right]_{1}\left[V_{j}\right]_{1}\left[V_{j}\right]_{1}+4\left[\partial_{i} V\right]_{1}\left[V_{j} V_{j}\right]_{1}-8\left[\partial_{i} V V_{j} V_{j}\right]_{1} \\
& -12 v_{1}^{2}\left[\partial_{i} V_{j}\right]_{1}\left[V_{j}\right]_{1}+12 v_{1}^{2}\left[\partial_{i} V_{j} V_{j}\right]_{1} \\
& +4 v_{1}^{j}[V]_{1}^{2}\left[\partial_{i} V_{j}\right]_{1}+4 v_{1}^{j}\left[V^{2}\right]_{1}\left[\partial_{i} V_{j}\right]_{1}-8 v_{1}^{j}\left[V^{2} \partial_{i} V_{j}\right]_{1} \\
& +8 v_{1}^{j}[V]_{1}\left[\partial_{i} V\right]_{1}\left[V_{j}\right]_{1}+8 v_{1}^{j}\left[V \partial_{i} V\right]_{1}\left[V_{j}\right]_{1}-16 v_{1}^{j}\left[V \partial_{i} V V_{j}\right]_{1} \\
& +8 v_{1}^{j}\left[V_{k}\right]_{1}\left[\partial_{i} \hat{W}_{j k}\right]_{1}-8 v_{1}^{j}\left[V_{k} \partial_{i} \hat{W}_{j k}\right]_{1}+8 v_{1}^{j}\left[\partial_{i} V_{k}\right]_{1}\left[\hat{W}_{j k}\right]_{1}-8 v_{1}^{j}\left[\partial_{i} V_{k} \hat{W}_{j k}\right]_{1} \\
& -4 v_{1}^{j} v_{1}^{k}[V]_{1}\left[\partial_{i} \hat{W}_{j k}\right]_{1}+4 v_{1}^{j} v_{1}^{k}\left[V \partial_{i} \hat{W}_{j k}\right]_{1} \\
& -4 v_{1}^{j} v_{1}^{k}\left[\partial_{i} V\right]_{1}\left[\hat{W}_{j k}\right]_{1}+4 v_{1}^{j} v_{1}^{k}\left[\partial_{i} V \hat{W}_{j k}\right]_{1} \\
& +16 v_{1}^{j} v_{1}^{k}\left[\partial_{i} V_{k}\right]_{1}\left[V_{j}\right]_{1}-16 v_{1}^{j} v_{1}^{k}\left[\partial_{i} V_{k} V_{j}\right]_{1} \\
& \left.+8[\hat{X}]_{1}\left[\partial_{i} V\right]_{1}-8\left[\hat{X} \partial_{i} V\right]_{1}+8[V]_{1}\left[\partial_{i} \hat{X}\right]_{1}-8\left[V \partial_{i} \hat{X}\right]_{1}\right) \text {. }
\end{aligned}
$$

These formulas look complicated but are in fact rather simple to evaluate because they require only some lower-order post-Newtonian precision in the potentials, with notably all the difficult non-compact potentials needed at the Newtonian order only (hence the interest of separating out the problems as we did). Note that it is crucial here to employ the Lorentzian regularization $[F]_{1}$. The net result of this computation is

$$
\begin{aligned}
P_{1}^{i}-\left(P_{1}^{i}\right)_{\mathrm{distr}}= & \frac{G^{3} m_{1}^{2} m_{2}}{c^{6} r_{12}^{3}}\left(\frac{2}{5}\left(n_{12} v_{12}\right) n_{12}^{i}-\frac{2}{15} v_{12}^{i}\right) \\
F_{1}^{i}-\left(F_{1}^{i}\right)_{\mathrm{distr}}= & \frac{G^{3} m_{1}^{2} m_{2}}{c^{6} r_{12}^{4}}\left(\left[\frac{241}{70} \frac{G m_{1}}{r_{12}}-\frac{51}{70} \frac{G m_{2}}{r_{12}}\right] n_{12}^{i}\right. \\
& \left.+\frac{723}{28}\left(n_{12} v_{12}\right)^{2} n_{12}^{i}-\frac{723}{140} v_{12}^{2} n_{12}^{i}-\frac{723}{70}\left(n_{12} v_{12}\right) v_{12}^{i}\right) .
\end{aligned}
$$

Therefore the supplement of acceleration linked to the non-distributivity is

$$
\begin{aligned}
a_{1}^{i}-\left(a_{1}^{i}\right)_{\text {distr }} & =\frac{G^{3} m_{1}^{2} m_{2}}{c^{6} r_{12}^{4}}\left(\left[\frac{779}{210} \frac{G m_{1}}{r_{12}}-\frac{97}{210} \frac{G m_{2}}{r_{12}}\right] n_{12}^{i}\right. \\
& \left.+\frac{779}{28}\left(n_{12} v_{12}\right)^{2} n_{12}^{i}-\frac{779}{140} v_{12}^{2} n_{12}^{i}-\frac{779}{70}\left(n_{12} v_{12}\right) v_{12}^{i}\right)
\end{aligned}
$$

Since only the relative velocity $\mathbf{v}_{12}$ is involved this part of the acceleration is Galilean invariant. Furthermore, it can be expressed in a simpler way by introducing an infinitesimal gauge transformation of the type (6.11). We pose

$$
\left(\epsilon_{1}\right)_{\mathrm{distr}}=-\frac{779}{420} \frac{m_{1} m_{2}^{2}}{m^{3}}
$$




$$
\left(\epsilon_{1}\right)_{\mathrm{distr}}=-\frac{779}{420} \frac{m_{1}^{2} m_{2}}{m^{3}}
$$

and easily obtain

$$
a_{1}^{i}-\left(a_{1}^{i}\right)_{\mathrm{distr}}=\left(\delta_{\xi} a_{1}^{i}\right)_{\mathrm{distr}}+\frac{G^{4} m_{1} m_{2}^{2}}{c^{6} r_{12}^{5}}\left[-\frac{97}{210} m_{1}+\frac{779}{210} m_{2}\right] n_{12}^{i} .
$$

Thus, the only Physics brought about by the non-distributivity (i.e. which is not affected by a gauge transformation) is constituted by the quartic $\left(G^{4}\right)$ term displayed in the right-hand side of $(6.36)$.

\section{THE 3PN EQUATIONS OF MOTION}

\section{A. Existence of the conserved energy}

At present, the equations of motion are complete. We want now to look for the conserved energy associated with these equations at the 3PN order (considering of course only the conservative part of the equations, i.e. excluding the radiation reaction acceleration at the 2.5PN order). We shall see that the existence of an energy is not immediate, but requires the adjustment of a certain constant.

We proved in Section $\square$ that the equations of motion of body 1 depend on two arbitrary constants, which are the constant $r_{1}^{\prime}$, tending to zero as we approach the particle 1 (but considered here as taking some finite non-zero value), and the constant $s_{2}$ associated with the Hadamard regularization near the other particle 2 [see (2.3)]. Similarly, the equations of body 2 depend on the constants $r_{2}^{\prime}$ and $s_{1}$. All these constants appear inside the logarithms entering the equations of motion in harmonic coordinates. Gathering the results for the "logarithmic" part of the equations of body 1, we obtain

$$
\begin{aligned}
a_{1}^{i} & =\frac{44}{3} \frac{G^{4} m_{1}^{3} m_{2}}{c^{6} r_{12}^{5}} n_{12}^{i} \ln \left(\frac{r_{12}}{r_{1}^{\prime}}\right)-\frac{44}{3} \frac{G^{4} m_{1} m_{2}^{3}}{c^{6} r_{12}^{5}} n_{12}^{i} \ln \left(\frac{r_{12}}{s_{2}}\right) \\
& +\frac{G^{3} m_{1}^{2} m_{2}}{c^{6} r_{12}^{4}}\left[110\left(n_{12} v_{12}\right)^{2} n_{12}^{i}-22 v_{12}^{2} n_{12}^{i}-44\left(n_{12} v_{12}\right) v_{12}^{i}\right] \ln \left(\frac{r_{12}}{r_{1}^{\prime}}\right)+\ldots,
\end{aligned}
$$

where the dots indicate the terms which do not contain any logarithms. The terms shown in (7.1) contain the whole dependence of the acceleration of 1 over $r_{1}^{\prime}$ and $s_{2}$; there are no other constants elsewhere. Notice that $s_{2}$ enters a single quartic-order term proportional to $G^{4} m_{1} m_{2}^{3}$. Now, most of the terms in $(7.1)$ can in fact be gauged away. To see this, we apply the formula (6.15) with the particular choice

$$
\begin{aligned}
& \left(\epsilon_{1}\right)_{\ln }=-\frac{22}{3} \frac{m_{1} m_{2}^{2}}{m^{3}} \ln \left(\frac{r_{12}}{r_{2}^{\prime}}\right), \\
& \left(\epsilon_{2}\right)_{\ln }=-\frac{22}{3} \frac{m_{1}^{2} m_{2}}{m^{3}} \ln \left(\frac{r_{12}}{r_{1}^{\prime}}\right) .
\end{aligned}
$$


The corresponding transformation of the acceleration is $(6.15)$, except that $\left(\epsilon_{1}\right)_{\ln }$ and $\left(\epsilon_{2}\right)_{\ln }$ depend on time through the orbital separation $r_{12}$, so in fact this formula should contain also some terms proportional to the time-derivatives of $\left(\epsilon_{1}\right)_{\ln }$ and $\left(\epsilon_{2}\right)_{\ln }$; but the point for us is that these extra terms are free of any logarithms. Therefore, modulo the dots indicating the logarithmic-free terms, we can write

$$
a_{1}^{i}=\left(\delta_{\xi} a_{1}^{i}\right)_{\ln }-\frac{44}{3} \frac{G^{4} m_{1} m_{2}^{3}}{c^{6} r_{12}^{5}} n_{12}^{i} \ln \left(\frac{r_{2}^{\prime}}{s_{2}}\right)+\ldots
$$

where $\left(\delta_{\xi} a_{1}^{i}\right)_{\text {ln }}$ denotes the coordinate change of the acceleration.

The term in (7.3) which is left out after this coordinate change depends only on the ratio between $r_{2}^{\prime}$ and $s_{2}$ (similarly, in the equations of motion of body 2, we would find the ratio of $r_{1}^{\prime}$ and $s_{1}$ ). This term is of the same type as the one in (6.31) giving the difference of accelerations, modulo a change of gauge, when different distributional derivatives are used. Notice that the constant $r_{2}^{\prime}$ was originally absent from the equations of motion of 1 , but has to be introduced in order to "remove" these logarithms by the coordinate transformation. Therefore, the only physical freedom remaining in the equations of motion is the yet unspecified constant $\ln \left(\frac{r_{2}^{\prime}}{s_{2}}\right)$. Now we use this freedom to find a conserved energy associated with the equations of motion, which means a local-in-time functional $E$ of the trajectories and velocities of the two particles, i.e.

$$
E=E\left[\mathbf{y}_{1}(t), \mathbf{y}_{2}(t) ; \mathbf{v}_{1}(t), \mathbf{v}_{2}(t)\right]
$$

which is constant as a consequence of the $3 \mathrm{PN}$ equations of motion, i.e.

$$
\frac{d E}{d t} \equiv v_{1}^{i} \frac{\partial E}{\partial y_{1}^{i}}+v_{2}^{i} \frac{\partial E}{\partial y_{2}^{i}}+a_{1}^{i} \frac{\partial E}{\partial v_{1}^{i}}+a_{2}^{i} \frac{\partial E}{\partial v_{2}^{i}}=\overline{\mathcal{O}}(7)
$$

The accelerations $\mathbf{a}_{1}$ and $\mathbf{a}_{1}$ are to be replaced by the functionals of the positions and velocities given by the $3 \mathrm{PN}$ equations of motion. Our special notation for the remainder means a radiation-reaction term which is purely of order $2.5 \mathrm{PN}$ plus the neglected terms at 3.5PN; schematically $\overline{\mathcal{O}}(7)=\frac{1}{c^{5}} F_{5}+\mathcal{O}(7)$. See (7.18) below for the expression of the term $\frac{1}{c^{5}} F_{5}$. If an energy exists, the quantity $m_{1} a_{1}^{i} v_{1}^{i}+m_{2} a_{2}^{i} v_{2}^{i}$ must be a total time derivative. In practive, we look for a local-in-time functional $D\left[\mathbf{y}_{1}, \mathbf{y}_{2} ; \mathbf{v}_{1}, \mathbf{v}_{2}\right]$ such that

$$
m_{1} a_{1}^{i} v_{1}^{i}+m_{2} a_{2}^{i} v_{2}^{i}+\frac{d D}{d t}=\overline{\mathcal{O}}(7)
$$

and we obtain the energy as $E=\frac{1}{2} m_{1} \mathbf{v}_{1}^{2}+\frac{1}{2} m_{2} \mathbf{v}_{2}^{2}+D$. Now, the computation with our 3PN equations of motion (obtained by means of, say, the particular derivative) shows that the quantity $D$ does not exist for any values of the constants $\ln \left(r_{2}^{\prime} / s_{2}\right)$ and $\ln \left(r_{1}^{\prime} / s_{1}\right)$. However, we find that this "nearly" works, because we can determine some $\hat{D}$ such that 


$$
m_{1} a_{1}^{i} v_{1}^{i}+m_{2} a_{2}^{i} v_{2}^{i}+\frac{d \hat{D}}{d t}=-\frac{44}{3} \frac{G^{4} m_{1}^{2} m_{2}^{2}}{c^{6} r_{12}^{5}}\left\{m_{2}\left(n_{12} v_{1}\right)\left[\ln \left(\frac{r_{2}^{\prime}}{s_{2}}\right)-\frac{159}{308}\right]+1 \leftrightarrow 2\right\}+\overline{\mathcal{O}}(7)
$$

From the computation we obtain $\hat{D}$ as a well-defined local functional of the positions and velocities of the particules [containing in particular some logarithms $\ln \left(r_{12} / r_{1}^{\prime}\right)$ and $\left.\ln \left(r_{12} / r_{2}^{\prime}\right)\right]$. The right-hand side of (7.7) cannot be written, for generic values of $\ln \left(r_{2}^{\prime} / s_{2}\right)$ and $\ln \left(r_{1}^{\prime} / s_{1}\right)$, in the form of a total time-derivative. It would be possible, for this to be the case, to adopt the simplest choice that both these constants are numerically equal to $\frac{159}{308}$. However, this choice does not represent the most general solution for obtaining a total time-derivative. Indeed, nothing prevents $\ln \left(r_{1}^{\prime} / s_{1}\right)$ and $\ln \left(r_{2}^{\prime} / s_{2}\right)$ to depend also on the masses $m_{1}$ and $m_{2}$, and therefore such a dependence on the masses should in fact be mandatory (totalitarian principle). Since the regularization procedure followed in this paper is more mathematical than physical, we can be confident that no Physics will be overlooked only if at each step we obtain the most general solution allowed by the process. Unfortunately, the most general solution in this case contains an arbitrary parameter.

The necessary and sufficient condition for the right side of (7.7) to be a total timederivative is that the factor of $\left(n_{12} v_{1}\right)$ in (7.7) be invariant by exchanging the particle's labels 2 and 1, i.e.

$$
m_{2}\left[\ln \left(\frac{r_{2}^{\prime}}{s_{2}}\right)-\frac{159}{308}\right]=m_{1}\left[\ln \left(\frac{r_{1}^{\prime}}{s_{1}}\right)-\frac{159}{308}\right] .
$$

Denoting by $\lambda m$ the common value of both sides of (7.8), where $\lambda$ is a constant and $m=$ $m_{1}+m_{2}$, we obtain the most general solution as

$$
\begin{aligned}
& \ln \left(\frac{r_{2}^{\prime}}{s_{2}}\right)=\frac{159}{308}+\lambda \frac{m}{m_{2}}, \\
& \ln \left(\frac{r_{1}^{\prime}}{s_{1}}\right)=\frac{159}{308}+\lambda \frac{m}{m_{1}} .
\end{aligned}
$$

This $\lambda$ is a dimensionless quantity which is the same for the two particles 1 and 2 . We now prove that $\lambda$ is necessarily a pure numerical constant, independent of the masses. Notice that the $\lambda$-term in (7.9a) will yield a contribution to the acceleration of 1 which is, as concerns the dependence over the masses, of the type $m_{1} m_{2}^{2} m \lambda$ [see (7.3)]. If $\lambda$ depends on the masses, it must be a symmetric function of $m_{1}$ and $m_{2}$, and therefore it can be expressed solely in terms of the symmetric mass ratio $\nu=\frac{m_{1} m_{2}}{m^{2}}$. Suppose that $\lambda=\sum_{-\infty}^{+\infty} \lambda_{i} \nu^{i}$, where the $\lambda_{i}{ }^{\prime} \mathrm{s}$ are numerical constants, so the $\lambda$-term in the acceleration of 1 is of the type $m_{1} m_{2}^{2} m \sum \lambda_{i} \nu^{i}$. First, we see that all the cases $i \leq-1$ are excluded because the equations of motion would not have the correct perturbative limit when $\nu \rightarrow 0$; for instance, in the case $i=-1$, we get a term of the type $m^{3} m_{2}$ which tends to $m_{2}^{4}$ in this limit, and therefore modifies the geodesic 
motion of a test particle around a Schwarzschild black hole, which is of course excluded. Second, the cases $i \geq 1$, though they pass the simplest physical requirements, imply that the individual particle accelerations are no longer polynomials in the two individual masses $m_{1}$ and $m_{2}$, because of the appearance of inverse powers of the total mass $m=m_{1}+m_{2}$. For instance, the case $i=1$ leads to a term of the type $\frac{m_{1}^{2} m_{2}^{3}}{m}$. But we know that when doing a diagrammatic expansion of the $N$ body problem based on the post-Minkowskian expansion (see [59] for the details of the method) that each successive diagram is a polynomial of the $N$ masses. Therefore, we exclude the possibility that some inverse powers of the total mass appear, and find, in conclusion, that $\lambda$ is a pure constant $\left(\lambda=\lambda_{0}\right)$.

At last, we have succeeded in finding a conserved energy at the 3PN order by specifying an unknown logarithmic ratio, but at the price of having introduced an arbitrary purely numerical constant $\lambda$. The constant $\lambda$ will be left undetermined in the present work. So the final 3PN equations of motion we obtain in this paper, as well as the final 3PN energy, depend on the unknown parameter $\lambda$. The appearance of $\lambda$ suggests that the present formalism, based on a point-mass regularization, is physically incomplete. The resulting ambiguity is equivalent to the "static" ambiguity found by Jaranowski and Schäfer [33]. It is probably linked to the fact that one can write the Einstein field equations into many different forms, which are all equivalent in the case of regular sources, but which are in general not equivalent in the case of point-particles because the distributional derivative does not obey the Leibniz rule. If we had chosen initially a different form of the field equations, the Leibniz terms we computed in Section VI, could have been different. More precisely, only that part of the Leibniz terms which is Galilean-invariant and consequently is not required by the Lorentzinvariance symmetry could change. But we have seen in (6.19) and (6.31) that the Galileaninvariant part of the Leibniz terms is precisely made of only one term, which is of the same type (proportional to $\left.G^{4} m_{1} m_{2}^{3}\right)$ as the term containing the constant $\ln \left(r_{2}^{\prime} / s_{2}\right)$ [see (7.3)] that we have adjusted in the equations (7.9a), resulting in the appearance of the constant $\lambda$. Thus, in agreement with Jaranowski and Schäfer [33], we might say that $\lambda$ encodes an ambiguity associated with the violation of the Leibniz rule by the distributional derivative. At a deeper level, this would mean that the ambiguity is a consequence of a theorem of Schwartz [60] according to which it is impossible to define at once a multiplication of distributions which agrees with the ordinary product for continuous functions, and a derivation of distributions which satisfies the Leibniz rule and reduces to the ordinary derivative in the case of $C^{1}$ functions. If this explanation is correct, it is unlikely that the constant $\lambda$ could be determined within the present formalism.

We find by combining (7.9) and (7.7) that the dependence of the 3PN energy $E$ on $\lambda$ is

$$
E=\hat{E}-\frac{11}{3} \lambda \frac{G^{4} m_{1}^{2} m_{2}^{2} m}{c^{6} r_{12}^{4}},
$$

where $\hat{E}$ does not depend on $\lambda$, while we obtain, using (7.3), that the acceleration writes 


$$
a_{1}^{i}=\hat{a}_{1}^{i}-\frac{44}{3} \lambda \frac{G^{4} m_{1} m_{2}^{2} m}{c^{6} r_{12}^{5}} n_{12}^{i},
$$

where similarly $\hat{a}_{1}^{i}$ is independent of $\lambda$. On the other hand, the acceleration and energy depend also on the two constants $r_{1}^{\prime}$ and $r_{2}^{\prime}$, but from the previous discussion this is not a problem because $r_{1}^{\prime}$ and $r_{2}^{\prime}$ are associated with an arbitrariness in the choice of coordinates: the $3 \mathrm{PN}$ equations of motion contain the logarithms $\ln \left(r_{12} / r_{1}^{\prime}\right)$ and $\ln \left(r_{12} / r_{2}^{\prime}\right)$ which have been shown in (7.1) to be in the form of the gauge transformation associated with (7.2). In particular, the "constants" $\ln r_{1}^{\prime}$ and $\ln r_{2}^{\prime}$, which might be said to be formally infinite because $r_{1}^{\prime}$ and $r_{2}^{\prime}$ were tending to zero [recall the discussion after (5.18)], will never appear in any physical result. Similarly, the dependence of the energy on the logarithms $\ln \left(r_{12} / r_{1}^{\prime}\right)$ and $\ln \left(r_{12} / r_{2}^{\prime}\right)$ is pure gauge. From (6.16) we get

$$
E=\frac{22}{3} \frac{G^{3} m_{1}^{3} m_{2}}{c^{6} r_{12}^{3}}\left[-\frac{G m_{2}}{r_{12}}+3\left(n_{12} v_{1}\right)\left(n_{12} v_{12}\right)-\left(v_{1} v_{12}\right)\right] \ln \left(\frac{r_{12}}{r_{1}^{\prime}}\right)+1 \leftrightarrow 2+\ldots
$$

where the dots denote the terms independent of logarithms [this result can also be checked directly using (7.1)].

Finally, to be more specific about the influence of the distributional derivative, notice that the solution we have obtained in (7.9) corresponds to the "particular" distributional derivative defined by (2.9). If one uses the "correct" derivative (2.10)-(2.11) instead, with the value $K=\frac{41}{160}$ we have obtained in (6.30) from the Lorentz invariance, we obtain the same equation to be solved as (7.7) but with the rational fraction $+\frac{783}{3080}$ instead of $-\frac{159}{308}$. This is easily seen thanks to (6.31). So, the solution becomes in this case

$$
\begin{aligned}
& \ln \left(\frac{r_{2}^{\prime}}{s_{2}}\right)=-\frac{783}{3080}+\lambda \frac{m}{m_{2}}, \\
& \ln \left(\frac{r_{1}^{\prime}}{s_{1}}\right)=-\frac{783}{3080}+\lambda \frac{m}{m_{1}} .
\end{aligned}
$$

Replacing this into the equations of motion (and associated energy), it is then clear that they are physically the same as those computed with the other derivative, because they differ by the mere change of gauge,

$$
\left.a_{1}^{i}\right|_{\frac{41}{160}}-a_{1}^{i}=\left.\delta_{\xi} a_{1}^{i}\right|_{\frac{41}{160}}
$$

that we obtained in (6.31). We give it here thoroughtly for completeness:

$$
\begin{aligned}
\left.\delta_{\xi} a_{1}^{i}\right|_{\frac{41}{160}} & =\frac{13}{25} \frac{G^{4} m_{1} m_{2}}{c^{6} r_{12}^{5}}\left(m_{1}^{2}-m_{2}^{2}\right) n_{12}^{i} \\
& +\frac{13}{50} \frac{G^{3} m_{2}^{3}}{c^{6} r_{12}^{4}}\left[-15\left(n_{12} v_{12}\right)^{2} n_{12}^{i}+3 v_{12}^{2} n_{12}^{i}+6\left(n_{12} v_{12}\right) v_{12}^{i}\right] .
\end{aligned}
$$




\section{B. End results}

We present the 3PN equations of motion of the particle 1 in harmonic coordinates, which are obtained by summing up all the contributions of the potentials computed in Sections IV and $\mathrm{V}$, as well as the pieces due to the non-distributivity and the Leibniz terms (see Section VI). The equations depend on two gauge constants $r_{1}^{\prime}$ and $r_{2}^{\prime}$ through some logarithms, and on one unknown purely numerical coefficient $\lambda$. The equations of the particle 2 are obtained by exchanging all the labels $1 \leftrightarrow 2$.

$$
\begin{aligned}
& a_{1}^{i}=-\frac{G m_{2} n_{12}^{i}}{r_{12}^{2}} \\
& +\frac{1}{c^{2}}\left\{\left[\frac{5 G^{2} m_{1} m_{2}}{r_{12}^{3}}+\frac{4 G^{2} m_{2}^{2}}{r_{12}^{3}}+\frac{G m_{2}}{r_{12}^{2}}\left(\frac{3}{2}\left(n_{12} v_{2}\right)^{2}-v_{1}^{2}+4\left(v_{1} v_{2}\right)-2 v_{2}^{2}\right)\right] n_{12}^{i}\right. \\
& \left.+\frac{G m_{2}}{r_{12}^{2}}\left(4\left(n_{12} v_{1}\right)-3\left(n_{12} v_{2}\right)\right)\left(v_{1}^{i}-v_{2}^{i}\right)\right\} \\
& +\frac{1}{c^{4}}\left\{\left[-\frac{57 G^{3} m_{1}^{2} m_{2}}{4 r_{12}^{4}}-\frac{69 G^{3} m_{1} m_{2}^{2}}{2 r_{12}^{4}}-\frac{9 G^{3} m_{2}^{3}}{r_{12}^{4}}+\frac{G m_{2}}{r_{12}^{2}}\left(-\frac{15}{8}\left(n_{12} v_{2}\right)^{4}+\frac{3}{2}\left(n_{12} v_{2}\right)^{2} v_{1}^{2}\right.\right.\right. \\
& \left.-6\left(n_{12} v_{2}\right)^{2}\left(v_{1} v_{2}\right)-2\left(v_{1} v_{2}\right)^{2}+\frac{9}{2}\left(n_{12} v_{2}\right)^{2} v_{2}^{2}+4\left(v_{1} v_{2}\right) v_{2}^{2}-2 v_{2}^{4}\right) \\
& +\frac{G^{2} m_{1} m_{2}}{r_{12}^{3}}\left(\frac{39}{2}\left(n_{12} v_{1}\right)^{2}-39\left(n_{12} v_{1}\right)\left(n_{12} v_{2}\right)+\frac{17}{2}\left(n_{12} v_{2}\right)^{2}-\frac{15}{4} v_{1}^{2}-\frac{5}{2}\left(v_{1} v_{2}\right)\right. \\
& \left.\left.+\frac{5}{4} v_{2}^{2}\right)+\frac{G^{2} m_{2}^{2}}{r_{12}^{3}}\left(2\left(n_{12} v_{1}\right)^{2}-4\left(n_{12} v_{1}\right)\left(n_{12} v_{2}\right)-6\left(n_{12} v_{2}\right)^{2}-8\left(v_{1} v_{2}\right)+4 v_{2}^{2}\right)\right] n_{12}^{i} \\
& +\left[\frac{G^{2} m_{2}^{2}}{r_{12}^{3}}\left(-2\left(n_{12} v_{1}\right)-2\left(n_{12} v_{2}\right)\right)+\frac{G^{2} m_{1} m_{2}}{r_{12}^{3}}\left(-\frac{63}{4}\left(n_{12} v_{1}\right)+\frac{55}{4}\left(n_{12} v_{2}\right)\right)\right. \\
& +\frac{G m_{2}}{r_{12}^{2}}\left(-6\left(n_{12} v_{1}\right)\left(n_{12} v_{2}\right)^{2}+\frac{9}{2}\left(n_{12} v_{2}\right)^{3}+\left(n_{12} v_{2}\right) v_{1}^{2}-4\left(n_{12} v_{1}\right)\left(v_{1} v_{2}\right)\right. \\
& \left.\left.\left.+4\left(n_{12} v_{2}\right)\left(v_{1} v_{2}\right)+4\left(n_{12} v_{1}\right) v_{2}^{2}-5\left(n_{12} v_{2}\right) v_{2}^{2}\right)\right]\left(v_{1}^{i}-v_{2}^{i}\right)\right\} \\
& +\frac{1}{c^{5}}\left\{\left[\frac{208 G^{3} m_{1} m_{2}^{2}}{15 r_{12}^{4}}\left(\left(n_{12} v_{1}\right)-\left(n_{12} v_{2}\right)\right)-\frac{24 G^{3} m_{1}^{2} m_{2}}{5 r_{12}^{4}}\left(\left(n_{12} v_{1}\right)-\left(n_{12} v_{2}\right)\right)\right.\right. \\
& \left.+\frac{12 G^{2} m_{1} m_{2}}{5 r_{12}^{3}}\left(\left(n_{12} v_{1}\right)-\left(n_{12} v_{2}\right)\right)\left[v_{1}^{2}-2\left(v_{1} v_{2}\right)+v_{2}^{2}\right]\right] n_{12}^{i}+\left[\frac{8 G^{3} m_{1}^{2} m_{2}}{5 r_{12}^{4}}\right. \\
& \left.\left.-\frac{32 G^{3} m_{1} m_{2}^{2}}{5 r_{12}^{4}}-\frac{4 G^{2} m_{1} m_{2}}{5 r_{12}^{3}}\left[v_{1}^{2}-2\left(v_{1} v_{2}\right)+v_{2}^{2}\right]\right]\left(v_{1}^{i}-v_{2}^{i}\right)\right\} \\
& +\frac{1}{c^{6}}\left\{\left[\frac { G m _ { 2 } } { r _ { 1 2 } ^ { 2 } } \left(\frac{35}{16}\left(n_{12} v_{2}\right)^{6}-\frac{15}{8}\left(n_{12} v_{2}\right)^{4} v_{1}^{2}+\frac{15}{2}\left(n_{12} v_{2}\right)^{4}\left(v_{1} v_{2}\right)+3\left(n_{12} v_{2}\right)^{2}\left(v_{1} v_{2}\right)^{2}\right.\right.\right.
\end{aligned}
$$




$$
\begin{aligned}
& -\frac{15}{2}\left(n_{12} v_{2}\right)^{4} v_{2}^{2}+\frac{3}{2}\left(n_{12} v_{2}\right)^{2} v_{1}^{2} v_{2}^{2}-12\left(n_{12} v_{2}\right)^{2}\left(v_{1} v_{2}\right) v_{2}^{2}-2\left(v_{1} v_{2}\right)^{2} v_{2}^{2} \\
& \left.+\frac{15}{2}\left(n_{12} v_{2}\right)^{2} v_{2}^{4}+4\left(v_{1} v_{2}\right) v_{2}^{4}-2 v_{2}^{6}\right)+\frac{G^{2} m_{1} m_{2}}{r_{12}^{3}}\left(-\frac{171}{8}\left(n_{12} v_{1}\right)^{4}\right. \\
& +\frac{171}{2}\left(n_{12} v_{1}\right)^{3}\left(n_{12} v_{2}\right)-\frac{723}{4}\left(n_{12} v_{1}\right)^{2}\left(n_{12} v_{2}\right)^{2}+\frac{383}{2}\left(n_{12} v_{1}\right)\left(n_{12} v_{2}\right)^{3} \\
& -\frac{455}{8}\left(n_{12} v_{2}\right)^{4}+\frac{229}{4}\left(n_{12} v_{1}\right)^{2} v_{1}^{2}-\frac{205}{2}\left(n_{12} v_{1}\right)\left(n_{12} v_{2}\right) v_{1}^{2}+\frac{191}{4}\left(n_{12} v_{2}\right)^{2} v_{1}^{2}-\frac{91}{8} v_{1}^{4} \\
& -\frac{229}{2}\left(n_{12} v_{1}\right)^{2}\left(v_{1} v_{2}\right)+244\left(n_{12} v_{1}\right)\left(n_{12} v_{2}\right)\left(v_{1} v_{2}\right)-\frac{225}{2}\left(n_{12} v_{2}\right)^{2}\left(v_{1} v_{2}\right) \\
& +\frac{91}{2} v_{1}^{2}\left(v_{1} v_{2}\right)-\frac{177}{4}\left(v_{1} v_{2}\right)^{2}+\frac{229}{4}\left(n_{12} v_{1}\right)^{2} v_{2}^{2}-\frac{283}{2}\left(n_{12} v_{1}\right)\left(n_{12} v_{2}\right) v_{2}^{2} \\
& \left.+\frac{259}{4}\left(n_{12} v_{2}\right)^{2} v_{2}^{2}-\frac{91}{4} v_{1}^{2} v_{2}^{2}+43\left(v_{1} v_{2}\right) v_{2}^{2}-\frac{81}{8} v_{2}^{4}\right)+\frac{G^{2} m_{2}^{2}}{r_{12}^{3}}\left(-6\left(n_{12} v_{1}\right)^{2}\left(n_{12} v_{2}\right)^{2}\right. \\
& +12\left(n_{12} v_{1}\right)\left(n_{12} v_{2}\right)^{3}+6\left(n_{12} v_{2}\right)^{4}+4\left(n_{12} v_{1}\right)\left(n_{12} v_{2}\right)\left(v_{1} v_{2}\right)+12\left(n_{12} v_{2}\right)^{2}\left(v_{1} v_{2}\right) \\
& \left.+4\left(v_{1} v_{2}\right)^{2}-4\left(n_{12} v_{1}\right)\left(n_{12} v_{2}\right) v_{2}^{2}-12\left(n_{12} v_{2}\right)^{2} v_{2}^{2}-8\left(v_{1} v_{2}\right) v_{2}^{2}+4 v_{2}^{4}\right) \\
& +\frac{G^{3} m_{2}^{3}}{r_{12}^{4}}\left(-\left(n_{12} v_{1}\right)^{2}+2\left(n_{12} v_{1}\right)\left(n_{12} v_{2}\right)+\frac{43}{2}\left(n_{12} v_{2}\right)^{2}+18\left(v_{1} v_{2}\right)-9 v_{2}^{2}\right) \\
& +\frac{G^{3} m_{1} m_{2}^{2}}{r_{12}^{4}}\left(\frac{415}{8}\left(n_{12} v_{1}\right)^{2}-\frac{375}{4}\left(n_{12} v_{1}\right)\left(n_{12} v_{2}\right)+\frac{1113}{8}\left(n_{12} v_{2}\right)^{2}\right. \\
& \left.-\frac{615}{64}\left(\left(n_{12} v_{1}\right)-\left(n_{12} v_{2}\right)\right)^{2} \pi^{2}+18 v_{1}^{2}+\frac{123}{64} \pi^{2}\left(v_{1}-v_{2}\right)^{2}+33\left(v_{1} v_{2}\right)-\frac{33}{2} v_{2}^{2}\right) \\
& +\frac{G^{3} m_{1}^{2} m_{2}}{r_{12}^{4}}\left(-\frac{45887}{168}\left(n_{12} v_{1}\right)^{2}+\frac{24025}{42}\left(n_{12} v_{1}\right)\left(n_{12} v_{2}\right)-\frac{10469}{42}\left(n_{12} v_{2}\right)^{2}\right. \\
& +\frac{48197}{840} v_{1}^{2}-\frac{36227}{420}\left(v_{1} v_{2}\right)+\frac{36227}{840} v_{2}^{2}+110\left(\left(n_{12} v_{1}\right)-\left(n_{12} v_{2}\right)\right)^{2} \ln \left(\frac{r_{12}}{r_{1}^{\prime}}\right) \\
& \left.-22\left(v_{1}-v_{2}\right)^{2} \ln \left(\frac{r_{12}}{r_{1}^{\prime}}\right)\right)+\frac{16 G^{4} m_{2}^{4}}{r_{12}^{5}}+\frac{G^{4} m_{1}^{2} m_{2}^{2}}{r_{12}^{5}}\left(\frac{34763}{210}-\frac{44 \lambda}{3}-\frac{41}{16} \pi^{2}\right) \\
& +\frac{G^{4} m_{1}^{3} m_{2}}{r_{12}^{5}}\left(-\frac{3187}{1260}+\frac{44}{3} \ln \left(\frac{r_{12}}{r_{1}^{\prime}}\right)\right)+\frac{G^{4} m_{1} m_{2}^{3}}{r_{12}^{5}}\left(\frac{10478}{63}-\frac{44 \lambda}{3}-\frac{41}{16} \pi^{2}\right. \\
& \left.\left.-\frac{44}{3} \ln \left(\frac{r_{12}}{r_{2}^{\prime}}\right)\right)\right] n_{12}^{i}+\left[\frac { G m _ { 2 } } { r _ { 1 2 } ^ { 2 } } \left(\frac{15}{2}\left(n_{12} v_{1}\right)\left(n_{12} v_{2}\right)^{4}-\frac{45}{8}\left(n_{12} v_{2}\right)^{5}-\frac{3}{2}\left(n_{12} v_{2}\right)^{3} v_{1}^{2}\right.\right. \\
& +6\left(n_{12} v_{1}\right)\left(n_{12} v_{2}\right)^{2}\left(v_{1} v_{2}\right)-6\left(n_{12} v_{2}\right)^{3}\left(v_{1} v_{2}\right)-2\left(n_{12} v_{2}\right)\left(v_{1} v_{2}\right)^{2} \\
& -12\left(n_{12} v_{1}\right)\left(n_{12} v_{2}\right)^{2} v_{2}^{2}+12\left(n_{12} v_{2}\right)^{3} v_{2}^{2}+\left(n_{12} v_{2}\right) v_{1}^{2} v_{2}^{2}-4\left(n_{12} v_{1}\right)\left(v_{1} v_{2}\right) v_{2}^{2} \\
& \left.+8\left(n_{12} v_{2}\right)\left(v_{1} v_{2}\right) v_{2}^{2}+4\left(n_{12} v_{1}\right) v_{2}^{4}-7\left(n_{12} v_{2}\right) v_{2}^{4}\right) \\
& +\frac{G^{2} m_{2}^{2}}{r_{12}^{3}}\left(-2\left(n_{12} v_{1}\right)^{2}\left(n_{12} v_{2}\right)+8\left(n_{12} v_{1}\right)\left(n_{12} v_{2}\right)^{2}\right. \\
& \left.+2\left(n_{12} v_{2}\right)^{3}+2\left(n_{12} v_{1}\right)\left(v_{1} v_{2}\right)+4\left(n_{12} v_{2}\right)\left(v_{1} v_{2}\right)-2\left(n_{12} v_{1}\right) v_{2}^{2}-4\left(n_{12} v_{2}\right) v_{2}^{2}\right)
\end{aligned}
$$




$$
\begin{aligned}
& +\frac{G^{2} m_{1} m_{2}}{r_{12}^{3}}\left(-\frac{243}{4}\left(n_{12} v_{1}\right)^{3}+\frac{565}{4}\left(n_{12} v_{1}\right)^{2}\left(n_{12} v_{2}\right)-\frac{269}{4}\left(n_{12} v_{1}\right)\left(n_{12} v_{2}\right)^{2}\right. \\
& -\frac{95}{12}\left(n_{12} v_{2}\right)^{3}+\frac{207}{8}\left(n_{12} v_{1}\right) v_{1}^{2}-\frac{137}{8}\left(n_{12} v_{2}\right) v_{1}^{2}-36\left(n_{12} v_{1}\right)\left(v_{1} v_{2}\right) \\
& \left.+\frac{27}{4}\left(n_{12} v_{2}\right)\left(v_{1} v_{2}\right)+\frac{81}{8}\left(n_{12} v_{1}\right) v_{2}^{2}+\frac{83}{8}\left(n_{12} v_{2}\right) v_{2}^{2}\right) \\
& +\frac{G^{3} m_{2}^{3}}{r_{12}^{4}}\left(4\left(n_{12} v_{1}\right)+5\left(n_{12} v_{2}\right)\right)+\frac{G^{3} m_{1} m_{2}^{2}}{r_{12}^{4}}\left(-\frac{307}{8}\left(n_{12} v_{1}\right)+\frac{479}{8}\left(n_{12} v_{2}\right)\right. \\
& \left.+\frac{123}{32}\left(\left(n_{12} v_{1}\right)-\left(n_{12} v_{2}\right)\right) \pi^{2}\right)+\frac{G^{3} m_{1}^{2} m_{2}}{r_{12}^{4}}\left(\frac{31397}{420}\left(n_{12} v_{1}\right)-\frac{36227}{420}\left(n_{12} v_{2}\right)\right. \\
& \left.\left.\left.-44\left(\left(n_{12} v_{1}\right)-\left(n_{12} v_{2}\right)\right) \ln \left(\frac{r_{12}}{r_{1}^{\prime}}\right)\right)\right]\left(v_{1}^{i}-v_{2}^{i}\right)\right\}+\mathcal{O}(7) .
\end{aligned}
$$

These equations are in full agreement with the known results valid up to the $2.5 \mathrm{PN}$ order [24 26, 31]. They have the correct perturbative limit given by the geodesics of the Schwarzschild metric at the 3PN order. Most importantly, the equations are invariant under Lorentz transformations (developed to 3PN order); this can be checked using for instance the formulas developed in 448. Finally, as we have seen previously, the equations of motion admit a conserved energy at the 3PN order. The study of the Lagrangian (and Hamiltonian) formulation of these equations is reported in a separate work [37]. The energy is given by

$$
\begin{aligned}
E=\frac{m_{1} v_{1}^{2}}{2}-\frac{G m_{1} m_{2}}{2 r_{12}} & \\
+\frac{1}{c^{2}} & \left\{\frac{G^{2} m_{1}^{2} m_{2}}{2 r_{12}^{2}}+\frac{3 m_{1} v_{1}^{4}}{8}+\frac{G m_{1} m_{2}}{r_{12}}\left(-\frac{1}{4}\left(n_{12} v_{1}\right)\left(n_{12} v_{2}\right)+\frac{3}{2} v_{1}^{2}-\frac{7}{4}\left(v_{1} v_{2}\right)\right)\right\} \\
+\frac{1}{c^{4}} & \left\{-\frac{G^{3} m_{1}^{3} m_{2}}{2 r_{12}^{3}}-\frac{19 G^{3} m_{1}^{2} m_{2}^{2}}{8 r_{12}^{3}}+\frac{5 m_{1} v_{1}^{6}}{16}+\frac{G m_{1} m_{2}}{r_{12}}\left(\frac{3}{8}\left(n_{12} v_{1}\right)^{3}\left(n_{12} v_{2}\right)\right.\right. \\
& +\frac{3}{16}\left(n_{12} v_{1}\right)^{2}\left(n_{12} v_{2}\right)^{2}-\frac{9}{8}\left(n_{12} v_{1}\right)\left(n_{12} v_{2}\right) v_{1}^{2}-\frac{13}{8}\left(n_{12} v_{2}\right)^{2} v_{1}^{2}+\frac{21}{8} v_{1}^{4} \\
+ & \left.\frac{13}{8}\left(n_{12} v_{1}\right)^{2}\left(v_{1} v_{2}\right)+\frac{3}{4}\left(n_{12} v_{1}\right)\left(n_{12} v_{2}\right)\left(v_{1} v_{2}\right)-\frac{55}{8} v_{1}^{2}\left(v_{1} v_{2}\right)+\frac{17}{8}\left(v_{1} v_{2}\right)^{2}+\frac{31}{16} v_{1}^{2} v_{2}^{2}\right) \\
& \left.+\frac{G^{2} m_{1}^{2} m_{2}}{r_{12}^{2}}\left(\frac{29}{4}\left(n_{12} v_{1}\right)^{2}-\frac{13}{4}\left(n_{12} v_{1}\right)\left(n_{12} v_{2}\right)+\frac{1}{2}\left(n_{12} v_{2}\right)^{2}-\frac{3}{2} v_{1}^{2}+\frac{7}{4} v_{2}^{2}\right)\right\} \\
+\frac{1}{c^{6}} & \left\{\frac{35 m_{1} v_{1}^{8}}{128}+\frac{G m_{1} m_{2}}{r_{12}}\left(-\frac{5}{16}\left(n_{12} v_{1}\right)^{5}\left(n_{12} v_{2}\right)-\frac{5}{16}\left(n_{12} v_{1}\right)^{4}\left(n_{12} v_{2}\right)^{2}\right.\right. \\
& -\frac{5}{32}\left(n_{12} v_{1}\right)^{3}\left(n_{12} v_{2}\right)^{3}+\frac{19}{16}\left(n_{12} v_{1}\right)^{3}\left(n_{12} v_{2}\right) v_{1}^{2}+\frac{15}{16}\left(n_{12} v_{1}\right)^{2}\left(n_{12} v_{2}\right)^{2} v_{1}^{2} \\
& +\frac{3}{4}\left(n_{12} v_{1}\right)\left(n_{12} v_{2}\right)^{3} v_{1}^{2}+\frac{19}{16}\left(n_{12} v_{2}\right)^{4} v_{1}^{2}-\frac{21}{16}\left(n_{12} v_{1}\right)\left(n_{12} v_{2}\right) v_{1}^{4}-2\left(n_{12} v_{2}\right)^{2} v_{1}^{4}+\frac{55}{16} v_{1}^{6} \\
& -\frac{19}{16}\left(n_{12} v_{1}\right)^{4}\left(v_{1} v_{2}\right)-\left(n_{12} v_{1}\right)^{3}\left(n_{12} v_{2}\right)\left(v_{1} v_{2}\right)-\frac{15}{32}\left(n_{12} v_{1}\right)^{2}\left(n_{12} v_{2}\right)^{2}\left(v_{1} v_{2}\right)
\end{aligned}
$$




$$
\begin{aligned}
& +\frac{45}{16}\left(n_{12} v_{1}\right)^{2} v_{1}^{2}\left(v_{1} v_{2}\right)+\frac{5}{4}\left(n_{12} v_{1}\right)\left(n_{12} v_{2}\right) v_{1}^{2}\left(v_{1} v_{2}\right)+\frac{11}{4}\left(n_{12} v_{2}\right)^{2} v_{1}^{2}\left(v_{1} v_{2}\right) \\
& -\frac{139}{16} v_{1}^{4}\left(v_{1} v_{2}\right)-\frac{3}{4}\left(n_{12} v_{1}\right)^{2}\left(v_{1} v_{2}\right)^{2}+\frac{5}{16}\left(n_{12} v_{1}\right)\left(n_{12} v_{2}\right)\left(v_{1} v_{2}\right)^{2}+\frac{41}{8} v_{1}^{2}\left(v_{1} v_{2}\right)^{2} \\
& \left.+\frac{1}{16}\left(v_{1} v_{2}\right)^{3}-\frac{45}{16}\left(n_{12} v_{1}\right)^{2} v_{1}^{2} v_{2}^{2}-\frac{23}{32}\left(n_{12} v_{1}\right)\left(n_{12} v_{2}\right) v_{1}^{2} v_{2}^{2}+\frac{79}{16} v_{1}^{4} v_{2}^{2}-\frac{161}{32} v_{1}^{2}\left(v_{1} v_{2}\right) v_{2}^{2}\right) \\
& +\frac{G^{2} m_{1}^{2} m_{2}}{r_{12}^{2}}\left(-\frac{49}{8}\left(n_{12} v_{1}\right)^{4}+\frac{75}{8}\left(n_{12} v_{1}\right)^{3}\left(n_{12} v_{2}\right)-\frac{187}{8}\left(n_{12} v_{1}\right)^{2}\left(n_{12} v_{2}\right)^{2}\right. \\
& +\frac{247}{24}\left(n_{12} v_{1}\right)\left(n_{12} v_{2}\right)^{3}+\frac{49}{8}\left(n_{12} v_{1}\right)^{2} v_{1}^{2}+\frac{81}{8}\left(n_{12} v_{1}\right)\left(n_{12} v_{2}\right) v_{1}^{2}-\frac{21}{4}\left(n_{12} v_{2}\right)^{2} v_{1}^{2}+\frac{11}{2} v_{1}^{4} \\
& -\frac{15}{2}\left(n_{12} v_{1}\right)^{2}\left(v_{1} v_{2}\right)-\frac{3}{2}\left(n_{12} v_{1}\right)\left(n_{12} v_{2}\right)\left(v_{1} v_{2}\right)+\frac{21}{4}\left(n_{12} v_{2}\right)^{2}\left(v_{1} v_{2}\right)-27 v_{1}^{2}\left(v_{1} v_{2}\right) \\
& +\frac{55}{2}\left(v_{1} v_{2}\right)^{2}+\frac{49}{4}\left(n_{12} v_{1}\right)^{2} v_{2}^{2}-\frac{27}{2}\left(n_{12} v_{1}\right)\left(n_{12} v_{2}\right) v_{2}^{2}+\frac{3}{4}\left(n_{12} v_{2}\right)^{2} v_{2}^{2}+\frac{55}{4} v_{1}^{2} v_{2}^{2} \\
& \left.-28\left(v_{1} v_{2}\right) v_{2}^{2}+\frac{135}{16} v_{2}^{4}\right)+\frac{3 G^{4} m_{1}^{4} m_{2}}{8 r_{12}^{4}}+\frac{G^{4} m_{1}^{3} m_{2}^{2}}{r_{12}^{4}}\left(\frac{5809}{280}-\frac{11}{3} \lambda-\frac{22}{3} \ln \left(\frac{r_{12}}{r_{1}^{\prime}}\right)\right) \\
& +\frac{G^{3} m_{1}^{2} m_{2}^{2}}{r_{12}^{3}}\left(\frac{547}{12}\left(n_{12} v_{1}\right)^{2}-\frac{3115}{48}\left(n_{12} v_{1}\right)\left(n_{12} v_{2}\right)-\frac{123}{64}\left(n_{12} v_{1}\right)^{2} \pi^{2}\right. \\
& \left.+\frac{123}{64}\left(n_{12} v_{1}\right)\left(n_{12} v_{2}\right) \pi^{2}-\frac{575}{18} v_{1}^{2}+\frac{41}{64} \pi^{2} v_{1}^{2}+\frac{4429}{144}\left(v_{1} v_{2}\right)-\frac{41}{64} \pi^{2}\left(v_{1} v_{2}\right)\right) \\
& +\frac{G^{3} m_{1}^{3} m_{2}}{r_{12}^{3}}\left(-\frac{44627}{840}\left(n_{12} v_{1}\right)^{2}+\frac{32027}{840}\left(n_{12} v_{1}\right)\left(n_{12} v_{2}\right)+\frac{3}{2}\left(n_{12} v_{2}\right)^{2}+\frac{24187}{2520} v_{1}^{2}\right. \\
& -\frac{27967}{2520}\left(v_{1} v_{2}\right)+\frac{5}{4} v_{2}^{2}+22\left(n_{12} v_{1}\right)^{2} \ln \left(\frac{r_{12}}{r_{1}^{\prime}}\right)-22\left(n_{12} v_{1}\right)\left(n_{12} v_{2}\right) \ln \left(\frac{r_{12}}{r_{1}^{\prime}}\right) \\
& \left.\left.-\frac{22}{3} v_{1}^{2} \ln \left(\frac{r_{12}}{r_{1}^{\prime}}\right)+\frac{22}{3}\left(v_{1} v_{2}\right) \ln \left(\frac{r_{12}}{r_{1}^{\prime}}\right)\right)\right\}+1 \leftrightarrow 2+\mathcal{O}(7) \text {. }
\end{aligned}
$$

This energy is conserved in the sense that its time-derivative computed with the 3PN equations of motion equals the radiation reaction effect at the $2.5 \mathrm{PN}$ order, namely

$$
\begin{aligned}
\frac{d E}{d t}=\frac{4}{5} & \frac{G^{2} m_{1}^{2} m_{2}}{c^{5} r_{12}^{3}}\left[\left(v_{1} v_{12}\right)\left(-v_{12}^{2}+2 \frac{G m_{1}}{r_{12}}-8 \frac{G m_{2}}{r_{12}}\right)\right. \\
& \left.+\left(n_{12} v_{1}\right)\left(n_{12} v_{12}\right)\left(3 v_{12}^{2}-6 \frac{G m_{1}}{r_{12}}+\frac{52}{3} \frac{G m_{2}}{r_{12}}\right)\right]+1 \leftrightarrow 2+\mathcal{O}(7) .
\end{aligned}
$$

The rather complicated expressions (7.16)-(7.17) simplify drastically in the case where the orbit is circular [apart from the gradual inspiral associated with the balance equation (7.18)] and where we place ourselves in the center-of-mass frame. The circular orbit corresponds to the physical situation of the inspiralling compact binaries which motivate our work [1 7 . Here, we give the result concerning circular orbits without proof (see also [34]). The relative acceleration reads

$$
\frac{d \mathbf{v}_{12}}{d t}=-\omega^{2} \mathbf{y}_{12}+\mathbf{F}_{\text {reac }}+\mathcal{O}(7)
$$


where $\mathbf{F}_{\text {reac }}$ is the standard radiation-reaction force in harmonic coordinates,

$$
\mathbf{F}_{\text {reac }}=-\frac{32}{5} \frac{G^{3} m^{3} \nu}{c^{5} r_{12}^{4}} \mathbf{v}_{12}
$$

( $\nu=\frac{m_{1} m_{2}}{m^{2}}$ being the symmetric mass ratio), and where the orbital frequency $\omega$ of the relative circular motion is given to the $3 \mathrm{PN}$ order by

$$
\begin{aligned}
\omega^{2} & =\frac{G m}{r_{12}^{3}}\left\{1+(-3+\nu) \gamma+\left(6+\frac{41}{4} \nu+\nu^{2}\right) \gamma^{2}\right. \\
& \left.+\left(-10+\left[-\frac{67759}{840}+\frac{41}{64} \pi^{2}+22 \ln \left(\frac{r_{12}}{r_{0}^{\prime}}\right)+\frac{44}{3} \lambda\right] \nu+\frac{19}{2} \nu^{2}+\nu^{3}\right) \gamma^{3}+\mathcal{O}(8)\right\} .
\end{aligned}
$$

The post-Newtonian parameter is defined by $\gamma=\frac{G m}{r_{12} c^{2}}$, and we recall that $r_{12}=\left|\mathbf{y}_{1}-\mathbf{y}_{2}\right|$ is the orbital separation in harmonic coordinates. The constant $r_{0}^{\prime}$ appearing in the logarithm is related to the two constants $r_{1}^{\prime}$ and $r_{2}^{\prime}$ by

$$
\ln r_{0}^{\prime}=\frac{m_{1}}{m} \ln r_{1}^{\prime}+\frac{m_{2}}{m} \ln r_{2}^{\prime} .
$$

The $3 \mathrm{PN}$ energy $E$ in the center of mass of the particles, which is such that $\frac{d E}{d t}=0$ as a consequence of the conservative equations of motion, is obtained as

$$
\begin{aligned}
E & =-\frac{1}{2} \mu c^{2} \gamma\left\{1+\left(-\frac{7}{4}+\frac{1}{4} \nu\right) \gamma+\left(-\frac{7}{8}+\frac{49}{8} \nu+\frac{1}{8} \nu^{2}\right) \gamma^{2}\right. \\
& \left.+\left(-\frac{235}{64}+\left[\frac{106301}{6720}-\frac{123}{64} \pi^{2}+\frac{22}{3} \ln \left(\frac{r_{12}}{r_{0}^{\prime}}\right)-\frac{22}{3} \lambda\right] \nu+\frac{27}{32} \nu^{2}+\frac{5}{64} \nu^{3}\right) \gamma^{3}+\mathcal{O}(8)\right\} .
\end{aligned}
$$

The invariant 3PN energy follows from the replacement of the post-Newtonian parameter $\gamma$ by its expression in terms of the frequency $\omega$ as deduced from computing the inverse of (7.21). We find

$$
\begin{aligned}
E & =-\frac{1}{2} \mu c^{2} x\left\{1+\left(-\frac{3}{4}-\frac{1}{12} \nu\right) x+\left(-\frac{27}{8}+\frac{19}{8} \nu-\frac{1}{24} \nu^{2}\right) x^{2}\right. \\
& \left.+\left(-\frac{675}{64}+\left[\frac{209323}{4032}-\frac{205}{96} \pi^{2}-\frac{110}{9} \lambda\right] \nu-\frac{155}{96} \nu^{2}-\frac{35}{5184} \nu^{3}\right) x^{3}+\mathcal{O}(8)\right\},
\end{aligned}
$$

where the parameter $x$ is defined by

$$
x=\left(\frac{G m \omega}{c^{3}}\right)^{2 / 3} .
$$


Note that the logarithm disappeared from the invariant expression of the energy (7.24), in agreement with the fact that it is pure gauge. However, the constant $\lambda$ stays in the final formula; the static ambiguity constant $\omega_{s}$ of Jaranowski and Schäfer [33] is related to it by $\omega_{s}=-\frac{11}{3} \lambda-\frac{1987}{840}($ see 34$)$.

\section{ACKNOWLEDGMENTS}

Most of the algebraic manipulations reported in this article have been done with the help of the softwares Mathematica and MathTensor. One of us (G.F.) would like to acknowledge Jean-Marc Conan and Vincent Michau for letting him complete this work in the context of his military service.

\section{APPENDIX A: SUM OF LEIBNIZ TERMS}

In this appendix we give the sum of all the terms of the type $\delta_{\text {Leibniz }} T$ introduced by (3.23) that we have encountered during the process of simplification of the $3 \mathrm{PN}$ potentials. The reduction of these terms using the distributional derivative is done in Section VI.

$$
\begin{aligned}
\delta_{\text {Leibniz }} & \left(\frac{h^{00}+h^{i i}}{2}\right)=\square_{\mathcal{R}}^{-1}\left\{-\frac{8}{c^{4}}\left(\partial_{i} \operatorname{Pf} V \partial_{i} \operatorname{Pf} V+V \square \operatorname{Pf} V-\frac{1}{2} \square\left(\operatorname{Pf} V^{2}\right)-\frac{1}{c^{2}}\left(\partial_{t} \operatorname{Pf} V\right)^{2}\right)\right. \\
- & \frac{8}{c^{6}}\left(\partial_{i} \operatorname{Pf} V \partial_{i} \operatorname{Pf} \hat{W}+\frac{1}{2} V \square \operatorname{Pf} \hat{W}+\frac{1}{2} \hat{W} \square \operatorname{Pf} V-\frac{1}{2} \square(\operatorname{Pf} V \hat{W})-\frac{1}{c^{2}} \partial_{t} \operatorname{Pf} V \partial_{t} \operatorname{Pf} \hat{W}\right) \\
- & \frac{32}{c^{6}}\left(V \partial_{i} \operatorname{Pf} V \partial_{i} \operatorname{Pf} V+\frac{1}{2} V^{2} \square \operatorname{Pf} V-\frac{1}{c^{2}} V\left(\partial_{t} \operatorname{Pf} V\right)^{2}-\frac{1}{6} \square\left(\operatorname{Pf} V^{3}\right)\right) \\
- & \frac{32}{c^{6}}\left(\partial_{i} \operatorname{Pf} V \partial_{i}\left(\operatorname{Pf} V^{2}\right)-2 V \partial_{i} \operatorname{Pf} V \partial_{i} \operatorname{Pf} V\right) \\
- & \frac{16}{c^{8}}\left(V \partial_{t}^{2}\left(\operatorname{Pf} V^{2}\right)-2 V\left(\partial_{t} \operatorname{Pf} V\right)^{2}-2 V^{2} \partial_{t}^{2} \operatorname{Pf} V\right) \\
- & \frac{16}{c^{8}}\left(\partial_{t} \operatorname{Pf} V \partial_{t}\left(\operatorname{Pf} V^{2}\right)-2 V\left(\partial_{t} \operatorname{Pf} V\right)^{2}\right) \\
- & \frac{32}{c^{8}}\left(V_{i} \partial_{t} \partial_{i}\left(\operatorname{Pf} V^{2}\right)-2 V V_{i} \partial_{t} \partial_{i} \operatorname{Pf} V-2 V_{i} \partial_{t} \operatorname{Pf} V \partial_{i} \operatorname{Pf} V\right) \\
- & \frac{64}{c^{8}}\left(V^{2} \partial_{i} \operatorname{Pf} V \partial_{i} \operatorname{Pf} V+\frac{1}{3} V^{3} \square \operatorname{Pf} V-\frac{1}{12} \square\left(\operatorname{Pf} V^{4}\right)\right) \\
- & \frac{32}{c^{8}}\left(\partial_{i}\left(\operatorname{Pf} V^{2}\right) \partial_{i}\left(\operatorname{Pf} V^{2}\right)-4 V^{2} \partial_{i} \operatorname{Pf} V \partial_{i} \operatorname{Pf} V\right) \\
+ & \frac{144}{c^{8}}\left(V \partial_{i} \operatorname{Pf} V \partial_{i}\left(\operatorname{Pf} V^{2}\right)-2 V^{2} \partial_{i} \operatorname{Pf} V \partial_{i} \operatorname{Pf} V\right) \\
- & \frac{128}{3 c^{8}}\left(\partial_{i} \operatorname{Pf} V \partial_{i}\left(\operatorname{Pf} V^{3}\right)-3 V^{2} \partial_{i} \operatorname{Pf} V \partial_{i} \operatorname{Pf} V\right)
\end{aligned}
$$


$+\frac{64}{c^{8}}\left(\partial_{i} \operatorname{Pf} V_{j} \partial_{j}\left(\operatorname{Pf} V V_{i}\right)-V \partial_{i} \operatorname{Pf} V_{j} \partial_{j} \operatorname{Pf} V_{i}-V_{i} \partial_{i} \operatorname{Pf} V_{j} \partial_{j} \operatorname{Pf} V\right)$

$-\frac{16}{c^{8}}\left(\hat{W}_{i j} \partial_{i j}\left(\operatorname{Pf} V^{2}\right)-2 V \hat{W}_{i j} \partial_{i j} \operatorname{Pf} V-2 \hat{W}_{i j} \partial_{i} \operatorname{Pf} V \partial_{j} \operatorname{Pf} V\right)$

$-\frac{4}{c^{8}}\left(\partial_{i} \operatorname{Pf} \hat{W} \partial_{i} \operatorname{Pf} \hat{W}+\hat{W} \square \operatorname{Pf} \hat{W}-\frac{1}{2} \square\left(\operatorname{Pf} \hat{W}^{2}\right)\right)$

$-\frac{16}{c^{8}}\left(\hat{W} \partial_{i} \operatorname{Pf} V \partial_{i} \operatorname{Pf} V+2 V \partial_{i} \operatorname{Pf} V \partial_{i} \operatorname{Pf} \hat{W}+V \hat{W} \square \operatorname{Pf} V+\frac{1}{2} V^{2} \square \operatorname{Pf} \hat{W}-\frac{1}{2} \square\left(\operatorname{Pf} V^{2} \hat{W}\right)\right)$

$+\frac{8}{c^{8}}\left(\hat{W} \square\left(\operatorname{Pf} V^{2}\right)-2 V \hat{W} \square \operatorname{Pf} V-2 \hat{W} \partial_{i} \operatorname{Pf} V \partial_{i} \operatorname{Pf} V\right)$

$-\frac{16}{c^{8}}\left(\partial_{i} \operatorname{Pf} \hat{W} \partial_{i}\left(\operatorname{Pf} V^{2}\right)-2 V \partial_{i} \operatorname{Pf} \hat{W} \partial_{i} \operatorname{Pf} V\right)$

$-\frac{32}{c^{8}}\left(\partial_{i} \operatorname{Pf} V \partial_{i}(\operatorname{Pf} V \hat{W})-V \partial_{i} \operatorname{Pf} V \partial_{i} \operatorname{Pf} \hat{W}-\hat{W} \partial_{i} \operatorname{Pf} V \partial_{i} \operatorname{Pf} V\right)$

$+\frac{8}{c^{8}}\left(\partial_{k} \operatorname{Pf} \hat{W}_{i j} \partial_{k} \operatorname{Pf} \hat{W}_{i j}+\hat{W}_{i j} \square \operatorname{Pf} \hat{W}_{i j}-\frac{1}{2} \square\left(\operatorname{Pf} \hat{W}_{i j} \hat{W}_{i j}\right)\right)$

$-\frac{64}{c^{8}}\left(\partial_{i} \operatorname{Pf} V \partial_{i} \operatorname{Pf}\left(\hat{X}+\frac{1}{2} \hat{Z}\right)+\frac{1}{2} V \square \operatorname{Pf}\left(\hat{X}+\frac{1}{2} \hat{Z}\right)+\frac{1}{2}\left(\hat{X}+\frac{1}{2} \hat{Z}\right) \square \operatorname{Pf} V\right.$

$\left.\left.-\frac{1}{2} \square\left(\operatorname{Pf} V\left(\hat{X}+\frac{1}{2} \hat{Z}\right)\right)\right)\right\}+\mathcal{O}(10)$,

$\delta_{\text {Leibniz }} h^{0 i}=$

$\square_{\mathcal{R}}^{-1}\left\{-\frac{16}{c^{5}}\left(\partial_{j} \operatorname{Pf} V \partial_{j} \operatorname{Pf} V_{i}+\frac{1}{2} V \square \operatorname{Pf} V_{i}+\frac{1}{2} V_{i} \square \operatorname{Pf} V-\frac{1}{2} \square\left(\operatorname{Pf} V V_{i}\right)-\frac{1}{c^{2}} \partial_{t} \operatorname{Pf} V \partial_{t} \operatorname{Pf} V_{i}\right)\right.$

$+\frac{24}{c^{7}}\left(\partial_{t} \operatorname{Pf} V \partial_{i}\left(\operatorname{Pf} V^{2}\right)-2 V \partial_{t} \operatorname{Pf} V \partial_{i} \operatorname{Pf} V\right)$

$+\frac{24}{c^{7}}\left(\partial_{i} \operatorname{Pf} V \partial_{t}\left(\operatorname{Pf} V^{2}\right)-2 V \partial_{i} \operatorname{Pf} V \partial_{t} \operatorname{Pf} V\right)$

$-\frac{32}{c^{7}}\left(\partial_{j} \operatorname{Pf} V \partial_{j} \operatorname{Pf} \hat{R}_{i}+\frac{1}{2} V \square \operatorname{Pf} \hat{R}_{i}+\frac{1}{2} \hat{R}_{i} \square \operatorname{Pf} V-\frac{1}{2} \square\left(\operatorname{Pf} V \hat{R}_{i}\right)\right)$

$-\frac{8}{c^{7}}\left(\partial_{j} \operatorname{Pf} V_{i} \partial_{j} \operatorname{Pf} \hat{W}+\frac{1}{2} V_{i} \square \operatorname{Pf} \hat{W}+\frac{1}{2} \hat{W} \square \operatorname{Pf} V_{i}-\frac{1}{2} \square\left(\operatorname{Pf} V_{i} \hat{W}\right)\right)$

$+\frac{32}{c^{8}}\left(\partial_{j} \operatorname{Pf} V \partial_{i}\left(\operatorname{Pf} V V_{j}\right)-V \partial_{j} \operatorname{Pf} V \partial_{i} \operatorname{Pf} V_{j}-V_{j} \partial_{j} \operatorname{Pf} V \partial_{i} \operatorname{Pf} V\right)$

$-\frac{32}{c^{8}}\left(\partial_{j} \operatorname{Pf} V \partial_{j}\left(\operatorname{Pf} V V_{i}\right)-V \partial_{j} \operatorname{Pf} V \partial_{j} \operatorname{Pf} V_{i}-V_{i} \partial_{j} \operatorname{Pf} V \partial_{j} \operatorname{Pf} V\right)$

$+\frac{32}{c^{7}}\left(\partial_{i} \operatorname{Pf} V_{j} \partial_{j}\left(\operatorname{Pf} V^{2}\right)-2 V \partial_{i} \operatorname{Pf} V_{j} \partial_{j} \operatorname{Pf} V\right)$

$-\frac{32}{c^{7}}\left(\partial_{j} \operatorname{Pf} V_{i} \partial_{j}\left(\operatorname{Pf} V^{2}\right)-2 V \partial_{j} \operatorname{Pf} V_{i} \partial_{j} \operatorname{Pf} V\right)$ 


$$
\begin{aligned}
&+\frac{16}{c^{7}}\left(\partial_{k} \operatorname{Pf} V_{j} \partial_{k} \operatorname{Pf} \hat{W}_{i j}+\frac{1}{2} V_{j} \square \operatorname{Pf} \hat{W}_{i j}+\frac{1}{2} \hat{W}_{i j} \square \operatorname{Pf} V_{j}-\frac{1}{2} \square\left(\operatorname{Pf} V_{j} \hat{W}_{i j}\right)\right) \\
&\left.-\frac{32}{c^{7}}\left(V_{i} \partial_{j} \operatorname{Pf} V \partial_{j} \operatorname{Pf} V+2 V \partial_{j} \operatorname{Pf} V \partial_{j} \operatorname{Pf} V_{i}+\frac{1}{2} V^{2} \square \operatorname{Pf} V_{i}+V V_{i} \square \operatorname{Pf} V-\frac{1}{2} \square\left(\operatorname{Pf} V^{2} V_{i}\right)\right)\right\} \\
&+\mathcal{O}(9), \\
& \delta_{\text {Leibniz }} h^{i j}= \text { (A2) } \\
& \square_{\mathcal{R}}^{-1}\left\{\frac{16}{c^{6}}\left(\partial_{(i} \operatorname{Pf} V \partial_{j)}\left(\operatorname{Pf} V^{2}\right)-2 V \partial_{(i} \operatorname{Pf} V \partial_{j} \operatorname{Pf} V\right)\right. \\
&\left.-\frac{8}{c^{6}} \delta_{i j}\left(\partial_{k} \operatorname{Pf} V \partial_{k}\left(\operatorname{Pf} V^{2}\right)-2 V \partial_{k} \operatorname{Pf} V \partial_{k} \operatorname{Pf} V\right)\right\}+\mathcal{O}(8) .
\end{aligned}
$$




\section{REFERENCES}

[1] C. Cutler, T.A. Apostolatos, L. Bildsten, L.S. Finn, E.E. Flanagan, D. Kennefick, D.M. Markovic, A. Ori, E. Poisson, G.J. Sussman and K.S. Thorne, Phys. Rev. Lett. 70, 2984 (1993).

[2] C. Cutler, L.S. Finn, E. Poisson and G.J. Sussman, Phys. Rev. D47, 1511 (1993).

[3] L.S. Finn and D.F. Chernoff, Phys. Rev. D47, 2198 (1993).

[4] C. Cutler and E.E. Flanagan, Phys. Rev. D49, 2658 (1994).

[5] H. Tagoshi and T. Nakamura, Phys. Rev. D49, 4016 (1994).

[6] E. Poisson, Phys. Rev. D52, 5719 (1995).

[7] T. Damour, B.R. Iyer and B.S. Sathyaprakash, Phys. Rev. D57, 885 (1998).

[8] H.A. Lorentz and J.Droste, Versl. K. Akad. Wet. Amsterdam 26, 392 and 649 (1917); in the collected papers of H.A. Lorentz, vol. 5, The Hague, Nijhoff (1937).

[9] T. Damour, in Gravitational Radiation, N. Deruelle and T. Piran (eds.), North-Holland Company (1983).

[10] T. Damour, in 300 years of Gravitation, S. W. Hawking and W. Israel (eds.), Cambridge U. Press (1987).

[11] A. Einstein, L. Infeld and B. Hoffmann, Ann. Math. 39, 65 (1938).

[12] A. Einstein and L. Infeld, Ann. Math. 41, 797 (1940).

[13] A. Einstein and L. Infeld, Can. J. Math. 1, 209 (1949).

[14] Y. Itoh, T. Futamase and H. Asada, gr-qc 9910052.

[15] V. Fock, J. Phys. (U.S.S.R.) 1, 81 (1939).

[16] N. Petrova, J. Phys. (U.S.S.R.) 19, 989 (1949).

[17] A. Papapetrou, Proc. Phys. Soc. (London) 64, 57 (1951).

[18] T. Ohta, H. Okamura, T. Kimura and K. Hiida, Progr. Theor. Phys. 50, 492 (1973).

[19] T. Ohta, H. Okamura, T. Kimura and K. Hiida, Progr. Theor. Phys. 51, 1220 (1974).

[20] T. Ohta, H. Okamura, T. Kimura and K. Hiida, Progr. Theor. Phys. 51, 1598 (1974).

[21] T. Damour and G. Schäfer, Gen. Rel. Grav. 17, 879 (1985).

[22] G. Schäfer, Phys. Lett. A123, 336 (1987).

[23] L. Bel, T. Damour, N. Deruelle, J. Ibañez and J. Martin, Gen. Relativ. Gravit. 13, 963 (1981). 
[24] T. Damour and N. Deruelle, Phys. Lett. 87A, 81 (1981).

[25] N. Deruelle, thèse de doctorat d'état, Paris (1982).

[26] T. Damour, C. R. Acad. Sc. Paris, 294, 1355 (1982).

[27] S.M. Kopejkin, Astron. Zh. 62, 889 (1985).

[28] L.P. Grishchuk and S.M. Kopejkin, in Relativity in Celestial Mechanics and Astrometry, J. Kovalevsky and V.A. Brumberg (eds.), Reidel, Dordrecht (1986).

[29] G. Schäfer, Ann. Phys. (N.Y.) 161, 81 (1985).

[30] G. Schäfer, Gen. Rel. Grav. 18, 255 (1986).

[31] L. Blanchet, G. Faye and B. Ponsot, Phys. Rev. D58, 124002 (1998).

[32] P. Jaranowski and G. Schäfer, Phys. Rev. D57, 7274 (1998).

[33] P. Jaranowski and G. Schäfer, Phys. Rev. D60, 124003 (1999).

[34] L. Blanchet and G. Faye, Phys. Lett. A271, 58 (2000).

[35] T. Damour, P. Jaranowski and G. Schäfer, Phys. Rev. D62, 021501(R) (2000).

[36] T. Damour, P. Jaranowski and G. Schäfer, gr-qc 0010040.

[37] V.C. de Andrade, L. Blanchet and G. Faye, submitted to Class. Quantum Grav., gr-qd 0011063 .

[38] S. Chandrasekhar and Y. Nutku, Astrophys. J. 158, 55 (1969).

[39] S. Chandrasekhar and F.P. Esposito, Astrophys. J. 160, 153 (1970).

[40] J. Ehlers, Ann. N.Y. Acad. Sci. 336, 279 (1980).

[41] G.D. Kerlick, Gen. Rel. Grav. 12, 467 (1980).

[42] G.D. Kerlick, Gen. Rel. Grav. 12, 521 (1980).

[43] A. Papapetrou and B. Linet, Gen. Rel. Grav. 13, 335 (1981).

[44] J. Hadamard, Le problème de Cauchy et les équations aux dérivées partielles linéaires hyperboliques, Paris: Hermann (1932).

[45] L. Schwartz, Théorie des distributions, Paris: Hermann (1978).

[46] A. Sellier, Proc. R. Soc. Lond. A445, 69 (1964).

[47] L. Blanchet and G. Faye, J. Math. Phys. 41, 7675 (2000).

[48] L. Blanchet and G. Faye, submitted to J. Math. Phys., gr-qc 0006100.

[49] L. Infeld, Rev. Mod. Phys. 29, 398 (1957). 
[50] L. Infeld and J. Plebanski, Motion and Relativity, Pergamon, London (1960).

[51] M. Riesz, Acta Mathematica 81, 1 (1949).

[52] G. Faye, Thèse de docteur en Physique Théorique, Université Paris VI, unpublished (1999).

[53] V.A. Fock, Theory of Space, Time and Gravitation, Pergamon, London (1959).

[54] L. Blanchet and T. Damour, Philos. Trans. R. Soc. Lond. A320, 379 (1986).

[55] L. Blanchet, Class. Quantum Grav. 15, 1971 (1998).

[56] L. Blanchet, Phys. Rev. D47, 4392 (1993).

[57] L. Blanchet, T. Damour and B.R. Iyer, Phys. Rev. D51, 5360 (1995).

[58] T. Damour and B.R. Iyer, Ann. Inst. H. Poincaré (Phys. Théorique) 54, 115 (1991).

[59] T. Damour and G. Esposito-Farèse, Phys. Rev. D53, 5541 (1996).

[60] L. Schwartz, C. R. Acad Sc. Paris 239, 847 (1954). 